\title{
An Examination of the Buffering Effect of Positive Emotions on Cardiovascular Reactivity and Recovery
}

\author{
Casey E. Cavanagh
}

Follow this and additional works at: https://researchrepository.wvu.edu/etd

\section{Recommended Citation}

Cavanagh, Casey E., "An Examination of the Buffering Effect of Positive Emotions on Cardiovascular Reactivity and Recovery" (2016). Graduate Theses, Dissertations, and Problem Reports. 5327. https://researchrepository.wvu.edu/etd/5327

This Dissertation is protected by copyright and/or related rights. It has been brought to you by the The Research Repository @ WVU with permission from the rights-holder(s). You are free to use this Dissertation in any way that is permitted by the copyright and related rights legislation that applies to your use. For other uses you must obtain permission from the rights-holder(s) directly, unless additional rights are indicated by a Creative Commons license in the record and/ or on the work itself. This Dissertation has been accepted for inclusion in WVU Graduate Theses, Dissertations, and Problem Reports collection by an authorized administrator of The Research Repository @ WVU. For more information, please contact researchrepository@mail.wvu.edu. 
An Examination of the Buffering Effect of Positive Emotions on Cardiovascular Reactivity and

$$
\text { Recovery }
$$

Casey E. Cavanagh

Dissertation submitted to the Eberly College of Arts and Sciences at West Virginia University in partial fulfillment of the requirements for the degree of

Doctor of Philosophy

in

Psychology

\author{
Kevin Larkin, Ph.D. Chair \\ Barry Edelstein, Ph.D. \\ Julie Hicks Patrick, Ph.D. \\ Amy Gentzler, Ph.D. \\ Julie Brefczynski-Lewis, Ph.D. \\ Department of Psychology \\ Morgantown, West Virginia \\ 2016
}

Keywords: positive emotions, undoing hypothesis, facial feedback hypothesis, cardiovascular

recovery

Copyright 2016 Casey E. Cavanagh 


\begin{abstract}
An Examination of the Buffering Effect of Positive Emotions on Cardiovascular Reactivity and

Recovery

Casey E. Cavanagh

Positive emotions are related to improved physical and mental health. One potential mechanism through which positive emotions affect physical health is by reducing cardiovascular reactivity to stress and enhancing recovery from stress. The undoing hypothesis proposes physiological recovery from stressful events can be improved by induction of positive emotions. Although there is some research supporting the undoing hypothesis, the evidence is largely mixed. The purpose of the present study was to compare two methods of inducing positive emotions to determine if one method was superior at inducing positive emotions and thereby determine how each method affected cardiovascular recovery from stress among a sample of undergraduate students. The study employed a $2 \times 2$ between-subjects design. The first between subjects factor, Smile, involved a manipulation based on the facial feedback hypothesis (Smile, No-smile). The second between subjects factor, Event, involved exposure to photographs of a pleasant or neutral event (Happy, Neutral). Participants completed a mental arithmetic stressor task while engaging in the positive emotion induction tasks. Measures of cardiovascular responses were obtained and areas under the curve were calculated for each cardiovascular measure during a recovery period following exposure to stress.

Results demonstrated that the smile manipulation was associated with increased diastolic blood pressure responses to stress and that exposure to personally relevant photographs of pleasant events was associated with increased systolic blood pressure responses to stress. No significant effects of positive emotions were observed on measures of cardiovascular recovery from stress.

The findings observed in the current study did not support the undoing hypothesis. There are several possible explanations for the inconsistent findings. The types of positive emotion induction tasks used in the present study differed from previous research, which may account for the differences in findings. Further, recovery in the present study was measured by area under the curve. This method has not previously been used to examine recovery with the undoing hypothesis. Future research should continue to examine the undoing hypothesis to uncover reasons for the lack of consistent findings across studies that have employed various methods of measuring recovery.
\end{abstract}




\section{Acknowledgements}

I would like to thank my committee for their insight and guidance on this project. Thank you especially to Kevin Larkin for his invaluable mentorship, which has helped me develop as a psychologist and researcher. Thank you also to Alex Cheslock and Elizabeth Lee, undergraduate research assistants, for their time and effort in completing this project, which I greatly appreciated. Finally, I would like to thank my parents for their continuous encouragement and support throughout my education. 
Table of Contents

I. Introduction (pp. 1-19)

a. Emotions and Health (pp. 2-4)

b. Cardiovascular Reactivity and Recovery (pp. 4-7)

c. The Undoing Effect of Positive Emotions (pp. 7-10)

d. The Facial Feedback Hypothesis (pp. 11-13)

e. Purpose of the Current Study and Specific Aims (pp. 14-19)

II. Method (pp. 19-30)

a. Participants and Sample Size (pp. 19-21)

i. SONA Screening Phase (pp. 20-21)

ii. Laboratory Phase (p. 21)

b. Experimental Design (p. 21)

c. Measures (pp. 21-24)

i. Demographic Form (p. 21)

ii. Positive and Negative Affect Schedule (pp. 22-23)

iii. Mental Arithmetic Questionnaire (pp. 23-24)

iv. Post-Experiment Questionnaire (pp. 24)

d. Behavioral Observation (pp. 24-25)

e. Apparatus (pp. 25-26)

i. Heart Rate (p. 25)

ii. Heart Rate Variability (p. 25)

iii. Blood Pressure (p. 26)

f. Experimental Stimuli (pp. 26-29) 
i. Stressor Task (pp. 26-27)

ii. Duchenne-Smile Manipulation (pp. 27-28)

iii. Photographs (pp. 28-29)

g. Procedure (pp. 29-30)

i. Screening Phase (p. 29)

ii. Laboratory Phase (pp. 29-30)

III. Results (pp. 30-51)

a. Data Reduction and Assumption Checks (pp. 30-31)

i. Missing Data and Removal of Invalid Blood Pressure Recordings (p. 31)

ii. Resting Physiological Parameters (pp. 31-33)

iii. Physiological Measures during Stress (pp. 33-34)

iv. Physiological Measures during Recovery from Stress (pp. 34-35)

v. Heart Rate (p. 35)

vi. Heart Rate Variability (pp. 35-36)

vii. Testing statistical assumptions (pp. 36-40)

viii. Correlations among Dependent Variables and Covariates (pp. 40-41)

ix. Behavioral Coding (pp. 41-42)

x. Performance on Math Problems (pp. 42-43)

xi. Reactivity to Math Problems (pp. 43-44)

b. Primary Study Analyses on Cardiovascular Parameters (pp. 44-51)

i. Initial Resting Period (pp. 44-45)

ii. Reactivity to the Task (p. 45)

iii. Recovery from the Task (p. 46) 
iv. Examination of Recovery as a Return to Baseline (pp. 46-47)

v. Measures of Affect (p. 47)

vi. Post-Experiment Questionnaire Ratings (pp. 47-48)

1. Difficulty of Manipulation (p. 48)

2. Tiredness of Manipulation (p. 48)

3. Uncomfortableness of Manipulation (p. 48)

4. Distraction (pp. 48-49)

5. Pleasant Thoughts (p. 49)

6. Thinking about Plans after the Study (pp. 49-50)

7. Anticipating a Long Task (p. 50)

8. Ratings of Pleasant Event Photos (p. 50)

9. Ratings of Neutral Photos (p. 51)

10. Purpose of the Study (p. 51)

IV. Discussion (pp. 51-70)

a. Strengths and Limitations (pp. 62-67)

b. Future Directions and Conclusions (pp. 67-70)

V. References (pp. 71-82)

VI. Table 1: Demographic characteristics of the SONA (screening sample, pilot sample, and the lab sample) (p. 83)

VII. Table 2: Additional Demographic characteristics of SONA (screening) sample (pp. 8485)

VIII. Table 3: Means and Standard Deviations for Study Variables (pp. 86-87) 
IX. Table 4: Univariate Correlations between Demographic, Cardiovascular, and SelfReported Affect Variables (pp. 88-89)

X. Table 5: Preliminary Analyses of SBP, DBP, and HR during each minute of the Resting Period (pp. 90-91)

XI. Table 6: Preliminary Analyses of SBP, DBP, and HR during each minute of the Stressor Period (p. 92)

XII. Table 7: Smile by Event ANOVAs on Math Performance and Affinity (pp. 93-95)

XIII. Table 8: Reactivity to the Math Task for each dependent variable (pp. 96-97)

XIV. Table 9: Means, Standard Deviations, and ANOVA Summary Tables for SBP and DPB during the Resting Period (p. 98)

XV. Table 10: Simple Main Effects of DBP for the Smile by Event Interaction during the Resting Period (p. 99)

XVI. Table 11: Means, Standard Deviations, and ANOVA Summary Tables for SBP and DBP during the Stressor Period (p. 100)

XVII. Table 12: Means, Standard Deviations, and ANOVA Summary Tables for SBP and DPB Areas Unver the Curve during the Recovery Period of Blood Pressure Controlling for Law of Initial Values and Reactivity (p. 101)

XVIII. Table 13: Means, Standard Deviations, and ANOVA Summary Tables for Time to Recovery to Initial Resting SBP and DBP (p. 102)

XIX. Table 14: Means, Standard Deviations, and ANOVA Summary Tables for NA and PA following Resting, Stressor, and Recovery Periods (pp. 103-105)

XX. Table 15: Means, Standard Deviations, and ANOVA Summary Tables on Items from the Post-Experiment Questionnaire (pp. 106-107) 
XXI. Table 16: Means, Standard Deviations, and ANOVA Summary Tables on Items Assessing Nature of Distracting Thoughts during the Task from the Post-Experiment Questionnaire (pp. 108-111)

XXII. Table 17: Simple Main Effects for Smile by Event Instructions on Items 6 and 8 on the Post-Experiment Questionnaire (p. 112)

XXIII. Table 18: Ratings of Positive and Neutral Photographs on the Post-Experiment Questionnaire (p. 113)

XXIV. Table 19: Group Differences in Knowledge of Smile Effect from the Post-Experiment Questionnaire (p. 114)

XXV. Appendix A: Pilot Study (pp. 115-116)

XXVI. Appendix B: Demographics Questionnaire (pp. 117-122)

XXVII. Appendix C: Mental Arithmetic Questionnaire (p. 123)

XXVIII. Appendix D: Arithmetic Calculations (p. 124)

XXIX. Appendix E: Smile and No-Smile Manipulation Positioning and Instructions (pp. 125126)

XXX. Appendix F: IAPS (p. 127)

XXXI. Appendix G: Event Instructions (p. 128)

XXXII. Appendix H: Description of the Study (p. 129)

XXXIII. Appendix I: Debriefing (p. 130)

XXXIV. Appendix J: Post-Experiment Questionnaire-A (pp. 131-135)

XXXV. Appendix K: Post-Experiment Questionnaire-B (pp. 136-139) 
An Examination of the Buffering Effect of Positive Emotions on Cardiovascular Reactivity and

\section{Recovery}

Cardiovascular disease (CVD) includes diseases affecting the heart, brain, and blood vessels and is a global health concern. CVD is the leading cause of death worldwide, with more than 17.5 million deaths attributed to cardiovascular diseases in 2012 (World Health Organization, 2015). There are a number of factors that increase the risk of developing CVD, including demographic, genetic, behavioral, and psychological risk factors. Demographic risk factors include, age, gender, ethnicity, and low socioeconomic status (SES) (World Health Organization, 2011; Yusuf, Reddy, Ounpuu, \& Anand, 2001a; 2001b). As people age, our risk for developing CVD increases. In addition, men are at an increased risk of developing CVD compared to premenopausal women. However, after menopause, the risk of developing CVD is relatively similar for men and women (World Health Organization, 2011). In regards to ethnicity, African Americans and Hispanics have an elevated risk profile for developing CVD in comparison to Caucasians (Yusuf et al., 2001a; Yusuf et al., 2001b). Behavioral risk factors are generally modifiable and include tobacco use, consumption of excessive amounts of alcohol, unhealthy diet, and low physical activity (World Health Organization, 2011). It is important to note that demographic and behavioral risk factors are strongly associated. For example, individuals living in low SES areas have limited opportunities to engage in healthy eating in comparison to those living in high SES areas (World Health Organization, 2011). Genetic risk factors for developing CVD include a family history of CVD, high cholesterol, hypertension, and/or diabetes (Yusuf et al., 2001a).

Similar to behavioral risk factors, psychological risk factors such as, stress, depression, and anxiety represent modifiable risk factors and are characterized by a general pattern of 
negative affect (World Health Organization, 2011). The presence of these psychological risk factors highlights the importance of emotional factors and health outcomes with respect to the etiology of CVD.

\section{Emotions and Health}

For a number of years, research has focused exclusively on the detrimental effects of negative affect and emotions on physical health, including CVD. Negative affect (NA) is a superordinate construct of subjective distress and consists of a number of aversive mood states (e.g., anger, disgust, depression, anxiety, fear, etc.) (Watson \& Pennebaker, 1989). Overall, NA is associated with poor physical health and increased risk for CVD. For example, there is strong evidence that NA is associated with poorer outcomes among patients with coronary heart disease (CHD) (DeSteno, Gross, \& Kubzansky, 2013; Kubzansky \& Kawachi, 2000). Anxiety and depression individually have also been identified as risk factors for the development of CHD (Roest, Martens, de Jonge, \& Denollet, 2010; Wulsin, 2003). Further, anxiety is a risk factor for cardiac mortality and depression is predictive of prognosis in patients with CHD (Roest et al., 2010; van Melle et al., 2004). Considering the evidence demonstrating a relation between NA and CVD and the fact that a considerable proportion of the variance in predicting CVD is still unexplained, research in recent years has shifted its focus to examine the effects of positive affect (PA) and positive emotions on physical health outcomes (Boehm \& Kubzansky, 2012; Gallo, Ghaed, \& Bracken, 2004).

Research has demonstrated that PA, which involves the experience of pleasurable feelings (e.g., happiness, joy, excitement, enthusiasm, and contentment) (Watson \& Pennebaker, 1989), is associated with better physical health and may ameliorate the effects of NA. In regards to broad general health, PA predicts improved mental and physical quality of life (Stauber et al., 
2013). In addition, there is preliminary, but strong evidence that PA is associated with a reduction in risk of developing CVD (Boehm \& Kubzansky, 2012). Further, PA has been shown to be protective against CVD as well as other medical problems, including the common cold (Boehm \& Kubzansky, 2012; Cohen, Doyle, Turner, Alper, \& Skoner, 2003). The current evidence suggests that PA influences health broadly and cardiovascular health specifically; however, mechanisms underlying this association are largely unknown.

There are multiple proposed mechanisms that may explain how PA affects cardiovascular health. Positive emotions may promote better decision-making by encouraging less risky (e.g., no smoking) and healthier decisions (e.g., eating health diets), which could contribute to improved physical health (Carrico, Johnson, Colfax, \& Moskowitz, 2010; Harrison et al., 2000) Similarly, positive emotions are associated with social support, which may facilitate improved physical health outcomes (Algoe \& Stanton, 2012). Additional mechanisms hypothesize that positive emotions affect physical health by decreasing physiological responding to stress and improving recovery from stress (Fredrickson \& Levenson, 1998). For example, research has shown that humor promotes decreased physiological reactivity to stress (Newman \& Stone, 1996; Harrison et al., 2000). In one study, participants viewed three types of films, exciting, humorous, and didactic. Researchers found that watching a humorous film resulted in decreased cardiac output and a lengthening of the pre-ejection period, which suggests a decreased stress response (Harrison et al., 2000). In contrast, the exciting film resulted in increased sympathetic nervous system activity as demonstrated by increases in systolic blood pressure, heart rate, cardiac output, and a shortening of the pre-ejection period. Other research found that a giving a humorous narration of a stressful film resulted in an increase in skin temperature and a decrease in skin conductance, while heart rate showed a non-significant decreasing trend (Newman \& 
Stone, 1996). Further evidence supporting the hypothesis that positive emotions contribute to decreased physiological responding to stress can be found in the cardiovascular reactivity literature. The cardiovascular reactivity (CVR) hypothesis proposes that elevated cardiovascular responses to stressors, stimuli that induce stress, increase the risk of developing CVD. Therefore, if positive emotions consistently reduce physiological responding to stress, then it is likely that physical health would be improved.

\section{Cardiovascular Reactivity and Recovery}

The cardiovascular reactivity (CVR) hypothesis, originally proposed by Obrist (1976), has been conceptualized both as a causal factor in the development of CVD and as the mechanism through which stress affects cardiovascular health (Schwartz et al., 2003). CVR may represent a potential mechanism explaining the relation between positive emotions and health. According to this perspective, positive emotions may promote a reduction in the magnitude of CVR (i.e., lower heart rate and blood pressure response) to stress. If the experience of positive emotions consistently resulted in this reduced physiological reactivity, then over time, the experience of positive emotions may promote improved physical health. Currently, there is evidence that CVR may be at least one mechanism through which stress, "the nonspecific response of the body to any demand made upon it" (Selye, 1973, p. 692), impairs cardiovascular health (Chida \& Steptoe, 2010). Despite a large amount of research examining the reactivity hypothesis, a number of challenges have emerged in this body of literature that remain to be addressed. Two of the challenges in this literature are the limited generalizability of lab findings to naturalistic settings and the duration of the external stimuli that elicit stress responses typically used in these research studies (Schwartz et al., 2003). Regarding the former issue, there is limited evidence of generalizability of CVR to naturalistic settings, which limits the support 
for the hypothesis that CVR is a causal mechanism through which psychological factors lead to CVD (Schwartz et al., 2003). Certainly, in order to support a causal association between the magnitude of cardiovascular responses to stress and CVD, one would need to show that the magnitude of cardiovascular responses measured in laboratory or clinic settings reflected the magnitude of cardiovascular responses to real life stressors. Regarding the second issue, in contrast to real life stressors, the duration of laboratory stressors are typically short (e.g., 3-5 min), and consequently, it is difficult to conclude that exaggerated responses to brief challenges in the laboratory explain the pathogenesis of CVD that occurs over years, if not decades, of life (Schwartz et al., 2003). A more recent conceptualization of the reactivity hypothesis has extended it by proposing that prolonged activation of the stress response may be more important than the magnitude of the stress response in explaining how exposure to stress in life may lead to CVD (Pieper \& Brosschot, 2005).

Prolonged activation of cardiovascular arousal consists of: recovery, reoccurrence, and anticipation (Pieper \& Brosschot, 2005). According to these authors, recovery refers to the measurement of duration of cardiovascular activation after a stressor is terminated. Reoccurrence of activation refers to a reactivation of the stress response following a recovery period, often occurring if an individual engages in rumination (i.e., dwelling on the stressor). The final type of prolonged activation, anticipation, involves activation of the stress response as an individual remains vigilant preparing for subsequent stressors.

Consistent with the original findings supporting the hypothesis that the magnitude of cardiovascular reactivity to stress was associated with disease outcomes, multiple studies have now demonstrated that prolonged activation (i.e., delayed recovery from stress) is positively related to risk for CVD (Borghi, Costa, Boschi, Mussi, \& Ambrosioni, 1986; Cole, Blackstone, 
Pashkow, Snader, \& Lauer, 1999; Everson, G.A., Goldberg, \& Salonen, 1996; Stewart \& France, 2001; Treiber et al., 2001). Studies examining recovery from stress found that delayed systolic blood pressure (SBP) recovery from stress was a greater risk factor for the development of hypertension five years later than the magnitude of SBP reactivity to stress (Borghi et al., 1986). Further, impaired SBP recovery following several stressor tasks (i.e., cold pressor, tourniquet ischemia, and step exercise) has been shown to be associated with elevated resting SBP at follow-up (Stewart \& France, 2001). Similar findings have been demonstrated for delayed heart rate $(\mathrm{HR})$ recovery. For example, individuals with slowed HR recovery following physical and emotional stressors exhibited elevated resting HR four years later (Treiber et al., 2001). In addition, delayed HR recovery following an exercise task predicted all-cause mortality six years later (Cole et al., 1999).

These effects are not limited to recovery from stress alone as studies examining the effects of anticipation of subsequent stressors demonstrated similar findings. For example, one study demonstrated that elevated blood pressure in anticipation of an exercise task predicted future hypertension status (Everson et al., 1996). Another study found that increases in left ventricular hypertrophy (thickening of the ventricle wall) were related to elevated anticipatory BP (Kamarck et al., 2000). Cumulatively, these results indicate that prolonged activation of arousal through either delayed recovery or anticipation to laboratory stressors represent potentially pathogenic components of the physiological response to stress (Pieper \& Brosschot, 2005). Further, these studies suggest that prolonged activation may be a stronger predictor of future CVD than the magnitude of CVR.

Although most CVR studies include a recovery period, recovery data are often not analyzed; therefore, no systematic conceptualization or definition of recovery exists and multiple 
strategies are used for purposes of data analysis (Linden, Earle, Gerin, \& Christenfeld, 1997). The most common methods for conceptualizing and analyzing recovery are to examine (a) the time to recovery, or (b) recovery at a fixed time point (Christenfeld, Glynn, \& Gerin, 2000). Time to recovery examines the amount of time that elapses between the end of a stressor and the return to resting physiological levels. Recovery at a fixed time point examines the magnitude of reactivity remaining after a specified period of time (e.g., five minutes after stress has been removed). A third, but less common way of conceptualizing recovery, is total carryover, which examines the amount of residual arousal that occurs during the recovery period. Total carryover is similar to examining the area between the recovery curve and resting level. In one study, time to recovery, recovery at a fixed time point, and total carryover were found to be unreliable measures of individual differences in recovery with test-retest correlation coefficients ranging from .11 to .24 for time to recovery and total carryover respectively (Christenfeld et al., 2000). Another method of conceptualizing and analyzing recovery data is to examine area under the curve (AUC). AUC is a superior method for analyzing time to recovery as it controls for the rate of decline in physiological responding across the entire recovery period (Linden et al., 1997). A smaller area under the curve indicates greater physical recovery (i.e., reduced physiological responding). Therefore, using area under the curve techniques may be the most reliable method for conceptualizing and analyzing recovery data.

\section{The Undoing Effect of Positive Emotions}

Considering the evidence demonstrating a relation between delayed recovery from stress and CVD, exploring methods to facilitate recovery from stress is an area of interest. Although it is well known that exposure to situations that evoke NA result in increased HR and BP, less is known regarding how emotional factors moderate recovery from stress. In contrast, very little is 
known about how exposure to situations that evoke PA influence the magnitude and patterning of $\mathrm{HR}$ and $\mathrm{BP}$ reactivity to stress and recovery from stress. It is quite possible that PA does not exert an influence on the magnitude of the cardiovascular reaction to stress, but rather permits the rapid recovery from stressful encounters. The hypothesis that explains how PA exerts a positive effect on health is called the undoing hypothesis (Fredrickson \& Levenson, 1998). Specifically, the undoing hypothesis suggests that positive emotions experienced following a stressor can improve physiological recovery by reducing the duration of cardiovascular reactivity (Fredrickson \& Levenson, 1998). Multiple studies have demonstrated support for the undoing hypothesis (Dowd, Zautra, \& Hogan, 2010; Fredrickson \& Levenson, 1998; Fredrickson, Mancuso, Branigan, \& Tugade, 2000; Kraft \& Pressman, 2012; Ong \& Allaire, 2005; Papousek et al., 2010; Sokhadze, 2007; Steptoe, Gibson, Hamer, \& Wardle, 2007; Tugade, Fredrickson, \& Barrett, 2004; Yuan, McCarthy, Holley, \& Levenson, 2010).

In the initial study of this type, Fredrickson and Levenson (1998) demonstrated that contentment- and amusement-film conditions promoted faster physiological recovery following exposure to fear evoking stimuli when compared to neutral- and sad-film conditions. Fredrickson et al. (2000) replicated and extended earlier findings in two separate samples. In Sample 1, contentment- and amusement-film conditions produced faster physiological recovery following a social stressor task (i.e., preparing a speech) as compared to neutral- and sad-film conditions. In addition, a second sample demonstrated a similar pattern of findings with the contentment- and amusement-film conditions promoting faster cardiovascular recovery as compared to the neutralfilm condition. Another study, which experimentally-induced positive emotions, examined positive, stressful, and neutral interactions among married couples (Yuan et al., 2010). The authors found that during physiological down-regulation events (i.e., periods of low 
physiological arousal or recovery), the ratio of positive to total emotions was greater as compared to non-down-regulation events. This finding suggests that positive emotions contributed to improved physiological recovery from stress. Although a number of studies that have experimentally-induced PA demonstrated support for the undoing hypothesis, it is important to note that a majority of these studies employed external stimuli to evoke PA (e.g., amusement films). Utilizing external stimuli to evoke PA strengthens the internal validity of these studies because the stimuli are standardized and the experimenter can be sure that the participant engages in them. However, external validity, specifically the generalizability of findings to one's daily life is limited. That is, not many people watch amusing film segments following coping with a life stressor. To address issues of generalizability, researchers have begun to examine naturally-occurring PA by either measuring the range of PA following stress presentations and comparing those with higher or lower levels of PA or inducing PA through recollections of personally-relevant events.

Studies examining naturally-occurring PA include two main types, laboratory-based studies and ambulatory studies. In two studies, Tugade et al. (2004) found that the experience of naturally-occurring positive emotions fully mediated the relation between trait resilience and cardiovascular recovery following a speech task. In another lab-based study, experiencing high state PA predicted faster SBP recovery following a stressor task than among those who experienced low state PA (Dowd et al., 2010). Ambulatory studies demonstrated similar findings. For example, one study found that ecological momentary assessments (EMA) of naturally-occurring PA were negatively associated with SBP and DBP levels during recovery from daily life stress (Steptoe et al., 2007). Further, this study found that PA, as measured by the Positive and Negative Affect Schedule (PANAS), was associated with a more rapid SBP 
recovery, but not DBP or HR recovery from stress. It should be noted that the physiological measures and EMA ratings of PA were obtained at two separate time points in this study, and therefore, one cannot assume a causal relation. Studies measuring naturally-occurring PA enhance the generalizability of findings to real-life settings. However, these studies exhibit less internal validity than laboratory-based studies, and therefore, it is difficult to confirm the directional relation between variables from their findings.

Several studies employed the experimental induction of memories of PA (e.g., recalling a happy or positive memory) to bridge the gap between experimentally-induced PA through external stimuli and studies measuring naturally-occurring PA (Hannesdottir, 2007; Purdum, 2010). Hannesdottir's (2007) study compared physiological recovery among three groups: positive-film condition, happy-memory recall condition, and a neutral-film condition. There were no significant differences between the groups, suggesting that physiological recovery was not improved by PA induced through either a film condition or from a memory recall of a happy moment condition. Similarly, Purdum (2010) examined how recall of a positive event affected physiological recovery. Participants were randomized to one of two groups: recalling a positive event before recalling a negative event or recalling a positive event after recalling a negative event. Purdum (2010) was interested in examining the stress buffering and recovery effects of positive emotions. No differences in physiological reactivity or recovery were found. A potential problem with these two studies is their reliance on presentation of internal stimuli (e.g., recalling a pleasant event) that cannot be easily verified by the experimenter. Studies that have used external stimuli (e.g., viewing amusing films or mimicking specific facial expressions) have been more consistent in supporting the undoing effect of PA. 


\section{The Facial Feedback Hypothesis}

One type of experimental method that can be used to induce positive emotions reliably stems from studies examining the facial feedback hypothesis. The facial feedback hypothesis proposes that emotional states can be induced through activation of specific facial muscles (Tourangeau \& Ellsworth, 1979). Three hypotheses postulating how facial expressions may contribute to or modulate emotional experience (Davis, Senghas, \& Ochsner, 2009; Tourangeau \& Ellsworth, 1979) have been devised and tested: the necessity, sufficiency, and modulation hypotheses. The necessity hypothesis, which has largely been discredited, proposes that emotional experience is not possible without emotional expression or feedback from facial muscles (Keillor, Barrett, Crucian, Kortenkamp, \& Heilman, 2002). According to this perspective, the experience of anger requires portrayal of the facial features of anger (e.g., furrowed brow, glaring eyes). The sufficiency hypothesis proposes that activation of facial muscles alone is capable of producing the associated emotional experience (Ekman, Levenson, \& Friesen, 1983). Unlike the necessity hypothesis, the sufficiency hypothesis has some support in the literature (Levenson \& Ekman, 2002). According to the final hypothesis, the modulation hypothesis, emotional experience can be modulated through facial expression (Strack, Martin, \& Stepper, 1988). Critically, modulation of emotional experience is possible even if the emotional experience results from an external stimulus and not through facial muscle activation.

Multiple studies have examined the effect of activating specific facial muscles on emotional experience. Often, the Duchenne or genuine smile is investigated. The Duchenne smile involves activation of the zygomaticus major and orbicularis oculi muscles and has been demonstrated to occur more frequently during viewing of pleasant films compared to viewing unpleasant films (Davidson, 1992). In addition, producing a Duchenne smile during pleasant 
scenes or humorous cartoons is known to result in more experience of PA than groups producing a non-Duchenne smile (Soussignan, 2002). Overall, these studies suggest that facial activation of various muscles modulates the experience of emotions.

Further, evidence supporting the facial feedback muscles is found in literature examining how botulinum toxin (BOTOX) affects emotional experience. Botulinum toxin is a type of neurotoxin that can paralyze muscles (Dolly \& Aoki, 2006). One study (Davis, Senghas, \& Ochsner, 2010) compared the effects of Botox injections on emotional experience to the effects of Restylane, an injectable hyaluronic acid filler that does not affect facial muscles (Brandt \& Cazzaniga, 2007). The study involved two sessions one occurring within 8 days before injections and the other occurring 14-24 days after injections. Participants in both groups were exposed to films evoking mildly positive, positive, negative emotions, and non-emotional film clips. Participants rated their emotions using the PANAS and the Beck Depression Inventory (BDI). Results found that participants in the Botox group demonstrated a less intense emotional experience to the mildly positive film clips relative to the Restylane group. However, no other differences were found. A major limitation of this study was the use of a quasi-experimental design in which participants were not randomized to treatments and chose which injection they received. The authors proposed that the activation of facial muscles may not be the sole determinant of emotional experience, but other research examining the facial feedback hypothesis offers stronger evidence for its effects on emotional experience.

Findings based on the facial feedback hypothesis have led toward the exploration of how activation of specific facial muscles influences physiological recovery from stress or emotional provocation. Two studies have explored the effect of facial muscle manipulation on physiological recovery and found evidence supporting the modulating effect of facial activation 
of specific muscles (Fredrickson \& Levenson, 1998; Kraft \& Pressman, 2012). In one study, participants who engaged in spontaneous smiling demonstrated faster physiological recovery from a sad-film induction $(M=35.9 \mathrm{~s})$ as compared to nonsmilers $(M=56.4 \mathrm{~s} ; p=.028)$ (Fredrickson \& Levenson, 1998). Although participants were not assigned to smile and no smile conditions in this study, the effects of spontaneous smiling are perhaps important to consider as this demonstrates greater generalizability to naturally-occurring response tendencies. In a study with stronger methodological control, recovery following two stressor tasks (i.e., star-tracer and cold pressor tasks) was examined. Participants were assigned to one of three conditions (a Duchenne-smile group, a standard-smile group, and a neutral-group). The neutral-group held chopsticks in their mouths to maintain a relaxed expression and the two smile groups activated specific facial muscles. Awareness of smiling was also manipulated in the two smile groups by instructing half of the participants to smile during the instruction periods. Aware standard smilers demonstrated the lowest HR during recovery for the star-tracer $(M=65.75 \mathrm{bpm})$ and cold pressor tasks $(M=66.33 \mathrm{bpm})$ as compared to all other groups. Nonaware standard smilers demonstrated the highest HR during recovery for the star-tracer $(M=72.73 \mathrm{bpm})$ and cold pressor tasks $(M=71.43 \mathrm{bpm})$ as compared to all other groups. Following the star-tracer task, nonaware Duchenne smilers $(M=66.50 \mathrm{bpm})$ and aware Duchenne smilers $(M=67.40 \mathrm{bpm})$ demonstrated lower HR recovery as compared to the neutral group $(M=71.36 \mathrm{bpm})$ and the nonaware standard smilers. BP findings were not reported but the authors noted that the differences between groups were largely not significant. The pattern of recovery was relatively similar across groups following the two stressor tasks. This study suggests that activation of facial muscles to stimulate smiling improves physiological HR recovery from stress. 


\section{Purpose of the Current Study and Specific Aims}

Current evidence suggests that delayed or impaired physiological recovery from stress is a stronger predictor of cardiovascular health than the magnitude of cardiovascular reactivity (Borghi et al., 1986; Cole et al., 1999; Kamarck et al., 2000; Stewart \& France, 2001; Treiber et al., 2001). Therefore, examining physiological recovery and how physiological recovery can be improved is critical in understanding the relation between stress and CVD. Inducing PA may represent one method for facilitating physiological recovery from stressors. Several studies have explored the undoing effect of positive emotions with mixed findings (Fredrickson \& Levenson, 1998; Fredrickson et al., 2000; Hannesdottir, 2007; Kaczmarek, 2009). In general, studies utilizing external stimuli, such as amusing films or positive images, lack personal relevance to participants. The lack of personal relevance and nature of these external stimuli (individuals may not be able to view amusing films following stressful events in daily life) limits the generalizability of these effects. In contrast, studies utilizing internal stimuli typically induce PA through recollection of personal memories. However, these tasks are particularly problematic because it is not possible to ascertain if participants have engaged in the internal task of recalling a memory. Further, studies utilizing internal stimuli to induce PA have not demonstrated much support for the undoing effect (Hannesdottir, 2007; Purdum, 2010). Although there are several problems with previous methods used to induce PA, there is evidence, albeit mixed, supporting the undoing effect on physiological recovery from stress.

This study aimed to examine the role of two different strategies of inducing PA to determine which method facilitated $\mathrm{HR}$ and $\mathrm{BP}$ recovery from stress the most in documenting the undoing effect. Consistent with previous work (Kraft \& Pressman, 2012), one strategy induced PA by manipulating the facial portrayal of smiling based upon the facial feedback 
hypothesis of emotion (i.e., a Duchenne-smile manipulation). The other strategy involved the recollection of a personally-relevant pleasant event. This approach bridged a gap in the literature by modifying a task that is typically considered internal, recalling a pleasant event, and pairing it with an external cue associated with the pleasant event (i.e., personally-relevant photographs). As noted above, the use of recollections of personally-relevant pleasant events is complicated by the inability of the experimenter to ensure that the participants were engaging in the task. Through the use of personally-relevant photographs provided by participants, the procedure assured that participants were exposed to the stimulus and examined whether it influenced physiological recovery similar to previous studies that supported the undoing effect (Fredrickson \& Levenson, 1998, Study 1; Yuan et al., 2010). By examining the optimal method for facilitating physiological recovery and demonstrating the "undoing" of stress or negative affect by PA, then eventually, it will be possible to develop interventions that may ultimately contribute to a reduced risk of developing CVD among high risk patients.

The Duchenne-smile manipulation was selected as one method for inducing PA in the current study based on research that has shown that smiling is associated with more rapid physiological recovery from stress (Kraft \& Pressman, 2012). For example, in one study, engaging in Duchenne smiling facilitated HR recovery. In the current study, participants in this condition were trained to activate the zygomaticus major and orbicularis oculi muscles to create the Duchenne smile during exposure to a stressful task.

The second method for inducing PA in the current study was a modification of a strategy typically thought to involve exposure to internal stimuli (e.g., recalling a pleasant memory). Specifically, this modification included presentation of personally-relevant photographs provided by participants during task completion. Through this modification, exposure to PA-eliciting 
stimuli was assured. The personally-relevant photograph condition is a novel recovery task that has not been previously examined in the literature. In brief, participants in this condition provided photographs of a positive event (e.g., celebrating a birthday). In this regard, the photographs served as an external reminder of a positive event. Previous studies employed recall of positive event conditions (Hannesdottir, 2007; Purdum, 2010) to induce PA; however, because the extent to which participants adhered to instructions to recall the pleasant event could not be measured reliably, it was not possible to guarantee that participants recalled the pleasant event and thus exposed themselves to the positive emotions associated with recalling the event. Additionally, it was challenging for study participants to balance time spent recalling a pleasant event with cognitive effort required to complete the stressful task. The photograph condition in the current study addressed these problems by instructing participants to attend to the photographs when the photographs were presented following each mental arithmetic problem. Further, participants engaged in the stressor tasks and the positive emotion induction tasks simultaneously as one of the aims of the current study was to conduct a partial replication of Kraft and Pressman (2012). In the Kraft and Pressman paper, participants maintained the positive emotion induction task during the stressor tasks. The current study involved use of two positive emotion induction tasks and therefore, it was determined that engagement in the stressor tasks and the positive emotion induction tasks would occur concurrently.

Mental arithmetic is a commonly used and well-validated stressor task. In general, research has demonstrated that mental arithmetic produces increases in sympathetic nervous system activity coupled with parasympathetic nervous system withdrawal (Allen, 2000). Typically, increases in sympathetic activity and moderate increases in HR and BP are observed during task completion, which indicates a beta-adrenergic response (Allen, 2000; Allen, Boquet, 
\& Shelley, 1991). There are two general types of mental arithmetic, countdown tasks vs. computer-generated tasks (Allen, 2000). The countdown task is perhaps most well-known. In this task, participants subtract a smaller number from a large number (Allen, 2000). For example, participants may be instructed to engage in a Serial $7 \mathrm{~s}$ task in which they subtract seven from a large number (e.g., 5678). In the computer-generated task, participants are presented with math problems on a computer, instructed to solve the problems, and indicate the correct responses (Allen, 2000). For the purposes of the current study, a computer-generated arithmetic task developed and tested by Salvia, Guillot, and Collet (2013) was utilized to ensure that the mental arithmetic problems alternated with the PA-inducing stimuli. Salvia et al.'s task includes two sets of math problems, easy and difficult, each involving two mathematical operations (e.g., addition, subtraction, multiplication, or division). In their study, Salvia et al. found that completion of math problems produced increases in sympathetic nervous system activity, and that difficult math problems resulted in longer HR responses than easy math problems. A similar, but modified version of this task was used in the current study.

A majority of the previous studies examining the undoing effect of PA with respect to physiological reactivity to stress or recovery from stress examined a single strategy for inducing PA (i.e., recalling a pleasant event, watching amusing movies, engaging in a Duchenne smile). Only one study induced PA using two different strategies, recalling a pleasant memory and watching amusing movies (Hannesdottir, 2007). However, in this study, no differences in reactivity or recovery were observed for either strategy in comparison to a control group. Given that other research has demonstrated beneficial effects of viewing amusing films and the problems noted with using recollection strategies, it is difficult to determine whether the lack of effects observed in this study reflected the unreliability of the finding or the nature of the 
strategies selected to induce PA. To examine whether the type of strategy used to induce PA moderates the magnitude of the effect, it becomes necessary to compare strategies within a single study. In contrast to most prior work, this study involved two distinct strategies for inducing PA. Further, by comparing two different strategies that can be done during completion of stressful tasks like mental arithmetic, it is possible to examine whether the magnitude of the undoing effect increases as more PA is experienced. For example, it is unknown whether participants who view personally-relevant photographs while recalling a pleasant event while smiling experience a more rapid physiological recovery than participants engaging in either strategy alone.

To examine these research questions, the current study employed a 2 (Duchenne-smile, no smile) x 2 (personally-relevant photographs, non-personally relevant photographs) betweensubjects design. Using this type of design enabled the direct comparison of two methods of inducing PA to determine if one method was superior at inducing PA. Further, the comparison between the two methods enabled an examination of whether one method was superior at promoting improved physiological recovery. It is possible that both engaging in the Duchennesmile and viewing the personally-relevant photographs promoted physiological recovery. If participants in the Duchenne-smile group (Smile group) experienced a more rapid physiological recovery than those in the no smile group (No-smile group), this would be referred to as the Smile main effect. If participants in the personally-relevant photographs group (Happy group) experienced a more rapid physiological recovery than those in the non-personally relevant photographs group (Neutral group), this would be referred to as the Event main effect. It is also possible that there will be an interaction between smiling and viewing the personally-relevant photographs (i.e., interaction effect). As there are a limited number of studies examining physiological recovery using the manipulation of facial muscles to induce PA and no studies 
examining the effect of personally-relevant photographs to induce PA, data are insufficient to make specific hypotheses. However, based upon the theoretical foundation of the undoing effect, as established by Fredrickson and Levenson (1998), the induction of PA was expected to result in a more rapid recovery of $\mathrm{HR}$ and $\mathrm{BP}$ reactions to the mental arithmetic stress.

\section{Method}

\section{Participants and Sample Size}

This study included two phases, an online screening phase and a laboratory phase. The purpose of the online screening phase was to identify participants that were eligible for the laboratory phase of the study. Prior to conducting the main study, a pilot study was conducted to examine the procedure and stimuli used in the laboratory phase of the study (see Appendix A). Participants in the pilot study, online screening phase, and laboratory phase of the study included male and female students from West Virginia University. In addition, only students who were 18 years or older were included as study participants.

A power analysis using $\mathrm{G}^{*}$ Power 3.1 .3 was conducted to determine the sample size required for the laboratory phase of the study. The sample size for the laboratory phase was determined by entering the study design as an a priori, ANCOVA: Fixed effect, special, main effects, and interactions" with an effect size $f$ of $0.38, \alpha=.05$, and power $=80 \%$ (Faul, Erdfelder, Buchner, \& Lang, 2009). The effect size used in the power analysis is based on previous studies examining physiological recovery from stress. Several studies found medium to large effect sizes (Fredrickson \& Levenson, 1998, Study 1 and Study 2). For example, in one study examining the effect of amusement- and contentment-inducing films on physiological recovery, a large effect size (omega-squared $=.27$ ) was observed (Fredrickson \& Levenson, 1998, Study 1). Similarly, in a study examining the effect of spontaneous smiling, a medium effect size (omega-squared = 
.05) was found (Fredrickson \& Levenson, 1998, Study 2). The power analysis indicated that a sample size of 57 participants was sufficient to detect medium to large effect sizes. However, as the laboratory phase of the study included four groups, the sample size was increased to 60 to ensure that there would be an equal number of participants in each group (15 participants in each group). Participants were excluded from the laboratory phase of the study based on the following criteria: nicotine use, major chronic health conditions, use of medications affecting heart rate or blood pressure, any serious mental health problem or use of psychoactive medications that could interfere with dependent variables.

SONA Screening Phase. During the screening phase, 805 undergraduate students (206 men and 599 women, $M_{\text {age }}=19.5$ years, $S D=3.09$ years) completed brief questionnaires on the SONA data collection site (see Table 1). However, one participant reported his age as 17 years old and therefore, was excluded from analyses. This resulted in a sample of 804 undergraduate students (205 men and 599 women, $M_{\text {age }}=19.5$ years, $S D=3.09$ years). The sample included 694 white participants, 36 black participants, 15 Asian participants, 51 participants that described their race as other/biracial, and 8 participants that did not disclose their race. Additionally, 739 participants described their ethnicity as not Hispanic or Latino and 32 participants described their ethnicity as Hispanic or Latino. Participants received one unit of extra credit for participating in the online phase. The average length of time it took for participants to complete the online questionnaires was 20.02 minutes $(S D=9.57)$. From this sample, 397 participants were determined to be eligible for the laboratory phase of the study based on the aforementioned inclusion criteria and were invited to participate in the laboratory phase. The 17-year student was inadvertently included in this group and invited to participate; this deviation from protocol 
was reported to the IRB and his data have been deleted. Additional demographics from the SONA screening sample are shown in Table 2.

Laboratory Phase. The sample invited to complete the laboratory phase of the study included 61 participants ( 14 men and 47 women with a $M_{\text {age }}=19.4$ years, $S D=1.46$ ) recruited from the SONA screening phase sample (see Table 1). The Smile-Happy group included 14 participants, the Smile-Neutral and No-smile-Happy Groups included 15 participants and the Neutral Group included 17 participants. The sample included 50 white participants, 5 black participants, 3 Asian participants, 3 participants that described their race as other/biracial. Additionally, 1 female participant described her ethnicity as Hispanic or Latino. See Table 2 for additional demographic information. Participants received one unit of extra credit and $\$ 10$ for participating in the laboratory phase of the study.

\section{Experimental Design}

The study employed a $2 \times 2$ between-subjects design. The first between subjects factor, Smile, was based on the facial feedback hypothesis (Smile, No-smile). The second between subjects factor, Event, was exposure to photographs of a pleasant or neutral event (Happy, Neutral). Dependent variables included cardiovascular parameters as well as measures of positive and negative affect.

\section{Measures}

Demographic Form. A short demographic form, previously used in the Behavioral Physiology Lab (e.g., Cavanagh, 2013; Prentice, 2009), was used in the study. The form measures participants' general health behaviors and includes questions about age, sex, height, weight, race/ethnicity, year in school, and parental socioeconomic status (see Appendix B). 
Positive and Negative Affect Schedule. The Positive and Negative Affect ScheduleExpanded Form (PANAS-X) includes 60-items measuring positive and negative affect on a 5point Likert-type scale $(1=$ very slightly or not at all to $5=$ extremely $)$. The PANAS-X measures general PA and NA as well as measuring 11 specific affects, including PA (joviality, selfassurance, and attentiveness) and NA (fear, hostility, guilt, and sadness). Additionally, the PANAS-X allows for measurement of other affective states, including shyness, fatigue, serenity, and surprise (Watson \& Clark, 1994). For the purposes of this study, the general PA and NA scales were examined. As discussed previously, PA is defined as a state of pleasurable engagement, which is characterized by enthusiasm and alertness. NA, in contrast, is defined as a state of unpleasurable engagement and is characterized by subjective distress (Crawford \& Henry, 2004; Watson, Clark, \& Tellegen, 1988). The PANAS-X has been validated with different temporal instructions, including moment instructions ("at the present moment"), the past year ("during the past year") and in general ("on average") instructions. In this study, the on average instructions were used in the online SONA phase of the study to examine trait PA and NA. In addition, the moment instructions were used in the laboratory phase of the study to examine state PA and NA. Alpha reliabilities among undergraduate samples for PA when using the on average (.87) and moment instructions (.88) have demonstrated strong internal consistency reliability. Similarly, for NA, when using the on average (.85) and the moment instructions, alpha reliabilities (.85) have demonstrated strong internal reliability (Watson \& Clark, 1994). Regarding validity, Watson and Clark (1994) examined correlations between the fear subscale of the PANAS-X and the tension-anxiety scale from the Profile of Mood States (POMS) (.85), the hostility subscale of the PANAS-X and the anger-hostility of the POMS (.91), sadness subscale of the PANAS-X and the depression-dejection scale of the POMS (.85), the 
fatigue subscale of the PANAS-X and the fatigue scale of the POMS (.89), and the general PA scale of the PANAS-X with the vigor scale of the POMS (.86). Discriminant validity was also examined by comparing the sadness, fear, and hostility subscales of the PANAS-X to the Beck Depression Inventory (BDI), the depression scale of the Hopkins Symptom Checklist (HSCL), Center for Epidemiological Studies Depression Scale (CES-D), State-Trait Anxiety Inventory (STAI), and the State-Trait Anger Scale (STAS). Discriminant correlations ranged from .26 to .83 (David Watson \& Clark, 1992). Most correlations ranged from .30 to .60. In the current study, Cronbach's alphas for General NA during the resting, stress, and recovery periods were $.54, .84$, and .62 , respectively. In the current study, Cronbach's alphas for General PA at resting, stress, and recovery periods were $.85, .78$, and .89 .

Mental Arithmetic Questionnaire. In a study using a computer-generated arithmetic stressor task, participants completed a mental arithmetic questionnaire (Salvia, Guillot, \& Collet, 2013). This 14-item questionnaire assessed participants' ease, spontaneity, and frequency of using mental arithmetic. Participants responded to items on a 6-point Likert-type scale $(1=d o n$ ' $t$ agree at all to $6=$ completely agree . The purpose of the questionnaire, developed by Salvia et al. (2013), was to categorize participants as those with an "affinity" for mental arithmetic and those who engaged in "avoidance" of mental arithmetic. Participants in the affinity group had higher mean scores (4 to 6) and participants in the avoidance group had lower mean scores (1 to 3). In the current study, this questionnaire was not used to categorize participants. However, it was used to determine if it was necessary to control for participants' affinity to or avoidance of mental arithmetic (see Appendix C). For the purposes of this study, the anchors of the Likerttype scale $(1=$ strongly disagree to $6=$ strongly agree $)$ were adjusted. In addition, the language used in several items was also changed (e.g., using elementary school instead of primary school 
or using blackboard instead of chalk + slate). This questionnaire was originally developed for use in France and therefore, the language in several items needed to be modified. Instructions for completing the questionnaire were also developed for the study, as they were not provided in the original study.

Post-Experiment Questionnaire. Participants in all four groups completed a PostExperiment Questionnaire following the recovery period. Participants in the Smile-Happy and No-smile-Happy Groups completed the Post-Experiment Questionnaire-A (see Appendix J). Participants in Smile-Neutral and the No-smile-Neutral Groups completed the Post-Experiment Questionnaire-B (see Appendix K). Both the A and B versions of the Post-Experiment Questionnaire included questions assessing the difficulty, comfort, and distractibility of the stressor and recovery tasks. Additionally, participants completed a manipulation check question regarding the purpose of the chopsticks. The two versions of the questionnaire differed in regard to the questions assessing responses to the images (personally-relevant photographs vs. neutral photographs) that were presented. Participants in Groups 1 and 3 answered questions about the event and memories associated with the photographs they provided. In contrast, participants in Groups 2 and 4 answered questions about the neutral images they were presented.

\section{Behavioral Observation}

Behavioral observations were conducted for participants in the pilot and main laboratory phases of the study. The purpose of the behavioral observations was to examine if participants maintained the Duchenne-smile and non-smile poses throughout the stressor period. Coders used one still image of each the Duchenne-smile and non-smile poses as examples to identify the correct positioning for each manipulation. There was no established criterion for coders during training. To examine if participants maintained the correct facial manipulations, two coders 
coded the length of time that participants maintained the Duchenne-smile or the non-smile pose. Coders then coded study sessions as a yes if participants maintained the correct pose for 4 of the 5 minute stressor task and as a no if participants were not able to maintain the pose for 4 of the 5 minute stressor task. To assess coder agreement on the behavioral measure, study sessions were double-coded and Kappa coefficients between raters were calculated.

\section{Apparatus}

Heart Rate. Heart rate and heart rate variability (HRV) were measured using a Polar Model RS800 Heart Rate Monitor (Lake Success, New York). A sensor device was attached around the participants' chest that measured HR and transmitted data to a USB receiver connected to a computer. The ECG responses were monitored throughout the study. Previous research has demonstrated that the Polar Monitor is a valid measure of HR (Goodie, Larkin, \& Schauss, 2000). Polar Monitor software was used to clean HR recordings and artefacts due to poor recordings were removed.

Heart Rate Variability. Spectral analysis for two measures of HRV, high frequency heart rate variability (HF HRV) and low frequency heart rate variability (LF HRV), were conducted using Kubios (version 2.1) HRV software. HF HRV reflects parasympathetic nervous system activity, whereas LF HRV reflects both sympathetic and parasympathetic nervous system activity (Tarvainen \& Niskanen, 2008). The Kubios HRV Analysis Software program, which is a valid method for analyzing HR and HRV, was used to analyze HRV data (Niskanen, Tarvainen, Ranta-Aho, \& Karjalainen, 2004; Tarvainen \& Niskanen, 2008). A spectrum setting of $4 \mathrm{~Hz}$ interpolation rate is the standard setting for examining normal HRV (Tarvainen \& Niskanen, 2008). 
Blood Pressure. Blood pressure was measured using an Industrial and Biomedical Sensors, Inc. SD-700A automated sphygmomanometer (Waltham, MA). A microphone is contained inside the occluding cuff to detect Korotkoff sounds and was positioned over the brachial artery. The device electronically controls cuff inflation and deflation and detects Korotkoff sounds.

\section{Experimental Stimuli}

Stressor Task. The procedure and stimuli for presenting mental arithmetic developed by Salvia et al. (2013) were adapted and utilized in the proposed study (see Appendix D for math problems and instructions provided to participants prior to beginning of the stressor task). For the purposes of this study, the calculations used in this study were the same as the calculations used by Salvia et al. (2013); however, the instructions provided to participants were modified. In Salvia's procedure, participants were exposed to 24 easy and 24 difficult math problems, presented in a random order. Each math problem was presented in a specific sequence, including a Preparation phase (i.e., black cross presented on screen for $5 \mathrm{~s}$ ), the Calculation phase (e.g., $((44+24) / 2)$ for $20 \mathrm{~s}$ for easy problems and $30 \mathrm{~s}$ for difficult problems, the Results phase (i.e., response accuracy and reaction time presented as a global score for $10 \mathrm{~s}$ ), and the Rest phase (i.e., blank screen presented for $15 \mathrm{~s}$ for easy problems and $30 \mathrm{~s}$ for difficult problems). In the Calculation phase, participants entered their response. After participants entered their response the Results phase began. In the Results phase, reaction time and response accuracy were combined to form a global score as a means of maintaining participants' motivation. In the present study the procedure used by Salvia was modified as described below.

In the current study, the Visual Basic computer program was used to create the math stressor program. Before beginning the math problems, participants were given instructions, 
indicated their understanding of the instructions for the stressor task, and began holding the Duchenne-smile or no-smile pose. The mental arithmetic stressor task followed a specific sequence, including an Anticipation phase (i.e., personally-relevant or non-personally relevant photographs were presented on screen for $10 \mathrm{~s}$ ) and the Calculation phase (e.g., a mix of easy and difficult math problems were presented for $20 \mathrm{~s}$ and $30 \mathrm{~s}$ respectively). During the Calculation phase, a countdown timer appeared in the corner of the computer screen informing participants of the time available to complete the math problem. If participants failed to provide an answer during the allotted time, the math problem closed and the anticipation phase began again. Participants received feedback regarding if their answers were correct or incorrect each time they entered an answer. If participants failed to enter an answer for three consecutive math problems, they were prompted with the following statement "Please attempt to do your best on the math problems." Additionally, all participants received the following feedback: "Please remember your training" halfway through the stressor task. The total time to complete the stressor task was 5 minutes. At the end of the stressor task, participants were able to view the total number of math problems they answered correctly and the total number of math problems they attempted to answer.

Duchenne Smile Manipulation. Two types of facial muscle manipulations were used in this study, the Duchenne smile and the nonsmile group. As discussed previously, studies testing the facial feedback hypothesis demonstrated that the Duchenne-smile consistently produced pleasant emotions (Soussignan, 2002). In the present study, participants randomized to the Duchenne-smile group positioned chopsticks in their mouths in a sideways position so that the zygomaticus major and orbicularis oculi muscles were activated. Participants randomized to the Duchenne-smile group were presented with stimuli for positioning assistance and training 
instructions. In the nonsmile group, participants were instructed to hold the chopsticks gently while maintaining a relaxed face. Participants randomized to the nonsmile group were presented with stimuli for positioning assistance and training instructions. Previous research employed this stimulus to ensure adherence to the Duchenne-smile and adherence to the nonsmile or neutral position (Kraft \& Pressman, 2012).

Photographs. In the present study, two types of images were used, three neutral images from the International Affective Picture System and three personally-relevant photographs. The IAPS contains over 700 photographs of positive, negative, and neutral stimuli and was developed by Lang and colleagues (Lang, Bradley, \& Cuthbert, 1995). Photographs contained in the IAPS have been well validated and norms have been established (Lang et al., 1995). A recent study reexamined the IAPS norms and found that the norms were relatively similar to norms established previously (Libkuman, Otani, Kern, Viger, \& Novak, 2007). As these norms are similar to earlier research, a neutral photograph from the IAPS is a validated and reliable stimuli (see Appendix F). The neutral images (chair, mug, and baskets) selected from the IAPS were rated as relatively similar in terms of valence and arousal. Valence and arousal were measured using the Self-Assessment Manikin (SAM), which uses three graphical figures to indicate emotional reactions (Lang, Bradley, \& Cuthbert, 2008). Responses can include one of the three graphical figures or responses can be rated between two graphical figures. Therefore, each dimension can be rated on a 9-point scale with higher ratings representing higher levels of pleasure and arousal and lower ratings representing lower levels of pleasure and arousal. For valence, the chair received a mean rating of $4.96(S D=1.12)$, the mug received a mean rating of $4.98(S D=0.96)$, and the baskets received a mean rating of $4.99(S D=1.18)$ (Lang et al., 2008). For arousal, the chair received a mean rating of $2.83(S D=2.00)$, the mug received a mean rating 
of $2.66(S D=1.82)$, and the baskets received a mean rating of $2.60(S D=1.78)$. These ratings indicate that the chair, mugs, and baskets are rated as neutral stimuli in terms of valence and relatively low in arousal.

Participants randomized to the personally-relevant photograph group provided researchers with three photographs of one pleasant memory prior to the laboratory session. In this group, participants received an email message instructing them to select three personallyrelevant photographs of a pleasant event (e.g., birthday, graduation, etc.) and forward them to the experimenter prior to the laboratory session (see Appendix $\mathrm{G}$ for instructions to participants randomized to this group).

\section{Procedure}

Screening Phase. Participants read a cover letter describing the risk and benefits of participating in the study when they logged onto the SONA site. Participants who agreed to continue with the study then completed several questionnaires: Demographics Form, Mental Arithmetic Questionnaire, and PANAS-X. Eligible participants were contacted through email to schedule individual laboratory sessions. Prior to being contacted to schedule a laboratory session, participants were randomized to groups using a website that generates random numbers and lists (Random.org). Participants randomized to the personally-relevant photograph groups received a request to provide the three personally-relevant photographs of a pleasant memory. All participants were instructed to refrain from exercise and consumption of alcohol or caffeine for a minimum of 2 hours prior to the laboratory session.

Laboratory Phase. At the beginning of the laboratory session, participants completed IRB approved consent forms and physiological equipment (Polar Monitor and AccuTracker) was attached. Next, participants listened to a description of the purpose of the study (see Appendix 
H). Based on the randomization, participants received training and instructions regarding the recovery tasks in which they would engage. For example, if randomized to the Duchenne-smile groups, participants received training and positioning assistance to ensure that the zygomaticus major and orbicularis oculi muscles were activated (see Appendix E for Duchenne-smile and neutral-smile group positioning assistance and training instructions; see Appendix G for photograph instructions). In addition, participants also received instructions regarding the stressor task (see Appendix H). A similar study description has been used in previous studies (Kraft \& Pressman, 2012; Strack et al., 1988).

After the physiological equipment was attached, participants entered an Adaptation Period of five minutes. Participants then entered a Resting Period of ten minutes. Participants then completed the PANAS-X at the end of the Resting Period. Participants then began the 5 minute stressor task. The mental arithmetic task and the personally-relevant or neutral photographs were presented on a laptop using the Visual Basic program. Participants engaged in their respective facial muscle manipulation during the stressor task and removed the chopsticks after completing the mental arithmetic task. At the end of the stressor task, participants removed the chopsticks and completed the PANAS-X for a second time. Next, participants entered a 10

minute Recovery period. After completion of the Recovery period, participants completed a final PANAS-X, physiological equipment was detached, and participants were debriefed and compensated for their time (see Appendix I).

\section{Results}

\section{Data Reduction and Assumption Checks}

The data were analyzed using Excel Microsoft Office 2010 and PASW Statistics 19 and 21. First, basic data cleaning steps were conducted. Data cleaning steps included examining 
parameter distributions for normality and examining correlations between demographic variables (e.g., age, sex, body mass index, and math affinity) and cardiovascular parameters. Means and standard deviations of participant characteristics and dependent variables are shown in Tables 13 and the correlation matrix between demographic and cardiovascular variables are shown in Table 4.

\section{Missing Data and Removal of Invalid Blood Pressure Recordings.}

Blood pressure measures were examined according to the criteria established by Marler, Jacob, Lehoczky, and Shapiro (1988) and measures that met these criteria were removed. This criterion suggests removing unacceptably high or low blood pressures and low pulse pressures (pulse pressures less than $30 \mathrm{~mm} \mathrm{Hg}$ ). Pulse pressure is calculated by subtracting diastolic blood pressure from systolic blood pressure. If cases of pulse pressures were less than $30 \mathrm{~mm} \mathrm{Hg}$, the associated systolic and diastolic blood pressures were removed from future analyses. Only one pulse pressure was found to be less than $30 \mathrm{~mm} \mathrm{Hg}$ and therefore, the associated systolic and diastolic blood pressure values were removed from subsequent analyses. Additionally, there were a total of $8.28 \%$ data points across all measures of SBP and DBP for the resting, stressor, and recovery periods that were considered missing data due to equipment malfunction.

Resting Physiological Parameters. Prior to calculating average resting cardiovascular parameters, for both HR and BP, individual data points for each segment of the initial rest period were analyzed using one-way repeated measures analyses of variance (ANOVAs) to assess stability of resting physiological state during the entire resting period (see Table 5). For purposes of this analysis, missing data points were imputed by averaging the other BP values individually for participants rather than using mean imputation. Although proximal BP values may be a better estimate of each missing data point than the group means, this method resulted in a greater 
number of missing cases as opposed to the mean imputation method. For example, after imputing missing data based on proximal BP values there remained 72 and 70 missing instances of SBP and DBP respectively. Therefore, the mean imputation method was used. For resting SBP and DBP, a total of $6.45 \%$ of data points were imputed. After imputations, one case remained missing for resting SBP and DBP because no data were available for the entire resting period. For resting SBP, a Greenhouse-Geisser correction was applied because of unequal variances. The ANOVA was not significant, $F(3.64,218.31)=1.52, p=.20$, indicating that SBP was stable across the initial resting period. This was confirmed by the conducting the same analysis using the proximal imputation method for SBP, $F(5,46)=1.61, p=.18$.

For resting DBP, the ANOVA was also not significant, $F(4.27,255.97)=2.08, p=.08$. However, the same analysis using proximal value imputation methods revealed a significant main effect, $F(5,46)=2.72, p=.03)$. An examination of pairwise comparisons revealed a significant difference between Minute $4(M=63.7 \mathrm{~mm} \mathrm{Hg}, S D=9.77)$ and Minute $10(M=67.0$ $\mathrm{mm} \mathrm{Hg}, S D=8.59)(p=.026)$. As the significant difference occurred between minutes 4 and minutes 10 and because minute 10 was a higher DBP reading, this suggested that DBP at Minute 10 did not reflect resting DBP, potentially due to an anticipation effect of the upcoming task or from fatigue or frustration with the lengthy resting period. Consequently, Minute 10 values of DBP were not used in calculating resting DBP and resting DBP was determined by averaging DBP values from Minutes $0,2,4,6$, and 8 .

As discussed previously, the method of imputing missing HR cases using proximal HR values was considered. However, after imputation using the proximal HR values, there remained 112 instances of missing HRs and therefore, it was determined that mean imputation would be a preferred method to retain a greater number of cases. For resting HR, 2.10\% of data points were 
imputed using the same method employed for BP imputation. Resting HR data for three participants were entirely missing across the resting period and therefore, could not be imputed. For resting HR, the ANOVA was not significant, $F(2.96,168.61)=2.49, p=.06$. However, using the proximal imputation method, a significant effect was observed, $F(9,46)=4.14, p=$ .001. Pairwise comparisons revealed several significant differences: Minute $1(M=78.2, S D=$ 12.61) was significantly lower than Minute $2(M=80.8, S D=13.36)(p=.002)$, Minute $4(M=$ $80.7, S D=13.45)(p=.017)$, and Minute $10(M=81.4, S D=14.80)(p=.016)$, and Minute 2 was significantly higher than Minute $3(M=78.9, S D=13.06)(p=.047)$. Because Minute 1 was less than Minutes 2, 4, and 10 this did not indicate a problem with adaptation to the resting phase of the study that would have resulted in the initial HR being higher than the following HRs. Additionally, because the difference between Minutes 2 and 3 did not reflect a gradual change in HR across the baseline period, all resting HR values were retained for calculating resting HR. Resting HR was calculated by averaging HRs across Minutes 1 - 10.

Based upon these analyses, individual values for each respective physiological measure were averaged to derive single measures of resting HR, SBP, and DBP. For HRV measures, inter-beat intervals during the entire 10-min resting period were subjected to spectral analysis using the Kubios software to derive single measures of resting HF-HRV and LF-HRV. Because three participants were missing HR data for the entire resting period, values of HRV for these three participants could not be calculated.

Physiological Measures during Stress. Like resting measures, individual data points for each segment of the 5-minute task period were analyzed using one-way repeated measures ANOVAs to assess stability of reactivity during the math task (see Table 6). However, as participants were engaged in a stressful task (mental arithmetic task), it was expected that there 
would be differences between physiological measures. For SBP and DBP values during the task, $12.90 \%$ of data points were imputed, but three data points could not be imputed because they occurred within the same participant. For SBP during the task, the ANOVA was significant, $F(1.73,102.31)=4.62, p=.02$ [using proximal imputation, $F(2,45)=4.18, p=.02$ ] . Specifically, pairwise comparisons indicated that Minute $0(M=120.8 \mathrm{~mm} \mathrm{Hg}, S D=12.65)$ was significantly greater than Minute $4(M=118.5 \mathrm{~mm} \mathrm{Hg}, S D=11.41)(p=.02)$.

For DBP during the task, the ANOVA was also significant, $F(2,118)=4.35, p=.02$, [using proximal imputation, $F(2,45)=6.22, p=.004$ ]. Specifically, pairwise comparisons indicated that Minute $0(M=69.6 \mathrm{~mm} \mathrm{Hg}, S D=10.36)$ was significantly less than Minute $4(M$ $=73.1 \mathrm{~mm} \mathrm{Hg}, S D=8.36)(p=.008)$.

For HR during the task, the ANOVA was not significant, $F(2.82,158.06)=2.38, p=.08$ [using proximal imputation, $F(4,52)=3.30, p=.02$ ]. However, there was one significant pairwise comparison. Minute $1(M=79.3, S D=12.91)$ was significantly lower than Minute $2(M$ $=81.0, S D=12.60)(p=.03)$.

These findings revealed relatively small changes from the beginning to the end of the stressor task, and because these changes throughout the task were small, all values were retained for calculating mean reactivity values during the task. The averages for SBP and DBP during the task period included readings from Minutes 0, 2, and 4 and the average for HR during the stressor period included readings from Minutes 1 - 5. Like the rest period, inter-beat intervals during the entire task period were subjected to spectral analysis to derive single measures of HFHRV and LF-HRV during the task.

Physiological Measures during Recovery from Stress. For analyzing recovery data, areas under the curve (AUC) were calculated for each cardiovascular parameter for each 
participant. AUC was determined by calculating excursions based on the formulas used in previous studies (Friedberg, Suchday, \& Shelov, 2007; Neumann, Waldstein, Sellers, Thayer, \& Sorkin, 2004). For the current study, the formula for AUC for blood pressure was: Excursion = $(0.5 * 120) *((\mathrm{SBP} / \mathrm{DBP}$ at recovery $\min 0)+(2 * \mathrm{SBP} / \mathrm{DBP}$ at recovery $\min 2)+(2 * \mathrm{SBP} / \mathrm{DBP}$ at recovery $\min 4)+(2 * \mathrm{SBP} / \mathrm{DBP}$ at recovery $\min 6)+(2 * \mathrm{SBP} / \mathrm{DBP}$ at recovery $\min 8)+$ (SBP/DBP at recovery min 10) - SBP/DBP at baseline * 600)). For SBP and DBP recovery, $7.80 \%$ of data points were imputed and there were no missing cases for SBP and DBP recovery.

The final SBP analyses of recovery included 13 cases in the Smile-Happy group, 15 cases in the Smile-Neutral group, 15 cases in the No-smile-Happy group, and 17 cases in the Neutral group. The final DBP analyses of recovery included 12 cases in the Smile-Happy group, 15 cases in the Smile-Neutral group, 15 cases in the No-smile-Happy group, and 17 cases in the Neutral group.

Heart Rate. The AUC formula for HR was: Excursion $=(0.5 * 60) *((\mathrm{HR}$ at recovery $\min 1)+(2 * \mathrm{HR}$ at recovery $\min 2)+(2 * \mathrm{HR}$ at recovery $\min 3)+(2 * \mathrm{HR}$ at recovery $\min 4)+$ $(2 * \mathrm{HR}$ at recovery $\min 5)+(2 * \mathrm{HR}$ at recovery $\min 6)+(2 * \mathrm{HR}$ at recovery $\min 7)+(2 * \mathrm{HR}$ at recovery $\min 8)+(2 * \mathrm{HR}$ at recovery $\min 9)+(\mathrm{HR}$ at recovery $\min 10)-\mathrm{HR}$ at baseline $*$ 600)). For HR recovery, only $1.29 \%$ of cases were imputed but HRs for 4 cases were entirely missing. The final HR analyses included 12 cases in the Smile-Happy group, 12 cases in the Smile-Neutral group, 15 cases in the No-Smile-Happy group, and 17 cases in the Neutral group.

Heart Rate Variability. By convention, HRV is generally log transformed. For the current study, natural log transformations were conducted on all HRV data for the resting and task periods. Resting measures of both LF HRV and HF HRV were available for 61 cases and task measures were available for 59 cases. The AUC HRV formula was: Excursion $=\left(0.5^{*} 300\right)$ 
$*((\mathrm{HRV}$ at stressor $)+(2 * \mathrm{HRV}$ at recovery $\min 5)+(\mathrm{HRV}$ at recovery $\min 10)-\mathrm{HRV}$ at baseline * 600). The AUC formula for HRV was calculated with and without natural log transformations of HRV stressor and HRV recovery. The final LF HRV analyses included 12 cases in the Smile-Happy group, 12 cases in the Smile-Neutral group, 13 cases in the No-SmileHappy group, and 16 cases in the Neutral group. The final HF HRV analyses included 12 cases in the Smile-Happy group, 13 cases in the Smile-Neutral group, 14 cases in the No-Smile-Happy group, and 17 cases in the Neutral group.

Testing statistical assumptions. ANCOVAs are relatively robust to violations of data assumptions (Field, 2009). However, the data assumptions for ANCOVA, assumptions of normality, homogeneity of variance, random independent samples, linearity (linear relationships between dependent variable and covariates), homogeneity of regression slopes, and that the covariate is independent of the treatment effects (Tabachnick \& Fidell, 2013) were examined prior to conducting the main analyses.

Normality was examined for all demographic variables and covariates. There was no evidence of non-normality among most demographic variables, with the exception of age and BMI. Both of these variables were skewed, so they were log transformed to normalize these distributions.

Affect, including measures of PA and NA, was measured three times in the current study, after the completion of the initial resting period, the stressor period, and the recovery period. General NA was positively skewed for the resting, stressor, and recovery periods (standardized skewness value was greater than the recommended cutoff of 3.29). General PA was normallydistributed. Initially square-root transformations were applied to all NA variables. Although this transformation corrected the skewness for General NA for the resting and recovery periods, 
General NA for the stressor period remained positively skewed. Therefore, a log (base 10) transformation was applied to all NA variables which corrected the skewness for General NA stressor period. Means and standard deviations for PA and NA variables are reported in Tables 8. As noted above, transformations were done on measures of HRV and the NA scale of the PANAS-X to reduce any problems associated with violating this assumption (see Table 3). It should be noted that although these transformations did not entirely normalize the distributions of several variables, including heart rate variability, outliers were not removed due to the loss of study power associated with such a strategy. Means and standard deviations of transformed and untransformed covariates and cardiovascular parameters for the resting, stressor, and recovery periods are shown in Table 3.

The Shapiro-Wilk test of normality and the standardized skewness value were used to determine if AUC data was normally distributed within each group. For SBP AUC, the ShapiroWilk test was significant $(p=.016)$ for the Neutral group. The Shapiro-Wilk test was also significant $(p \leq .001)$ for DBP AUC for the Smile-Happy group. For HR AUC, the Shapiro-Wilk test was significant for the Neutral group $(p=.005)$. Additionally, the Shapiro-Wilk test was significant for LF HRV AUC for the Smile-Neutral group $(p \leq .001)$ and for the Neutral group $(p$ $=.009)$. However, the Shapiro-Wilk test was not significant for HRV AUC data when using the natural-log transformed data to calculate AUC.

To further examine normality within each group a measure of standardized skewness was calculated (skewness statistic/standard error of skewness). According to Tabachnick and Fidell (2013) standardized skewness of the absolute value of $\geq 3.29$ are considered skewed. The combined evidence from the Shapiro-Wilk test and the standardized skewness value was used to determine if the data was normally distributed across the four groups for each dependent 
measure. The standardized skewness value was below the recommended 3.29 value for all groups for SBP and HR AUC, indicating that the data was normally-distributed; therefore, no transformations were conducted on the SBP or HR AUC data. The standardized skewness variable was 4.94 for DBP AUC for the Smile-Happy group, which is greater than the suggested cutoff for the standardized skewness value. However, the Shapiro-Wilk's test for DBP AUC was non-significant and an analysis of DBP AUC for outliers demonstrated no evidence of outliers. Therefore, no transformations were conducted on DBP AUC. The standardized skewness variable was -5.07 for LF HRV AUC (using the untransformed data) for the Smile-Neutral group, which is greater than the suggested cutoff for the standardized skewness value. The standardized skewness values for LF and HF HRV AUC (using the transformed data) were below the recommended cutoff value for all groups. Therefore, the main analyses were conducted with both the untransformed and the transformed HRV AUC data.

The assumption of homogeneity of variance was examined using Levene's test. The assumption of homogeneity of variance is violated in cases in which Levene's test is significant. There were two cases in which Levene's test was significant, HR AUC and LF HRV AUC (natural-log transformed). However, some statisticians suggest that other methods are better for examining homogeneity of variance (Field, 2009). One alternative method is to examine the variance ratio (the ratio between the largest and smallest variances) across groups for each dependent variable (Field, 2009). If the variance ratio is found to be less than two then the assumption of homogeneity of variance has not been violated. There were a few cases in which the variance ratios were slightly above the recommended cutoff of 2, including SBP AUC (2.67), DBP AUC (2.12), HF HRV AUC (natural-log transformed) (9.14), and LF HRV AUC (natural- 
$\log$ transformed) (3.01). The variance ratio for HR AUC (37.58) was much larger than the suggested variance ratio of $<2$.

The cumulative evidence suggested that the assumption of homogeneity of variance was not violated for SBP AUC, DBP AUC, or HF HRV AUC (untransformed and transformed). The evidence from the variance ratio and Levene's test indicates that the assumption of homogeneity of variance was violated for HR AUC and LF HRV AUC (untransformed and transformed).

The assumption of linearity between the dependent variables and covariates was examined through correlations. Correlations were conducted between the dependent variables and measures considered as potential covariates. There was no evidence of non-linear relations. Although several correlations were not significant between resting and respective AUC measures, this was not expected because the resting level is used in the calculation of AUC. However, because resting values need to be included as covariates to control for the law of initial values, they were included in the main analyses despite the lack of significant correlations.

The final two assumptions, homogeneity of regression slopes and the assumption that the covariate is independent of the treatment effects, were then examined. Homogeneity of regression slopes was examined through an ANCOVA for each dependent variable with group as the independent variable and with respective covariates included. Interaction effects between the independent variables and the covariates (e.g., Group X SBP baseline) were examined. If the interaction effect was significant then this indicated that the assumption of homogeneity of regression slopes had been violated. There were several cases of significant interaction effects, indicating that the assumption of homogeneity of regression slopes was violated for these three analyses. For HR AUC, there was a significant interaction effect between the smile variable and HR stressor $(p=.04)$. For LF HRV AUC (transformed), there was a significant interaction effect 
between the event variable and LF HRV baseline $(p=.05)$. Also, for HF HRV AUC (transformed), there was a significant interaction effect between the event variable and HF HRV baseline $(p=.03)$. Therefore, main analyses were conducted both with and without covariates in these cases. There were no other significant interaction effects for the other dependent measures, which indicated that the assumption of homogeneity of regression slopes was not violated for these other dependent measures. Finally, a one-way ANOVA with group as the independent variable and the covariates as the outcomes was conducted to determine if the covariates were independent of the treatment effect. The ANOVA for each covariate was not significant indicating that this assumption was not violated.

Overall, the data for this study met the majority of the statistical assumptions required to conduct an ANCOVA. The assumption of independence of the covariate and the treatment effect were supported for all of the data. Further, the assumptions of normality, homogeneity of variance, linearity, and homogeneity of regression slopes were supported by a majority of the data and there were only a few cases in which these assumptions were violated. Finally, as previously discussed the ANCOVA is relatively robust to violations of statistical assumptions. Therefore, the main analyses, consisting of between-subjects ANCOVAs, were conducted.

Correlations among Dependent Variables and Covariates. Univariate correlations were conducted to examine the relations between the dependent variables, demographic variables (age, sex, and BMI), math affinity (as measured by the Mental Arithmetic Questionnaire), affect measures, and cardiovascular parameters during rest, task, and recovery periods (see Table 4).

Significant correlations were found between all measures of SBP and Sex $(r s=-.30,-.26$, and -.30 for resting, task, and recovery periods). Further examination of SBPs among men and women confirm that men had higher SBPs $(M=117.3 \mathrm{~mm} \mathrm{Hg}, S D=10.37)$ than women $(M=$ 
$111.1 \mathrm{~mm} \mathrm{Hg}, S D=7.68)$, and the significant correlation between resting DBP and BMI ( $r=$ .35) confirms that greater body mass was associated with higher resting DBP. Although sex differences in resting SBP were observed, sex was not considered a covariate in subsequent analyses, because resting SBP was already being entered as a covariate. Regarding the relation between BMI and resting DBP, BMI was entered as a covariate in subsequent analyses of DBP. Math affinity was significantly correlated with resting LF HRV $(r=.26)$, but inversely correlated with LF HRV AUC $(r=-.41)$. Regarding correlations between cardiovascular parameters and self-reported measures of PA and NA yielded one consistent finding, SBP was associated with PA during each phase of the experiment $(r \mathrm{~s}=.32, .29$, and .28 for resting, task, and recovery periods).

Behavioral Coding. Two trained behavioral coders reviewed the five minute task period during which participants maintained a Duchenne-smile or a neutral expression. Each coder indicated whether participants maintained the correct expression for four of the five minutes. Kappa coefficients were used to determine the level of agreement between the two coders. Kappa coefficients between 0.21-0.40 suggest fair agreement and Kappa coefficients between 0.41-0.60 suggest moderate agreement. Analysis of Kappa coefficients in the pilot study included 7 cases. In the pilot study, Kappa $=.09, p=.81$, which suggests poor agreement. In the laboratory phase of the study, Kappa $=.44, p=.038$, which suggests moderate agreement. The Kappa coefficient for the pilot study may have been lower than the Kappa coefficient from the laboratory phase of this study because the sample size was substantially smaller in the pilot study. Additionally, during the pilot phase of the present study an internal camera within the laptop was used for recording behavioral observations. The camera used during the pilot study resulted in poor quality videos and occasionally resulted in a complete loss of video signal from 
the camera. As a result of the lost video signal, only 7 participants from the pilot study had sufficient video length and quality for recording behavioral observations. Further, behavioral observations could have been affected by the overall poor quality of video obtained during the pilot study. To improve the video quality and prevent loss of video recordings, an external camera was used for the main laboratory phase.

The Kappa coefficients for the Duchenne-smile groups and the No-smile groups for the main laboratory study were also examined separately to determine if there were any differences in coders' ability to accurately code for a smile as compared to a neutral expression. The Kappa coefficients were examined for the two groups and included 60 cases that were double-coded. One case was not double coded and therefore was excluded from analyses examining the Kappa coefficients. For the Duchenne-smile groups (28 cases), Kappa $=.374, p=.004$. In contrast, for the No-smile groups (32 cases), Kappa $=.518, p=.003$. Thus, raters were much more consistent in evaluating portrayal of the neutral expression versus the Duchenne-smile. Overall, although inter-rater reliability was far from excellent, the results from the behavioral observations indicated that data from all participants were considered appropriate to include in the analyses. It should be acknowledged, however, that determining whether the correct facial portrayal of the Duchenne smile or neutral expression occurred for 4 of the 5 minutes of the task period did not reflect a simple discrimination for raters.

Performance on Math Problems. The mean number of math problems that participants were presented with was $7.7(S D=2.26)$ and the mean number of math problems that participants answered correctly was $.21(S D=.20)$, reflecting that $21.5 \%$ of the problems were answered correctly $(S D=20.41 \%)$. To determine whether comparable task performance was exhibited among participants randomized to the four groups, a 2 X 2 [Smile (Duchenne smile, 
No smile) by Event (Happy, Neutral)] ANOVA was conducted on the percentage of math problems answered correctly. The ANOVA revealed no significant main effects for Smile, $F(1$, $50)=.32, p=.58$, or Event, $F(1,50)=.16, p=.70$, and no significant interaction effect, $F(1,50)$ $=.09, p=.93$ (see Table 7). Additionally, a Smile by Event ANOVA was conducted to examine group differences in scores on the Mental Arithmetic Questionnaire. The ANOVA revealed no significant main effects for Smile, $F(1,54)=.19, p=.67$, or Event, $F(1,54)=.27, p=.60$, and no significant interaction effect, $F(1,54)=2.17, p=.15$. Finally, a Smile by Event ANCOVA was conducted to examine group differences in performance on the stressor task with scores on the Mental Arithmetic Questionnaire included as a covariate. The ANOVA revealed no significant main effects for Smile, $F(1,47)=.31, p=.58$, or Event, $F(1,47)=.07, p=.80$, and no significant interaction effect, $F(1,47)=.08, p=.78$

Reactivity to Math Problems. To demonstrate that the stressor task was effective in producing increases in physiological responses, a paired-samples t-test was conducted for each dependent variable comparing the measure obtained during the task period with its respective pre-task resting value (see Table 8). The stressor task was expected to produce sympathetic activation and therefore, a comparison of the physiological measures during the stressor period to the baseline period should demonstrate this sympathetic activation. The results of these analyses indicated that there was evidence of SBP and DBP reactivity during the stressor period. SBP was significantly higher during the stressor period $(M=119.5 \mathrm{~mm} \mathrm{Hg}, S D=11.17)$ as compared to the pre-task resting period $(M=112.8 \mathrm{~mm} \mathrm{Hg}, S D=8.49, t(59)=-7.83, p \leq .001)$. Similarly, DBP was significantly higher during the stressor period $(M=71.6 \mathrm{~mm} \mathrm{Hg}, S D=8.07)$ than during the baseline period $(M=65.1 \mathrm{~mm} \mathrm{Hg}, S D=7.66, t(59)=-6.83, p \leq .001$. HR during the stressor period was slightly higher $(M=80.3 \mathrm{bpm}, S D=12.42)$ than HR during the resting 
period $(M=79.5 \mathrm{bpm}, S D=12.75)$; however, this change was not significant, $t(56)=-.94, p=$ .35. Likewise, there were no significant change in LF HRV, $t(57)=.02, p=.99$, or HF HRV from the resting period to the task period, $t(57)=.81, p=.42$.

Paired-samples t-tests were also conducted to examine the change in NA and PA from resting to stressor periods. As expected NA was significantly higher during the stressor period $(M=1.15, S D=.13)$ than NA during the resting period $(M=1.07, S D=.07, t(60)=-6.07, p \leq$ .001. Interestingly, PA during the resting period was significantly higher $(M=23.33, S D=6.47)$ than PA during the stressor period $(M=21.35, S D=5.68, t(59)=2.72, p=.009$.

Because the primary purpose of this study was to determine whether two specific manipulations for inducing positive affect enhanced recovery from stress, parameters for which no significant stress response was observed were not examined further. Based upon the pattern of these analyses of change, several dependent variables of interest failed to demonstrate significant change from the pre-task resting period, including HR, LF HRV, and HF HRV. Because no HR or HRV response to the math stress was observed in this study, analyses of recovery from stress were not conducted on HR or HRV.

\section{Primary Study Analyses on Cardiovascular Parameters}

Initial Resting Period. Prior to conducting the primary study analyses, it was important to test whether participants assigned to the four experimental groups differed during the initial resting period or during the stress period on any cardiovascular parameter. Resting cardiovascular levels were each analyzed using a 2 x 2 [Smile (Duchenne-smile, Non-smile) X Event (Happy, Neutral)] between-subjects ANOVA (see Table 9). For SBP during the resting period there were no significant main effects for Smile, $F(1,57)=.36, p=.55$, or Event, $F(1,57)$ $=.006, p=.94$, or significant Smile by Event interaction, $F(1,57)=.001, p=.98$. 
For DBP during the resting period, there were no significant main effects for Smile, $F(1$, $57)=.16, p=.77$, or Event, $F(1,57)=.09, p=.77$. However, there was a significant interaction effect of Smile and Event for DBP, $F(1,57)=5.37, p=.02$. Simple main effects demonstrated a significant difference between the Smile-Neutral $(M=68.0 \mathrm{~mm} \mathrm{Hg}, S D=7.65)$ and Neutral groups $(M=62.8 \mathrm{~mm} \mathrm{Hg}, S D=8.70), F(1,57)=3.87 p=.05$ (see Table 10). There were no differences between the Smile-Happy $(M=63.0 \mathrm{~mm} \mathrm{Hg}, S D=4.96)$ and No-smile Happy groups $(M=66.6 \mathrm{~mm} \mathrm{Hg}, S D=7.63), F(1,57)=1.76 p=.19$.

Reactivity to the Task. For purposes of analyzing reactivity to the math task, comparable Smile by Event ANCOVAs were conducted on BP values during the task using pre-task resting periods as covariates (see Table 11). For SBP, there was no significant main effect for Smile, $F(1,55)=.13, p=.72$ or significant interaction effect between Smile and Event, $F(1,55)=.77$, $p=.39$. However, there was a significant main effect of Event, with the personally-relevant photograph groups (Smile-Happy, $M=124.3 \mathrm{~mm} \mathrm{Hg}, S D=12.01$; No-Smile-Happy, $M=119.3$ $\mathrm{mm} \mathrm{Hg}, S D=12.45$ ) demonstrating higher SBP reactivity than the neutral photograph groups (Smile-Neutral, $M=118.0 \mathrm{~mm} \mathrm{Hg}, S D=9.33$; No-Smile-Neutral, $M=117.5 \mathrm{~mm} \mathrm{Hg}, S D=$ $10.72), F(1,55)=4.30, p=.04$.

For DBP reactivity to the task, there was a significant main effect for Smile, $F(1,55)=$ $7.56, p=.008$ with the Smile groups (Smile-Happy, $M=72.6 \mathrm{~mm} \mathrm{Hg}, S D=7.28$; Smile-Neutral, $M=75.8 \mathrm{~mm} \mathrm{Hg}, S D=7.41$ ) demonstrating greater DBP reactivity as compared to the No-Smile groups (No-Smile-Happy, $M=69.6 \mathrm{~mm} \mathrm{Hg}, S D=9.02$; Neutral, $M=68.7 \mathrm{~mm} \mathrm{Hg}, S D=7.13$ ). There were no significant main effects for Event, $F(1,55)=.28, p=.60$, or significant interaction effects, $F(1,55)=.09, p=.77$, for measures of DBP task reactivity (See Table 11). 
Recovery from the Task. The primary study analysis involved a 2 x 2 [Smile (Duchennesmile, Non-smile) X Event (Happy, Neutral)] between-subjects analysis of covariance (ANCOVAs) on the area under the curve during recovery for each cardiovascular parameter (see Table 12). Resting cardiovascular values and cardiovascular reactivity levels were entered into the analyses as covariates. A significance level of .01 was used to examine results.

For SBP recovery, there was no significant main effect for Smile, $F(1,54)=.34 p=.57$, or Event, $F(1,54)=3.80 p=.06$, or for the interaction effect between Smile and Event, $F(1,54)$ $=.27 p=.61$. For DBP recovery, there was no significant main effect for Smile, $F(1,52)=.06$, $p=.82$ or Event, $F(1,52)=1.15, p=.29$, or the interaction effect, $F(1,52)=.49, p=.49$. As discussed previously, there was no evidence of HR or HRV reactivity; therefore, HR and HRV recovery were not examined.

Examination of Recovery as a Return to Baseline. In an attempt to compare results from the present study with previous research examining the undoing effect, recovery was also examined as the length of time to return to initial resting levels of SBP and DBP. Cases in which participants returned to resting level by Minute 0 of the recovery period were coded as 1 , by Minute 2 were coded as 3, by Minute 4 were coded as 5, by Minute 6 were coded as 7, by Minute 8 were coded as 9 , and by Minute 10 were coded as 11 . Participants who failed to return to resting levels during the entire recovery period were also coded as 11 . To examine recovery as time to return to resting levels, $2 \times 2$ between-subjects ANOVAs were conducted (see Table 13).

For time to return to resting level SBP, there was no significant main effect for Smile, $F(1,57)=1.99, p=.16$, or Event, $F(1,57)=.91, p=.34$, or for the interaction effect between Smile and Event, $F(1,57)=.02, p=.88$. For time to return to resting DBP, there was no 
significant main effect for Smile, $F(1,57)=.60, p=.44$, or Event, $F(1,57)=.00, p=.95$, or the interaction effect, $F(1,57)=1.86, p=.18$.

Measures of Affect. To determine if the stimuli and tasks influenced participant's levels of affect, 2 (Smile) X 2 (Event) ANOVAs were conducted for both PA and NA for each period (see Table 14). For NA during the resting period, there were no significant main effects for Smile, $F(1,57)=.77, p=.38$, or Event, $F(1,57)=.09, p=.77$, or interaction effect, $F(1,57)=$ $.06, p=.81$. For NA during the stressor period, there were no significant main effects for Smile, $F(1,57)=.34, p=.56$, or Event, $F(1,57)=.02, p=.88$, or the interaction effect, $F(1,57)=.11$, $p=.75$. For NA during the recovery period, there were no significant main effects for Smile, $F(1,57)=.01, p=.95$, or Event, $F(1,57)=.16, p=.69$, or the interaction effect, $F(1,57)=.11$, $p=.75$.

For PA during the resting period, there were no significant main effects for Smile, $F(1$, $57)=.41, p=.52$, or Event, $F(1,57)=.02, p=.90$, or the interaction effect, $F(1,57)=.66, p=$ 42. For PA during the stressor period, there were no significant main effects for Smile, $F(1,56)$ $=1.89, p=.17$, or Event, $F(1,56)=.13, p=.72$, or the interaction effect, $F(1,56)=.31, p=.58$. For PA during the recovery period, there were no significant main effects of Smile, $F(1,57)=$ $1.75, p=.19$, or Event, $F(1,57)=.17, p=.68$, or the interaction effect, $F(1,57)=.04, p=.85$.

Post Experiment Questionnaire Ratings. To examine the effects of the math task, the facial muscle manipulation task, and the photographs, several 2 (Smile) X 2 Event ANOVAs were conducted for items on the Post Experiment Questionnaire that were completed by all participants (see Table 15). First, participants in all groups rated the difficulty of the smile manipulation task, how tired the task made participants, and how uncomfortable the task was 
(see Table 15). Participants in Groups 1 and 3 rated the Duchenne-smile task and participants in Groups 2 and 4 rated the no-smile task.

Difficulty of manipulation. Participants rated the difficulty of maintaining the manipulation of facial muscles with placements of the chopsticks on a Likert-type scale with anchors of $0 \%=$ Not at all difficult to $100 \%=$ Very difficult . The ANOVA demonstrated no significant main effect of Smile, $F(1,56)=1.41, p=.24$, or Event, $F(1,56)=.46, p=.50$, or interaction effect, $F(1,56)=.23, p=.63$. These results indicate that all participants rated the task similarly as a moderately difficult task.

Tiredness of manipulation. Participants rated how tired the placement of the chopsticks made them on a Likert-type scale with anchors of $0 \%=$ Not at all tired to $100 \%=$ Very tried. The ANOVA demonstrated no significant main effect of Smile, $F(1,56)=1.53, p=.22$, or Event, $F(1,56)=.002, p=.97$, or interaction effect, $F(1,56)=.12, p=.73$. These results suggest that all groups rated the task as eliciting a moderate level of tiredness.

Uncomfortableness of manipulation. Participants rated how uncomfortable the placement of the chopsticks made them on a Likert-type scale with anchors of $0 \%=$ Not at all uncomfortable to $100 \%=$ Very uncomfortable. The ANOVA demonstrated no significant main effect of Smile, $F(1,57)=.04, p=.85$, or Event, $F(1,57)=.54, p=.47$, or interaction effect, $F(1,57)=.91, p=.35$. This indicates that all groups rated the task as resulting in a moderate level of discomfort.

Distraction. Participants also rated how distracted they were by the personally-relevant or non-personally relevant photographs when completing the math problems on a Likert-type scale from $0 \%=$ Not at all distracted to $100 \%=$ Very distracted (see Table 15$)$. The ANOVA demonstrated no significant main effect for Smile, $F(1,56)=.25, p=.62$, or interaction effect, 
$F(1,56)=.11 p=.74$. However, there was a significant main effect for Event, $F(1,56)=4.40, p$ $=.04$. Participants in the Smile-Happy $(M=39.64, S D=19.26)$ and No-smile Happy groups $(M$ $=38.67, S D=22.64)$ rated the personally-relevant photographs groups as more distracting than the Smile-Neutral $(M=29.29, S D=26.81)$ and the Neutral groups' ratings of the neutral photographs $(M=24.41, S D=21.50)$.

Participants also rated eight items assessing types of thoughts that occurred when completing the mental arithmetic stressor task on a Likert-type scale from $1=$ Strongly Disagree to 7 = Strongly Agree. A 2 (Smile) X 2 Event ANOVA was conducted on each item (see Table 16).

Pleasant Thoughts. For the item assessing if the task inspired participants to think pleasant thoughts (Item 4), the ANOVA demonstrated a significant main effect for Event, $F(1$, $56)=.4 .11, p=.05$. Participants in the Smile-Happy $(M=2.93, S D=1.49)$ and No-smile-Happy groups $(M=2.93, S D=1.62)$ rated this item higher compared to participants in the SmileNeutral $(M=2.14, S D=1.29)$ and Neutral groups $(M=2.29, S D=.99)$. There were no significant main effects for Smile, $F(1,56)=.05, p=.83$, or interaction between Smile and Event, $F(1,56)=.04, p=.84$, for this item.

Thinking about Plans after the Study. There was a significant Smile by Event interaction effect for the item assessing if participants were thinking about their plans for after the study (Item 6), $F(1,57)=4.05, p=.05$. Simple main effects demonstrated a significant difference between the smile and no-smile groups in the personally-relevant photograph conditions, $F(1$, $57)=4.20 p=.05$, with the No-smile Happy group $(M=3.07, S D=1.44)$ demonstrating more thoughts about what they were going to do after the study was completed than those in the Smile-Happy group $(M=2.00, S D=1.04)$ (see Table 17). There was no significant difference 
between the smile and no-smile groups in the neutral photograph conditions, $F(1,57)=.59, p=$ .45 . The Smile-Neutral group $(M=2.73, S D=1.62)$ and the Neutral group $(M=2.35, S D=1.41)$ demonstrated a relatively similar level of distraction by thoughts of plans after the study. There was no significant main effects for Smile, $F(1,57)=.91, p=.34$, or Event, $F(1,57)=.001, p=$ .98.

Anticipating a Long Task. There was also a significant Smile by Event interaction effect for item $8, F(1,57)=4.42, p=.04$, thinking that the stressor task was going on for a long time. Simple main effects demonstrated a significant difference between the smile and no-smile groups for the personally-relevant photograph condition, $F(1,57)=5.32, p=.03$, with the No-smile Happy group $(M=3.80, S D=1.94)$ thinking the study was going on too long more than those in the Smile-Happy group $(M=2.43, S D=1.34)$ (see Table 17$)$. There were no significant differences between the smile and no-smile groups for the neutral photograph condition, $F(1,57)$ $=.40, p=.53$, with the Smile-Neutral group $(M=2.53, S D=1.85)$ and the Neutral group $(M=$ $2.18, S D=1.19)$ demonstrating a relatively similar level of distraction by anticipating a long stressor task. There was no significant main effects for Smile, $F(1,57)=1.52, p=.22$, or Event, $F(1,57)=3.42, p=.07$. There were no significant effects for the other five items of this part of the post-experimental questionnaire (i.e., items 1, 2, 3, 5, and 7).

Ratings of Pleasant Event Photos. To further examine the specific effects of the recovery tasks on positive emotions, the ratings of the personally-relevant photographs were examined by comparing the responses of participants in the two groups that viewed personally-relevant photographs (see Table 18). Participants in the Happy-Smile and No-Smile-Happy Groups rated how happy they felt when the event in the photographs initially occurred. The personallyrelevant pictures were rated on a Likert-type scale with anchors of $1=$ Not at all happy to $9=$ 
Extremely happy. An independent samples t-test revealed no significant difference between the Smile-Happy group $(M=7.79, S D=1.63)$ and the No-smile-Happy group $(M=7.40, S D=$ $2.50), t(27)=.49, p=.63$. These results indicate that participants in both personally-relevant photograph groups selected photographs that were associated with a medium to a high level of happiness.

Ratings of Neutral Photos. The other two groups (Smile-Neutral and Neutral groups) rated how neutral they viewed the IAPS photographs (see Table 18). The non-personallyrelevant photographs were rated on a Likert-type scale with anchors of $1=$ Not at all neutral to 9 $=$ Extremely neutral. An independent samples t-test revealed no significant difference between the Smile-Neutral group $(M=7.93, S D=1.33)$ and the Neutral group $(M=8.35, S D=1.06)$, $t(29)=-.99, p=.33$. These results indicate that participants in both neutral photograph groups rated the IAPS images as neutral.

Purpose of the Study. Finally, a manipulation check was conducted to determine if participants were able to guess the actual purpose of the facial muscle manipulation via chopstick placement. A chi-square test of independence revealed no significant group differences on their ability to detect the purpose of the study, $X^{2}(6)=4.90, p=.56$ (see Table 19).

\section{Discussion}

The purpose of this study was to examine the psychophysiological effects of positive emotions, specifically how positive emotions affected cardiovascular recovery from a mental arithmetic stressor task. In this study, positive emotions were induced through two main methods, through the manipulation of facial muscles and exposure to personally-relevant photographs of a positive event. The results of the present study found no effects of positive emotions on any measure of cardiovascular functioning during recovery from stress. These 
results are largely inconsistent with other research demonstrating that positive emotions improve physiological recovery from stress (Fredrickson \& Levenson, 1998; Kraft \& Pressman, 2012). Previous research demonstrated that exposure to happy films sped physiological recovery from stress (Fredrickson \& Levenson, 1998). Further, other research found that a facial portrayal of the Duchenne smile resulted in lower heart rates during a recovery period as compared to a neutral group (Kraft \& Pressman, 2012). However, other research has failed to demonstrate that the induction of positive emotions reliably improves physiological recovery (Deiss Jr., 2012; Hannesdottir, 2007; Purdum, 2010). Findings from the current investigation conform to this latter group of studies that have failed to find support for the undoing hypothesis.

One potential explanation for the inconsistency of results across studies of this nature is the type of task used to induce positive emotions. In the current study the facial portrayal of the Duchenne smile was employed as one method to induce positive emotions. Research has demonstrated that activation of specific facial muscles associated with a genuine or Duchenne smile induces positive emotions (R. J. Davidson, 1992). Further, more recent research examined how the manipulation of these facial muscles would affect physiological recovery and found that smiling resulted in lower heart rates following stressor tasks (Kraft \& Pressman, 2012). There are several differences between the study conducted by Kraft and Pressman and the present study that may account for the inconsistent findings. In the Kraft and Pressman study, participants engaged in two stressor tasks (a star-tracing task and a cold pressor task) for a total of three minutes. During the stressor tasks, participants engaged in the manipulation of facial muscles in either smiling or neutral poses. In contrast, the present study employed a mental arithmetic stressor task during which participants engaged in the same facial muscle manipulation. Unlike the Kraft and Pressman task, participants mimicked a genuine smile for five minutes during the 
present study. The difference in duration of mimicking a genuine smile could explain the difference in results. In the present study, participants rated the level of difficulty of maintaining a genuine smile, how tired they felt when engaging in the facial feedback task, and how uncomfortable they felt when engaging in the facial muscle manipulation. Results of the selfreport data indicated that participants described the task as having a medium level of difficulty, resulting in a medium level of tiredness, and resulting in a medium level of discomfort. Further, during the stressor period, the Smile-Happy and Smile-Neutral groups demonstrated greater DBP reactivity compared to the No-smile groups. This greater DBP reactivity demonstrated by the Smile groups provides further evidence that the smile manipulation may have contributed to rather than reduced stress. It is possible that the five minute duration of the facial feedback was too long and instead of improving physiological recovery, the facial muscle manipulation resulted in an additional level of stress and/or discomfort. Another difference between the Kraft and Pressman and the current studies pertains to awareness of the purpose of the study. Kraft and Pressmen found that participants who were instructed to smile and were given the chopsticks to assist in this task exhibited the lowest HRs. In contrast, the purpose of the facial manipulation in the current study was not shared directly with participants at the beginning of the experiment. In this regard, the facial muscle manipulation may only be effective when participants are specifically instructed to smile to the best of their ability during the task.

In addition, the other task employed in the current study, recalling a happy memory through use of personally-relevant photographs, has not been examined previously and therefore, it was unknown how this task would affect physiological recovery. Other research has employed external tasks (e.g., exposure to a happy film) and demonstrated that exposure to films of this nature resulted in faster physiological recovery from stress (Fredrickson \& Levenson, 1998). 
Further, in other studies, participants recalled a happy memory; however, research using these methods have failed to demonstrate an effect on physiological recovery (Hannesdottir, 2007). In the present study, it was hypothesized that the use of personally-relevant photographs to prompt the recall of a happy memory would serve as an external task similar to the use of happy film clips and would therefore, improve physiological recovery from stress. It is possible that the personally-relevant photographs did not serve as a sufficient stimulus to prompt recall and that physiological recovery was unaffected as a result. It is also possible that participants did not engage in the recall of the memory associated with the personally-relevant photographs. Although the Smile-Happy and No-Smile-Happy groups rated the personally-relevant photographs as associated with medium to a high level of feelings of happiness at the time of the event, it is not possible to assess how successful participants were at engaging in the recall of the happy memory. Further, in other research studies employing external tasks, participants are typically exposed to the external tasks (e.g., happy films) following the stressor task. However, in the present study participants were exposed to the personally-relevant photographs and engaged in the recall of the memory during the stressor task. It is possible that participants experienced difficulty recalling the memory associated with the photographs as they were required to alternate between paying attention to the mental arithmetic problems and the recall task. This is supported by the finding of greater SBP reactivity observed among participants in both personally-relevant photograph groups. Research comparing recall of a happy memory alone with exposure to photographs that prompt recall of a happy memory to determine if the methods affect physiological recovery differently has yet to be conducted.

In addition to the failure to demonstrate any effect of PA induction on physiological recovery from stress discussed above, we were not able to provide evidence that either the recall 
or facial muscle manipulation strategies used in the current study induced positive emotions. Measures of PA revealed that scores declined from the initial resting period to the stress period for participants in all groups, including the control group, which indicates that the neither method of inducing positive affect was particularly successful. However, as discussed above, the reduction in PA that occurred during the stress period may have been due to the challenge of balancing both performance on mental arithmetic task and paying attention to the positive emotion-inducing strategy. This possibility is supported by the finding that participants in the personally-relevant photograph groups rated the photographs as more distracting than participants in the neutral photograph groups. If participants viewed one of the positive-emotion inducing tasks as distracting, this might contribute to difficulty balancing performance on both the stressor and PA-inducing tasks. Further, both of the positive-emotion induction tasks used in the current study resulted in greater BP reactivity (i.e., greater DBP reactivity for the smile manipulation, and greater SBP reactivity for viewing positive photographs). The greater reactivity along with the decreases in PA during the stressor and recovery period, suggests that the positive-emotion induction tasks were unsuccessful at inducing positive emotions and may have even contributed to increased stress. It is not possible to determine why these positiveemotion induction tasks failed to induce positive emotions and rather may have contributed to stress. However, it is possible that the effort of engaging in the mental arithmetic stressor task and the positive-emotion induction tasks may have contributed to increased stress as opposed to increased positive emotions. Alternatively, this could suggest that the positive mood induction tasks used in the present study evoked a level of PA, but that it was insufficient for countering the effects of the mental arithmetic stressor task. 
It is also possible that exposure to PA via recollection of positive memories results in increased cardiovascular arousal, but that this increase is not related to stress, but rather to the elicitation of positive mood states. Indeed, the increased SBP observed during the stressful task among participants viewing photographs of happy events could reflect an "excitement" response rather than a stress response, a hypothesis previously considered by researchers in the field (Hannesdottir, 2007; Yogo, Hama, Yogo, \& Matsuyama, 1995). In Hannesdottir's study, the group that recalled a happy memory demonstrated the highest mean HR during recovery, although it failed to reach statistical significance. In the current study, a comparable finding was observed in that groups viewing the photographs of pleasant events tended to show slower rates of SBP recovery $(p<.06)$ following completion of the stressful task. In this case, exposure to PA may result in an active coping task and consequently lead to increased cardiovascular activity in contrast to passive emotional elicitation methods that result in little or no cardiovascular change (Fredrickson et al., 2000; Pressman \& Cohen, 2005).

Another potential explanation for the difference in results between the present study and previous research is the type of stressor tasks employed. In the present study, a mental arithmetic task was employed. Only one other study in the literature on the undoing effect has employed a mental arithmetic stressor task, and this prior study found no effect of positive emotions on physiological recovery (Kaczmarek, 2009). Other studies that found an effect of positive emotions on physiological recovery have employed other stressor tasks. For example, Fredrickson and Levenson (1998) employed a fear film and a sad film as eliciting stimuli. A variety of other stressor tasks have been employed in the literature, including speech tasks (Dowd et al., 2010; Fredrickson et al., 2000; Steptoe et al., 2007; Tugade \& Fredrickson, 2004), star tracer and cold pressor tasks (Kraft \& Pressman, 2012), anticipation of a stressful event 
[answering a statistical question (Papousek et al., 2010)], and exposure to disgust-eliciting images from the IAPS (Sokhadze, 2007). Research suggests that a variety of stressor tasks induce subjective feelings of stress; however, a majority of stressor tasks used in the literature do not reliably activate the sympathetic nervous system (Fechir et al., 2008).

Further, research suggests that physiological responses to stressor tasks may vary depending on the type of task, active vs. passive. Active-coping tasks, such as the mental arithmetic task or a social stress task, result in a different pattern of physiological responding as compared to a passive-coping task, such as a cold pressor task or watching film clips. Activecoping tasks typically produce increases in blood pressure as a result of increased cardiac output and a decrease in vascular resistance (Sherwood, Dolan, \& Light, 1990). In contrast, passivecoping tasks produce increases in blood pressure through an increase in vascular resistance and a smaller increase in cardiac output. In the literature examining the undoing hypothesis, a variety of active- and passive-coping stressor tasks have been employed. Overall, there is no consistent pattern of results based upon the type of stressor task employed. The research suggests that the effects of positive emotions on recovery from stressors may be dependent upon the type of stressor task and reactivity evoked. For example, recovery from a social stressor task may be improved by positive emotion induction tasks involving a social component as compared to a passive-emotion induction task, such as watching a positive-film clip. This is supported by evidence that different stressor tasks produce different patterns of physiological responding.

One study examined the pattern of sympathetic nervous system responses to several types of stressor tasks, including presentation of negative and positive affective pictures, the colorword interference test (CWT), mental arithmetic test, speech task, and a singing aloud task (Fechir et al., 2008). The results indicated that only the CWT reliably activated the sympathetic 
nervous system and induced high ratings of subjective stress. Importantly, the study found that although the mental arithmetic task was rated as the most stressful subjectively, heart rate and skin conductance were the only measures of sympathetic nervous system activity that increased. The authors concluded that the CWT should be considered the recommended stressor task when researchers are examining measures of sympathetic nervous system activity. Future research should examine how the different types of stressor tasks and duration of engagement in the stressor tasks affects physiological recovery. Given the lack of attention to this issue in the literature, however, we are not certain whether the positive mood effects that have been observed previously are mediated by the sympathetic nervous system. In fact, it is entirely possible that these effects are mediated by parasympathetic activation.

It is also important to consider the lack of HR and HRV reactivity to the mental arithmetic stressor task observed in the present study. The mental arithmetic stressor task used in the current study only elicited SBP and DBP reactivity, which limited the ability to examine recovery using other physiological measures. There are several potential explanations for the lack of reactivity in HR and HRV observed in this study. First, participants' performance on the mental arithmetic stressor task was relatively poor. In previous research, Salvia et al. (2013) found a mean rate of $15.8 \%$ successful trials for difficult problems and a mean rate of $75.5 \%$ successful trials for easy problems. Participants in Salvia et al.'s study received information regarding reaction time, response accuracy, and general score following each math problem. The researchers hypothesized that this information would help maintain participants' motivation. In the present study, participants did not receive information regarding their performance after each math problem. Therefore, it is possible that participants became discouraged and were less motivated to exert any effort in answering the math problems. This lack of effort could have 
reduced the magnitude of physiological reactivity typically seen in response to mental arithmetic stressor tasks. Although participants were instructed to try their best on the math problems, it is possible that participants failed to follow these instructions. Further, because participants were engaged in multiple tasks simultaneously (i.e., mental arithmetic, holding the smile or no-smile manipulation, viewing personally-relevant photographs or neutral photographs), it may have limited participants' ability to attend to the mental arithmetic task fully. However, because this study did not include a condition that involved only completing the mental arithmetic task and previous studies did not report the magnitude of HR change that occurred among study participants (Kraft \& Pressman, 2012; Salvia et al., 2013), it is not possible to further evaluate this explanation.

The data analytic approach towards examining recovery from stress is also important to consider in making comparisons among studies that comprise this literature. In the present study, AUC was the primary strategy used to examine physiological recovery. This approach has not previously been used in the literature examining the effects of positive emotions on physiological recovery. Rather, a majority of studies examined the duration of time it took for participants to return to baseline (Fredrickson \& Levenson, 1998; Fredrickson et al., 2000; Hannesdottir, 2007; Tugade \& Fredrickson, 2004). Critically, three of the studies that defined recovery as return to baseline demonstrated support for the undoing hypothesis (Fredrickson \& Levenson, 1998; Fredrickson et al., 2000; Tugade \& Fredrickson, 2004). In the present study, SBPs recovered to pre-task levels and remained below the average resting levels by minute 6 of the recovery period. This differs greatly from previous research that demonstrated a return to baseline as quick as 35.9 seconds (Fredrickson \& Levenson, 1998, Study 2). For purposes of comparing the results of the current study with prior work, it is even more problematic for DBP, because, on average, 
DBP never returned to pre-task levels for most participants during the entire recovery period in the current study. As discussed in the introduction, there have been several definitions of recovery, including time to recovery and AUC. Although time to return to baseline has been associated with the undoing effect, research has demonstrated that time to recovery is an unreliable method for analyzing recovery (Christenfeld et al., 2000). Furthermore, times to recover to resting levels of SBP and DBP were not influenced by either PA induction method examined in the current study. As note in the introduction, research has demonstrated that the AUC method is a more reliable method for analyzing recovery (Linden et al., 1997). Because several studies that demonstrated support for the undoing hypothesis defined recovery as time to recovery and it is not a particularly reliable measure of recovery, support for the undoing hypothesis may not be as strong as initial reports appeared to indicate.

It is possible that the different approaches for examining recovery could contribute to inconsistent findings. Further, the typical length of recovery observed in several studies was $30 \mathrm{~s}$ post-task (Fredrickson \& Levenson, 1998; Tugade \& Fredrickson, 2004). In the current study, there was delay between the end of the stressor period and the start of the recovery period during which participants completed the PANAS-X. Physiological measures were not recorded during this time and therefore, it is possible that participants could have experienced significant physiological recovery during the delay between the end of the stressor period and the start of the recovery period. Indeed, this is supported by evidence that participants in the current study demonstrated substantial recovery from the last measure during the stressor task and the start of the recovery period. For example, the last minute of SBP during the stressor period was 118.5 $\mathrm{mg} \mathrm{Hg}(S D=11.41)$ for all participants, $6 \mathrm{~mm} \mathrm{Hg}$ above the mean resting SBP of $112.5 \mathrm{~mm} \mathrm{Hg}$ $(S D=9.73)$. The first reading of SBP during the recovery period was $114.7(S D=11.41)$, 
revealing that over $67 \%$ of the SBP response had already recovered prior to taking the first SBP reading during the recovery period. Similarly, the last minute of DBP during the stressor period was $73.1 \mathrm{mg} \mathrm{Hg}(S D=8.36)$, showing an $8.1 \mathrm{~mm} \mathrm{Hg}$ increase from the mean resting DBP of $65.0 \mathrm{~mm} \mathrm{Hg}(S D=9.40)$. The first reading of DBP during the recovery period was $66.8(S D=$ 9.39). Like SBP, this reveals that $77.8 \%$ of the DBP reactivity to the task was eliminated before the first DBP measure was obtained during the recovery period. Cumulatively, this provides support that the lack of recovery observed during the recovery period could have occurred in part because of the delay between the end of the stressor period and the start of the recovery period.

One final explanation to consider when evaluating the results of the current study was the design of the study. In the current study, participants engaged in the positive emotion induction tasks during the stressor tasks. The study was designed in this manner in part to replicate the study by Kraft and Pressman (2012). In the Kraft and Pressman study, participants engaged in the facial muscle manipulation during the stressor tasks. In contrast, in other studies, participants engaged in positive emotion induction tasks during the recovery period (Fredrickson \& Levenson, 1998; Tugade \& Fredrickson, 2004). In the current study, participants engaged in the facial muscle manipulation and recall of a personally-relevant memory during the stressor period. Indeed, SBP demonstrated a small habituation effect (approximately $2 \mathrm{~mm} \mathrm{Hg}$ ) during the stressor period, which suggests that participants experienced some physiological recovery during the stressor period. However, there was no habituation effect observed for DBP or HR during the task period. Further, participants demonstrated a large percentage of recovery between the stressor and the first minute of the recovery period. Additional research is needed to uncover the optimal timing of exposure to positive emotions to determine whether they should occur concurrent to engagement in stress or following exposures to stress. 
Strengths and Limitations. It is important to consider the strengths and limitations of this study when evaluating the results. The design of the study, a between-subjects design, allowed for the examination of two methods of inducing positive emotions to determine if one method was superior to the other at inducing positive emotions and thereby resulted in improved cardiovascular recovery from stress. An additional major strength of the present study was the use of a multi-modal assessment of stress and positive emotions. In the present study, stress and positive emotions were assessed through use of subjective, physiological, and behavioral measures. The physiological measures enabled examination of both sympathetic and parasympathetic responses. Examination of both sympathetic and parasympathetic responses is a particular strength of the present study as other studies examining the effects of positive emotions on cardiovascular recovery have only examined physiological indicators that are influenced by both branches of the autonomic nervous system.

The present study also employed behavioral observations of the smile and no-smile task to ensure that participants engaged in the tasks throughout the stressor period. Additional subjective measures were used to assess the difficulty of the smile and no-smile tasks. A few previous studies (Fredrickson \& Levenson, 1998, Study 2; Kraft \& Pressman, 2012) used behavioral observations to determine adherence to the facial feedback hypothesis. Since the primary purpose of the behavioral observations was to serve as a manipulation check, the behavioral measure (i.e., smile or no-smile for 4 minutes during the stressor period) was quite simple. Other studies (Kraft \& Pressman, 2012) employed more sophisticated methods of behavioral observations, like the Facial Action Coding System (FACS). Although the behavioral observation system used in the present study was sufficient to determine if participants were adherent to the smile and no-smile tasks, this method was rather simple. A more sophisticated 
method such as the FACS would have enabled coders to determine if other facial muscles were activated and thereby determine if participants were experiencing other positive or negative emotions. That said, only moderate levels of inter-rater reliability were observed in the current study, suggesting that the portrayal of the Duchenne smile is not always easy to detect.

The use of AUC to examine physiological recovery is an additional strength to discuss. Previous studies have not employed consistent methods of analyzing physiological recovery. For example, several studies defined recovery as the time it took for participants' physiological levels to return to baseline levels (Fredrickson \& Levenson, 1998; Fredrickson et al., 2000; Hannesdottir, 2007; Tugade \& Fredrickson, 2004). This operationalization of recovery is problematic for several reasons. First, not all participants will return to baseline physiological levels. Second, this operationalization does not allow for the use of more advanced statistical techniques, such as curve-fitting estimates or AUC. The use of AUC is a particular strength of this study.

A final strength to discuss is the use of personally-relevant photographs to induce positive emotions. A majority of previous studies have used standardized images to induce positive emotions and one study prompted participants to recall a personal happy memory (Hannesdottir, 2007). In this study, participants who recalled a personal happy memory produced the highest subjective ratings of positive emotions. Therefore, the use of personally-relevant photographs of a positive event was an additional strength of the study.

Although there are multiple strengths of this study, it is also important to consider the limitations of the present study. First, after the end of the stressor period participants completed the PANAS-X. However, the recovery period did not begin until the PANAS-X was completed and therefore, participants' physiological recovery may have begun prior to the recovery period. 
As discussed previously, there is evidence of early physiological recovery, which limits the ability to draw conclusions about physiological recovery and limits the ability to determine if one method of inducing positive affect affected physiological recovery differently than the other.

An additional limitation to consider is the timing of the positive induction tasks. In the present study, participants engaged in the smile and personally-relevant photograph tasks during the presentation of the stressor stimuli. Therefore, it is possible that the physiological effects of the stressor task were reduced and that the effects of the recovery tasks were also reduced. Previous research examining the effects of positive emotions on recovery utilized a slightly different design in regard to the timing of the positive emotion induction tasks. In general, positive emotion induction tasks followed completion of a stressor task (Fredrickson \& Levenson, 1998). In contrast, in the current study, participants engaged in the stressor task and positive emotion induction tasks simultaneously. One previous study used a similar design in which half of the participants received a positive emotion or neutral mood induction prior to a stressful task and the other half of participants received the mood induction following the stressful task (Monfort, 2012). The study demonstrated no effect of positive emotions on buffering the effects of stress or in promoting physiological recovery. In the present study, the critical difference in timing of the positive emotion induction task may have prevented positive emotions from influencing physiological recovery.

In the present study, personally-relevant positive photographs were compared to nonpersonally-relevant neutral photographs. Consequently, the stimuli used in this study differed on two dimensions, emotional valence (positive vs. neutral) and relevancy of the photographs (personally-relevant vs. non-personally-relevant). In designing the current study, personallyrelevant neutral photographs (e.g., picture of one's kitchen chair) were not used as comparison 
stimuli. Likewise, photographs of non-personally relevant positive events (e.g., pictures of a stranger's birthday party) were alson not employed. Therefore, it is unknown whether the effects demonstrated in this study were due to the relevance or the valance of the photographs (i.e., positive versus neutral).

It is also important to note that there were no group differences in General PA or NA during the stressor period. Because positive emotion was induced in three of the four groups, it was expected that the Smile-Happy, Smile-Neutral, and the No-smile-Happy groups would have demonstrated higher ratings of general PA as compared to the Neutral group during the stressful task. This lack of induction of positive emotions is further supported by no group differences on the post experimental questionnaire on items that may reflect some exposure to PA. It is possible that PA induction did not occur due to a ceiling effect on the measure of PA employed. Although relatively high levels of PA were observed during the initial resting period $(M=23.33, S D=$ 6.47), they were not as high as levels observed in prior research on two samples of undergraduates rating PA in the moment $(M=29.0 ; 25.3, S D=8.0 ; 7.2$; Watson \& Clark, 1994). An additional limitation to consider is that the induction of broad PA states differs from the induction of targeted emotions (e.g., happiness, excitement, etc.) (DeSteno et al., 2013; Dockray \& Steptoe, 2010; Loewenstein \& Lerner, 2003; Keltner \& Lerner, 2010). This finding has been supported within the literature examining the undoing hypothesis. One study that failed to demonstrate support for the undoing effect proposed that the quality and intensity of physiological responses to positive emotions and stressors must be similar in order to promote physiological recovery (Kaczmarek, 2009).

Further, as discussed previously, the effort of balancing engagement in the mental arithmetic task and the positive-emotion induction tasks may have interfered with any positive 
effect of PA. This is supported by evidence in which participants viewed the positive-emotion induction tasks as effortful. Participants rated the positioning of the chopsticks during the task, regardless of Duchenne-smile manipulation or no-smile manipulation, as moderately difficult, causing a moderate level of tiredness, and causing a moderate level of discomfort. These ratings suggested that participants viewed the positioning of the chopsticks as effortful, which may have prevented it from inducing positive emotions. Additionally, participants in the personallyrelevant photograph groups rated the happy photographs as distracting, which further suggests that the positive-emotion induction tasks required additional effort, which likely limited its effectiveness of inducing PA and potentially reducing the magnitude of the stress response.

A previous study used the same facial muscle manipulation; however, participants only positioned the chopsticks in their mouth during a three-minute stress period (Kraft \& Pressman, 2012). In the current study, the stressor period lasted five minutes and participants were instructed to hold the position of the chopsticks during the entire length of the stressor task. Therefore, the length of the task in the present study may have been more effortful than previous research and may have contributed to muscle fatigue with the participants. Additionally, the Kappa coefficients in the present study suggest that it was difficult to measure adherence reliably to the Duchenne-smile and no-smile manipulation. There was moderate agreement for behavioral coding as demonstrated by the Kappa coefficients. This moderate agreement suggests that it was difficult for coders to determine if participants adhered to the designated position consistently for the full task period. This is particularly problematic for the Smile groups in which lower Kappas were observed. If the smile groups failed to maintain a true Duchenne-smile for the majority of the stressor task, then this task would be less likely to induce positive emotions. Future research 
should explore how differences in the timing of induction of positive emotions affects physiological recovery.

A final limitation in the present study was the use of a young adult sample of university undergraduate students, which significantly limits the generalizability of findings. There is limited research examining age differences in the undoing effect of positive emotions. However, a few studies demonstrated partial support for the undoing hypothesis among middle-aged and older adults (Ong \& Allaire, 2005; Steptoe et al., 2007; Yuan et al., 2010). Although limited, these findings are particularly interesting as other evidence indicates age differences in emotional well-being. The socioemotional selectivity theory proposes that emotional well-being improves as we age because older adults prioritize the important aspects of their life (Carstensen, Pasupathi, Mayr, \& Nesselroade, 2000). Research has demonstrated a positive relation between aging, emotional well-being, emotional stability, and emotional complexity (Carstensen et al., 2011). Further, this study found that the experience of positive emotions predicted reduced mortality over a period of 13 years. As discussed previously, PA and positive emotions are associated with improved physical health (Boehm \& Kubzansky, 2012; Gallo, Ghaed, \& Bracken, 2004). As positive emotions are associated with improved physical health and because older adults demonstrate improved emotional well-being, future research should further examine the undoing effect across the lifespan.

Future Directions and Conclusions. Research examining the undoing effects of positive emotions is mixed. Several studies have demonstrated how positive emotions facilitate physiological recovery from stress (Fredrickson \& Levenson, 1998; Kraft \& Pressman, 2012; Tugade et al., 2004); however, other studies suggested that exposure to positive emotions had no effect on physiological recovery (Deiss Jr., 2012; Hannesdottir, 2007). One potential explanation 
of the differences in findings is how recovery is operationalized and analyzed. For example, recovery is occasionally operationalized as the time that elapses before a participant's physiological levels return to baseline (Fredrickson \& Levenson, 1998). Other definitions of recovery include relative recovery to baseline, relative recovery to stressor, and recovery slope. In the present study, recovery was analyzed by examining AUC. Future research should emphasize a consistent operationalization and analysis of recovery. An additional future direction related to the operationalization of recovery is the type of physiological measures employed in the present study. The present study employed cardiovascular measures that examined both sympathetic and parasympathetic activity, which was a strength of the study. However, the use of only cardiovascular measures to examine recovery provides a narrow understanding of how positive emotions affect recovery, particularly if they influence physiological reactivity in ways that are not detected by the measures employed in this study.

An additional area of future research is to examine the effects of different affective states on physiological recovery. A majority of the literature on the undoing hypothesis has focused on the effects of happiness on physiological recovery from stress (Fredrickson \& Levenson, 1998; Hannesdottir, 2007; Kraft \& Pressman, 2012; Tugade \& Fredrickson, 2004). Previously research focused on the examination of broad emotional or valence states (e.g. negative vs. positive) (DeSteno et al., 2013) . However, recent research has demonstrated the importance of examining the effects of specific emotional states (e.g., happiness, gratitude) (Desteno et al., 2013; Loewenstein \& Lerner, 2003). Although specific emotional states (e.g., happiness) can be categorized as one type of broad affective state (e.g., positive affect), there is little evidence to assume that all specific emotional states within the same affective state would produce the same physiological response patterns (Desteno et al., 2013). In a recent conceptual article examining 
affective science and health, the authors argue that specific emotional states enable individuals to address distinct challenges and that as such it is likely that specific emotional states would differ in physiological response patterns (Desteno et al., 2013). To further explore the difference in physiological response patterns to a range of emotional states, it would be important to include multiple measures of physiological responding, including measures of sympathetic and parasympathetic nervous system activity as well as immune and endocrine activity. It is also important to consider how the type of stressor task may influence whether a positive emotion induction will promote physiological recovery. For example, exposure to photographs of a happy event may promote recovery from social stress, but may not promote recovery from a cognitive stressor like mental arithmetic.

A final area of future research is to examine the long-term effects of positive emotions on physiological recovery. For example, it may be possible that people who are more likely to use positive emotions demonstrate better physiological recovery from stress but this may not be observed immediately following exposure to a short laboratory stressor. Further, positive emotions may affect physiological recovery from stress in such a way that those who experience it regularly are less likely to develop future cardiovascular disease. This type of research may lead to examination of clinical interventions using positive emotions to reduce the effect of stress on future cardiovascular disease risk.

In conclusion, this study was designed to help expand the literature on the undoing hypothesis by comparing two methods of inducing positive emotions to determine how each method affected physiological recovery from stress. The recall of a happy memory through personally-relevant photographs was designed to bridge a gap in the literature by using photographs to help a recall of positive emotions. One possible reason that other recall tasks used 
in previous studies did not affect physiological recovery is that participants did not engage in the task fully (Hannesdottir, 2007). Therefore, the personally-relevant photographs were hypothesized to assist participants in recalling a happy memory. The other method of inducing positive emotions, the facial feedback task, was employed to replicate previous findings (Kraft \& Pressman, 2012). The current study found no effect of positive emotions on physiological recovery. As discussed previously, there are several possible reasons for the lack of findings in the current study. However, it is also possible that positive emotions may not reliably improve physiological recovery or that individual differences in personality, coping styles, and emotion regulation may interact with positive emotions to affect physiological recovery. The possibility that positive emotions do not reliably improve physiological recovery is supported by other research which has failed to support the undoing hypothesis (Deiss Jr., 2012; Hannesdottir, 2007; Kaczmarek, 2009; Monfort, 2012). The literature on the undoing hypothesis is mixed and future research will need to attempt further replications of previous studies which have supported the undoing effect to determine if positive emotions reliably improve physiological recovery from stress. 


\section{References}

Algoe, S. B., \& Stanton, A. L. (2012). Gratitude when it is needed most: Social functions of gratitude in women with metastatic breast cancer. Emotion (Washington, D.C.), 12(1), 1638. doi:10.1037/a0024024

Allen, M. T. (2000). Psychosocial Notebook. MacArthur: Research Network on SES \& Health. Retrieved from http://www.macses.ucsf.edu/research/psychosocial/reactivitytable.php

Allen, M. T., Boquet, a J., \& Shelley, K. S. (1991). Cluster analyses of cardiovascular responsivity to three laboratory stressors. Psychosomatic Medicine, 53(3), 272-88. Retrieved from http://www.ncbi.nlm.nih.gov/pubmed/1882009

Boehm, J. K., \& Kubzansky, L. D. (2012). The heart's content: The association between positive psychological well-being and cardiovascular health. Psychological Bulletin, 138(4), 65591. doi:10.1037/a0027448

Borghi, C., Costa, F. V., Boschi, S., Mussi, A., \& Ambrosioni, E. (1986). Predictors of stable hypertension in young boderline subjects: A five-year follow-up study. Jounral of Cardiovascular Pharmacology, 8 (Suppl., S138-S141.

Brandt, F. S., \& Cazzaniga, A. (2007). Hyaluronic Acid Fillers : Restylane and Perlane. Facial Plastics Surgery Clinics of North America, 15, 63-76. doi:10.1016/j.fsc.2006.11.002

Carrico, A. W., Johnson, M. O., Colfax, G. N., \& Moskowitz, J. T. (2010). Affective correlates of stimulant use and adherence to anti-retroviral therapy among HIV-positive methamphetamine users. AIDS and Behavior, 14(4), 769-77. doi:10.1007/s10461-0089513-y

Carstensen, L. L., Pasupathi, M., Mayr, U., \& Nesselroade, J. R. (2000). Emotional experience in 
everyday life across the adult life span. Journal of Personality and Social Psychology, 79(4), 644-55. Retrieved from http://www.ncbi.nlm.nih.gov/pubmed/11045744

Carstensen, L. L., Turan, B., Scheibe, S., Ram, N., Ersner-Hershfield, H., Samanez-Larkin, G. R., ... Nesselroade, J. R. (2011). Emotional experience improves with age: evidence based on over 10 years of experience sampling. Psychology and Aging, 26(1), 21-33. doi:10.1037/a0021285

Cavanagh, C. E. (2013). Emotion regulation and cardiovascular response to emotion provocation: reappraisal versus suppression. West Virginia University.

Chida, Y., \& Steptoe, A. (2010). Greater cardiovascular responses to laboratory mental stress are associated with poor subsequent cardiovascular risk status: A meta-analysis of prospective evidence. Hypertension, 55(4), 1026-32. doi:10.1161/HYPERTENSIONAHA.109.146621

Christenfeld, N., Glynn, L. M., \& Gerin, W. (2000). On the reliable assessment of cardiovascular recovery: An application of curve-fitting techniques.pdf. Psychophysiology, 37, 543-550.

Cohen, S., Doyle, W. J., Turner, R. B., Alper, C. M., \& Skoner, D. P. (2003). Emotional style and susceptibility to the common cold. Psychosomatic Medicine, 65(4), 652-657. doi:10.1097/01.PSY.0000077508.57784.DA

Cole, C. R., Blackstone, E. H., Pashkow, F. J., Snader, C. E., \& Lauer, M. S. (1999). Heart-rate recovery immediately after exercise as a predictor of mortality. The New England Journal of Medicine, 341(18), 1351-7. doi:10.1056/NEJM199910283411804

Crawford, J. R., \& Henry, J. D. (2004). The positive and negative affect schedule (PANAS): Construct validity, measurement properties and normative data in a large non-clinical sample. The British Journal of Clinical Psychology, 43(Pt 3), 245-65. 
doi:10.1348/0144665031752934

Davidson, R. J. (1992). Emotion and affective style: Hemispheric substrates. Psychological Science, 3(I), 39-43.

Davis, J. I., Senghas, A., \& Ochsner, K. N. (2009). How does facial feedback modulate emotional experience? Journal of Research in Personality, 43(5), 822-829. doi:10.1016/j.jrp.2009.06.005

Davis, J. I., Senghas, A., \& Ochsner, K. N. (2010). The effects of Botox injections on emotional experience. Emotion, 10(3), 433-440. doi:10.1037/a0018690.The

Deiss Jr., D. M. (2012). An Experimental Replication and Refinement of the Undoing Hypothesis of Positive Emotions. Unpublished Dissertation. Retrieved from Arizona State University.

DeSteno, D., Gross, J. J., \& Kubzansky, L. (2013). Affective science and health: The importance of emotion and emotion regulation. Health Psychology, 32(5), 474-86. doi: $10.1037 / \mathrm{a} 0030259$

Dockray, S. \& Steptoe, A. (2010). Positive affect and psychobiological processes. Neuroscience and Biobehavioral Reviews, 35(1), 69-75. doi:10.1016/j.neubiorev.2010.01.006

Dolly, J. O., \& Aoki, K. R. (2006). The structure and mode of action of different botulinum toxins. European Journal of Neurology, 13(4), 1-9.

Dowd, H., Zautra, A., \& Hogan, M. (2010). Emotion, stress, and cardiovascular response: An experimental test of models of positive and negative affect. International Journal of Behavioral Medicine, 17(3), 189-194. doi:10.1007/s12529-009-9063-3

Ekman, P., Levenson, R. W., \& Friesen, W. V. (1983). Autonomic nervous system activity 
distinguishes among emotions. Science, 221(4616), 1208-1210.

Everson, S. A., G.A., K., Goldberg, D. E., \& Salonen, J. T. (1996). Anticipatory blood pressure response to exercise predicts future high blood pressure in middle-aged men. Hyperntension, 27(5), 1059-1064.

Faul, F., Erdfelder, E., Buchner, A., \& Lang, A. G. (2009). Statistical power analyses using G*Power 3.1: Tests for correlation and regression analyses. Behavior Research Methods, 41, 1149-1160. doi:doi: 10.3758/BRM.41.4.1149

Fechir, M., Schlereth, T., Purat, T., Kritzmann, S., Geber, C., Eberle, T., ... Birklein, F. (2008). Patterns of sympathetic responses induced by different stress tasks. The Open Neurology Journal, 2, 25-31. doi:10.2174/1874205X00802010025

Field, A. (2009). Discovering statistics using SPSS (3rd ed.). London: SAGE Publications.

Fredrickson, B. L., \& Levenson, R. W. (1998). Positive emotions speed recovery from the cardiovascular sequelae of negative emotions. Cognition \& Emotion, 12(2), 191-220. doi:10.1080/026999398379718

Fredrickson, B. L., Mancuso, R. A., Branigan, C., \& Tugade, M. M. (2000). The undoing effect of positive emotions. Motivation and Emotion, 24(4), 237-258. doi:0146-7239/00/12000237

Friedberg, J. P., Suchday, S., \& Shelov, D. V. (2007). The impact of forgiveness on cardiovascular reactivity and recovery. International Journal of Psychophysiology, 65(2), 87-94. doi:10.1016/j.ijpsycho.2007.03.006

Gallo, L. C., Ghaed, S. G., \& Bracken, W. S. (2004). Emotions and cognitions in coronary heart 
disease: Risk, resilience, and social context. Cognitive Therapy and Research, 28(5), 669694. doi:10.1023/B:COTR.0000045571.11566.19

Goodie, J. L., Larkin, K. T., \& Schauss, S. (2000). Validation of the Polar Heart Rate Monitor for Assessing HeartRate During Physical and Mental Stress. Journal of Psychophysiology, 14(3), 159-164. doi:10.1027//0269-8803.14.3.159

Hannesdottir, K. (2007). Reduction of fear arousal in young adults with speech anxiety through elicitation of positive emotions. Retrieved from Virginia Polytechnic Institute and State University.

Harrison, L. K., Carroll, D., Burns, V. E., Corkill, A.R., Harrison, C. M., Ring, C., \& Drayson, M. (2000). Cardiovascular and secretory immunoglobulin A reactions to humorous, exciting, and didactic film presentations. Biological Psychology, 52(2), 113-26. Retrieved from http://www.ncbi.nlm.nih.gov/pubmed/10699351

Kaczmarek, L. (2009). Resiliency, stress appraisal, positive affect, and cardiovascular activity. Polish Psychological Bulletin, 40(1), 46-53. doi:10.2478/s10059-009-0007-1

Kamarck, T. W., Eranen, J., Jennings, J. R., Manuck, S. B., Everson, S. A., Kaplan, G. A., \& Salonen, J. T. (2000). Anticipatory blood pressure responses to exercise are associated with left ventricular mass in Finnish men: Kuopio Ischemic Heart Disease Risk Factor Study. Circulation, 102(12), 1394-1399. doi:10.1161/01.CIR.102.12.1394

Keillor, J. M., Barrett, A. M., Crucian, G. P., Kortenkamp, S., \& Heilman, K. M. (2002). Emotional experience and perception in the absence of facial feedback. Journal of the International Neuropsychological Society, 8, 130-135.

Keltner, D., \& Lerner, J. S. (2010). Emotion. In D. Gilbert, S. Fiske, \& G. Lindzey (Eds.), The 
handbook of social psychology (5th ed., pp. 317-352). New York, NY: McGraw-Hill.

Kraft, T. L., \& Pressman, S. D. (2012). Grin and bear it: The influence of manipulated facial expression on the stress response. Psychological Science, 23(11), 1372-8. doi:10.1177/0956797612445312

Kubzansky, L. D., \& Kawachi, I. (2000). Going to the heart of the matter: Do negative emotions cause coronary heart disease? Journal of Psychosomatic Research, 48(4-5), 323-337. Retrieved from http://www.ncbi.nlm.nih.gov/pubmed/10880655

Lang, P. J., Bradley, M. M., \& Cuthbert, B. N. (1995). International Affective Picutre System (IAPS): Technical manual and affective ratings. University of Florida, Gainesville, FL.

Lang, P. J., Bradley, M. M., \& Cuthbert, B. N. (2008). International affective picture system (IAPS): Affective ratings of pictures and instruction manual. Technical Report A-8. University of Florida, Gainesville, FL.

Levenson, R. W., \& Ekman, P. (2002). Difficulty does not account for emotion-specific heart rate changes in the directed facial action task. Psychophysiology, 39(3), 397-405. doi:10.1017.S0048577201393150

Libkuman, T. M., Otani, H., Kern, R., Viger, S. G., \& Novak, N. (2007). Multidimensional normative ratings for the International Affective Picture System. Behavior Research Methods, 39(2), 326-34. Retrieved from http://www.ncbi.nlm.nih.gov/pubmed/17695361

Linden, W., Earle, T. L., Gerin, W., \& Christenfeld, N. (1997). Physiological stress reactivity and recovery: Conceputal siblings separated at birth? Journal of Psychosomatic Research, 42(2), 117-135. 
Loewenstein, G., \& Lerner, J. S. (2003). The role of affect in decision making. In K. Davidson, R. Goldsmith, H. \& Scherer (Ed.), Handbook of affective science (pp. 619-642). New York, NY: Oxford University Press.

Loewenstein, G., \& Lerner, J. S. (2003). The role of affect in decision making. In R. Davidson, H. Goldsmith, \& K. Scherer (Eds.), Handbook of affective science (pp. 619-642). New York, NY: Oxford University Press.

Marler, M. R., Jacob, R. G., Lehoczky, J. P., \& Shapiro, A. P. (1988). The statistical analysis of treatment effects ion 24-hour ambulatory blood pressure recordings. Statistics in Medicine, 7, 697-716.

Monfort, S. S. (2012). Anticipiatory positive emotion and recovery from stress. (Master's Thesis). Retrieved from ProQuest, Wake Forest University, Winston-Salem, NC.

Neumann, S. A., Waldstein, S. R., Sellers, J. J., Thayer, J. F., \& Sorkin, J. D. (2004). Hostility and distraction have differential influences on cardiovascular recovery from anger recall in women. Health Psychology, 23(6), 631-40. doi:10.1037/0278-6133.23.6.631

Newman, M. G., \& Stone, A. A. (1996). Does humor moderate the effects of experimentallyinduced stress? Annals of Behavioral Medicine, 18(2), 101-109. doi:10.1007/BF02909582

Niskanen, J.-P., Tarvainen, M. P., Ranta-Aho, P. O., \& Karjalainen, P. A. (2004). Software for advanced HRV analysis. Computer Methods and ProgrNiskanen, J.-P., Tarvainen, M. P., Ranta-Aho, P. O., \& Karjalainen, P. A. (2004). Software for Advanced HRV Analysis. Computer Methods and Programs in Biomedicine, 76(1), 73-81. doi:10.1016/j.cmpb.2004.03.004ams in Biomedicine, 76(1), 73-81. doi:10.1016/j.cmpb.2004.03.004 
Obrist, P. A. (1976). Presidential Address, 1975: The cardiovascular-behavioral interaction - As it appears today. Psychophysiology, 13(2), 95-107.

Ong, A. D., \& Allaire, J. C. (2005). Cardiovascular intraindividual variability in later life: The influence of social connectedness and positive emotions. Psychology and Aging, 20(3), 476-85. doi:10.1037/0882-7974.20.3.476

Papousek, I., Nauschnegg, K., Paechter, M., Lackner, H. K., Goswami, N., \& Schulter, G. (2010). Trait and state positive affect and cardiovascular recovery from experimental academic stress. Biological Psychology, 83(2), 108-115.

doi:10.1016/j.biopsycho.2009.11.008

Pieper, S., \& Brosschot, J. F. (2005). Prolonged stress-related cardiovascular activation: Is there any? Annals of Behavioral Medicine, 30(2), 91-103. doi:10.1207/s15324796abm3002_1

Prentice, P. (2009). Dispositional mindfulness and cardiovascular reactivity to sensory intake and sensory rejection tasks. Retrieved from West Virginia University.

Purdum, M. B. (2010). The effects of positive emotion, negative emotion, flourishing, and languishing on cardiovascular risk. Unpublished Dissertation. Retrieved from University of North Texas.

Roest, A. M., Martens, E. J., de Jonge, P., \& Denollet, J. (2010). Anxiety and risk of incident coronary heart disease: A meta-analysis. Journal of the American College of Cardiology, 56(1), 38-46. doi:10.1016/j.jacc.2010.03.034

Salvia, E., Guillot, A., \& Collet, C. (2013). The effects of mental arithmetic strain on behavioral and physiological responses. Journal of Psychophysiology, 27(4), 173-184. doi:10.1027/0269-8803/a000102 
Schwartz, A. R., Gerin, W., Davidson, K., Pickering, T. G., Brosschot, J. F., Thayer, J. F., ... Linden, W. (2003). Toward a causal nodel of cardiovascular responses to stress and the development of cardiovascular disease. Psychosomatic Medicine, 65(1), 22-35. doi:10.1097/01.PSY.0000046075.79922.61

Selye, H. (1973). The evolution of the stress concept: The originator of the concept traces its development from the discovery in 1936 of the alarm reaction to modern therapeutic applications of syntoxic and catatoxic hormones. American Scientist, 61(6), 692-699.

Sherwood, A., Dolan, C. A., \& Light, K. C. (1990). Hemodynamics of blood pressure responses during active and passive coping. Psychophysiology, 27(6), 656-68. Retrieved from http://www.ncbi.nlm.nih.gov/pubmed/2100351

Sokhadze, E. M. (2007). Effects of music on the recovery of autonomic and electrocortical activity after stress induced by aversive visual stimuli. Applied Psychophysiology and Biofeedback, 32(1), 31-50. doi:10.1007/s10484-007-9033-y

Soussignan, R. (2002). Duchenne smile, emotional experience, and autonomic reactivity: A test of the facial feedback hypothesis. Emotion, 2(1), 52-74. doi:10.1037//1528-3542.2.1.52

Stauber, S., Schmid, J.-P., Saner, H., Znoj, H., Saner, G., Grolimund, J., \& von Känel, R. (2013). Health-related quality of life is associated with positive affect in patients with coronary heart disease entering cardiac rehabilitation. Journal of Clinical Psychology in Medical Settings, 20(1), 79-87. doi:10.1007/s10880-012-9311-6

Steptoe, A., Gibson, E. L., Hamer, M., \& Wardle, J. (2007). Neuroendocrine and cardiovascular correlates of positive affect measured by ecological momentary assessment and by questionnaire. Psychoneuroendocrinology, 32(1), 56-64. 
doi:10.1016/j.psyneuen.2006.10.001

Stewart, J. C., \& France, C. R. (2001). Cardiovascular recovery from stress predicts longitudinal changes in blood pressure. Biological Psychology, 58(2), 105-20. Retrieved from http://www.ncbi.nlm.nih.gov/pubmed/11600240

Strack, F., Martin, L. L., \& Stepper, S. (1988). Inhibiting and facilitating conditions of the human smile: A nonobtrusive test of the facial feedback hypothesis. Journal of Personality and Social Psychology, 54(5), 768-77. Retrieved from http://www.ncbi.nlm.nih.gov/pubmed/3379579

Tabachnick, B. G., \& Fidell, L. S. (2013). Using multivariate statistics (6th ed.). Upper Sadle River, NJ: Pearson.

Tarvainen, M. P., \& Niskanen, J. (2008). Kubios HRV version 2.0: User's guide.

Tourangeau, R., \& Ellsworth, P. C. (1979). The role of facial response in the experience of emotion. Journal of Personality and Social Psychology, 37(9), 1519-31. Retrieved from http://www.ncbi.nlm.nih.gov/pubmed/501520

Treiber, F. A., Musante, L., Kapuku, G., Davis, C., Litaker, M., \& Davis, H. (2001). Cardiovascular $(\mathrm{CV})$ responsivity and recovery to acute stress and future $\mathrm{CV}$ functioning in youth with family histories of CV disease: A 4-year longitudinal study. International Journal of Psychophysiology, 41(1), 65-74. Retrieved from http://www.ncbi.nlm.nih.gov/pubmed/11239698

Tugade, M. M., \& Fredrickson, B. L. (2004). Resilient individuals use positive emotions to bounce back from negative emotional experiences. Journal of Personality and Social Psychology, 86(2), 320-33. doi:10.1037/0022-3514.86.2.320 
Tugade, M. M., Fredrickson, B. L., \& Barrett, L. F. (2004). Psychological resilience and positive emotional granularity: Examining the benefits of positive emotions on coping and health. Journal of Personality, 72(6), 1161-90. doi:10.1111/j.1467-6494.2004.00294.x

van Melle, J. P., de Jonge, P., Spijkerman, T. A., Tijssen, J. G. P., Ormel, J., van Veldhuisen, D. J., ... van den Berg, M. P. (2004). Prognostic association of depression following myocardial infarction with mortality and cardiovascular events: A meta-analysis. Psychosomatic Medicine, 66(6), 814-22. doi:10.1097/01.psy.0000146294.82810.9c

Watson, D., Clark, L. a, \& Tellegen, A. (1988). Development and validation of brief measures of positive and negative affect: The PANAS scales. Journal of Personality and Social Psychology, 54(6), 1063-70. Retrieved from http://www.ncbi.nlm.nih.gov/pubmed/3397865

Watson, D., \& Clark, L. A. (1992). Affects separable and inseparable: On the hierarchical arrangement of the negative affects. Journal of Personality and Social Psychology, 62(3), 489-505.

Watson, D., \& Clark, L. A. (1994). The PANAS-X manual for the Positive and Negative Affect Schedule - Expanded Form. Retrieved from http://www2.psychology.uiowa.edu/faculty/clark/panas-x.pdf

Watson, D., \& Pennebaker, J. W. (1989). Health complaints, stress, and distress: Exploring the central role of negative affectivity. Psychological Review, 96(2), 234-54. Retrieved from http://www.ncbi.nlm.nih.gov/pubmed/2710874

World Health Organization. (2011). Global atlas on cardiovascular disease prevention and control. World Health Organization. Retrieved February 22, 2016 from http://www.who.int/cardiovascular_diseases/publications/atlas_cvd/en/ 
World Health Organization. (2015). Cardiovascular diseases (CDs) Fact Sheet. World Health Organization. Retrieved February 22, 2016, from http://www.who.int/mediacentre/factsheets/fs317/en/

Wulsin, L. R. (2003). Do depressive symptoms increase the risk for the onset of coronary disease? A systematic quantitative review. Psychosomatic Medicine, 65(2), 201-210. doi:10.1097/01.PSY.0000058371.50240.E3

Yogo, Y., Hama, H., Yogo, M., \& Matsuyama, Y. (1995). A study of physiological response during emotional imaging. Perceptual and Motor Skills, 81(43-49).

Yuan, J. W., McCarthy, M., Holley, S. R., \& Levenson, R. W. (2010). Physiological downregulation and positive emotion in marital interaction. Emotion, 10(4), 467-474. doi:10.1037/a0018699

Yusuf, S., Reddy, S., Ounpuu, S., \& Anand, S. (2001a). Global burden of cardiovascular diseases: Part I: General considerations, the epidemiologic transition, risk factors, and impact of urbanization. Circulation, 104(22), 2746-2753. doi:10.1161/hc4601.099487

Yusuf, S., Reddy, S., Ounpuu, S., \& Anand, S. (2001b). Global burden of cardiovascular diseases: Part II: Variations in cardiovascular disease by specific ethnic groups and geographic regions and prevention strategies. Circulation, 104(23), 2855-2864. doi:10.1161/hc4701.099488 
Table 1

Demographic characteristics of the SONA (screening) sample, pilot sample, and the lab sample

\begin{tabular}{|c|c|c|c|c|c|c|}
\hline & \multicolumn{2}{|c|}{$\begin{array}{l}\text { Screening Sample } \\
\quad(\mathrm{n}=804)\end{array}$} & \multicolumn{2}{|c|}{$\begin{array}{l}\text { Pilot Sample } \\
\quad(\mathrm{n}=12)\end{array}$} & \multicolumn{2}{|c|}{$\begin{array}{l}\text { Lab Completers } \\
\quad(\mathrm{n}=61)\end{array}$} \\
\hline & Mean & $S D$ & Mean & $S D$ & Mean & $S D$ \\
\hline Age & 19.5 & 3.1 & 19.3 & .78 & 19.4 & 1.4 \\
\hline \multirow[t]{2}{*}{ BMI* } & -- & -- & -- & -- & 25.47 & 5.31 \\
\hline & $N$ & $\%$ & & & $n$ & $\%$ \\
\hline \multicolumn{7}{|l|}{ Sex } \\
\hline Male & 205 & 25.6 & 3 & 25.0 & 14 & 23.0 \\
\hline Female & 599 & 74.4 & 9 & 75.0 & 47 & 77.0 \\
\hline \multicolumn{7}{|l|}{ Race } \\
\hline White & 694 & 86.3 & 10 & 83.3 & 50 & 82.0 \\
\hline Black & 36 & 4.5 & 0 & 0 & 5 & 8.2 \\
\hline Asian & 15 & 1.9 & 1 & 8.3 & 3 & 4.9 \\
\hline Other/Biracial & 51 & 6.3 & 0 & 0 & 3 & 1.6 \\
\hline Missing & 8 & .10 & 1 & 8.3 & 0 & 0 \\
\hline $\begin{array}{l}\text { Ethnicity } \\
\text { Hispanic or }\end{array}$ & 32 & 4.0 & 0 & 0 & 1 & 1.6 \\
\hline Latino & & & & & & \\
\hline Not Hispanic & 739 & 91.9 & 100 & 100 & 59 & 96.7 \\
\hline or Latino & & & & & & \\
\hline Missing & 33 & 4.1 & 0 & 0 & 1 & 1.6 \\
\hline
\end{tabular}

*Note. One participant was not able to be weighed using the scale and therefore, this participant was excluded from the BMI calculations. 
Table 2

Additional Demographic characteristics of SONA (screening) sample

\begin{tabular}{|c|c|c|c|c|}
\hline & $\begin{array}{r}\text { SONA } \\
\text { Sam }\end{array}$ & ening & $\mathrm{Lal}$ & \\
\hline & $N$ & $\%$ & $n$ & $\%$ \\
\hline Family Income & & & & \\
\hline Less than $\$ 24,999$ & 53 & 6.6 & 5 & 8.2 \\
\hline$\$ 25,000$ to $\$ 34,9999$ & 48 & 6.0 & 7 & 11.5 \\
\hline$\$ 35,000$ to $\$ 49,999$ & 81 & 10.1 & 11 & 18.0 \\
\hline$\$ 50,000$ to $\$ 74,999$ & 146 & 18.2 & 13 & 21.3 \\
\hline$\$ 75,000$ to $\$ 99,000$ & 135 & 16.8 & 6 & 9.8 \\
\hline$\$ 100,000$ to $\$ 149,999$ & 173 & 21.5 & 10 & 16.4 \\
\hline Greater than $\$ 150,000$ & 123 & 15.3 & 7 & 11.5 \\
\hline Missing & 45 & 5.6 & 2 & 3.3 \\
\hline Father's Hx of Cardiovas & & & & \\
\hline Positive Hx & 76 & 9.5 & 6 & 9.8 \\
\hline Negative Hx & 696 & 86.6 & 54 & 88.5 \\
\hline Missing & 32 & 4.0 & 1 & 1.6 \\
\hline Father's Hx of Hypertens & & & & \\
\hline Positive Hx & 287 & 35.7 & 20 & 32.8 \\
\hline Negative Hx & 478 & 59.5 & 40 & 65.5 \\
\hline Missing & 39 & 4.9 & 1 & 1.6 \\
\hline Mother's Hx of Cardiova & & & & \\
\hline Disease & & & & \\
\hline Positive Hx & 40 & 5.0 & 52 & 85.2 \\
\hline Negative $\mathrm{Hx}$ & 746 & 92.8 & 8 & 13.1 \\
\hline Missing & 18 & 2.2 & 1 & 1.6 \\
\hline
\end{tabular}


Mother's Hx of Hypertension

Positive Hx

$188 \quad 23.4$

16

26.2

Negative Hx

601

74.8

44

72.1

Missing

15

1.9

1

1.6

Education

High School

$212 \quad 26.4$

17

1 year college

$240 \quad 29.9$

23

2 years college

$185 \quad 23.0$

7

11.5

3 years college

126

15.7

13

21.3

4 or more years college

37

4.6

1

1.6

Missing

4

0.5

0

0

*Note. Table includes demographic characteristics and means and standard deviations for variables collected thru SONA. Some participants declined to answer some questions. 
Table 3

Means and Standard Deviations for Study Variables

$\begin{array}{llll}\text { Mean } & S D & \text { Mean } & S D \\ \text { (untransformed) } & (\text { untransformed }) & \text { (transformed }) & \text { (transformed) }\end{array}$

Sample Characteristics

\begin{tabular}{|c|c|c|c|c|}
\hline Age (years) & 19.4 & 1.4 & 1.3 & .03 \\
\hline BMI & 25.48 & 5.31 & 1.40 & .08 \\
\hline Math Affinity* & 42.48 & 12.26 & - & - \\
\hline \multicolumn{5}{|c|}{ Cardiovascular Parameters during Baseline } \\
\hline SBP (mm Hg) & 112.5 & 8.68 & - & - \\
\hline $\mathrm{DBP}(\mathrm{mm} \mathrm{Hg})$ & 65.0 & 7.61 & - & - \\
\hline HR (bpm) & 79.8 & 12.83 & - & - \\
\hline LF-HRV (Hz) & 1942.99 & 3533.77 & 7.01 & .94 \\
\hline HF-HRV (Hz) & 1089.72 & 1014.74 & 6.60 & .93 \\
\hline \multicolumn{5}{|c|}{ Cardiovascular Parameters during Stress } \\
\hline $\mathrm{SBP}(\mathrm{mm} \mathrm{Hg})$ & 119.5 & 11.17 & - & - \\
\hline $\mathrm{DBP}(\mathrm{mm} \mathrm{Hg})$ & 71.6 & 8.07 & - & - \\
\hline HR (bpm) & 80.3 & 12.42 & - & - \\
\hline LF-HRV (Hz) & 1582.86 & 1742.92 & 7.01 & .80 \\
\hline HF-HRV (Hz) & 871.06 & 602.39 & 6.48 & .87 \\
\hline \multicolumn{5}{|c|}{ Cardiovascular Parameters during Recovery from Stress } \\
\hline $\mathrm{SBP}(\mathrm{mm} \mathrm{Hg})$ & 112.0 & 9.47 & - & - \\
\hline DBP (mm Hg) & 67.8 & 7.49 & - & - \\
\hline $\mathrm{HR}(\mathrm{bpm})$ & 77.1 & 11.74 & - & - \\
\hline LF-HRV (Hz) & 3720.10 & 3258.12 & 7.17 & .88 \\
\hline
\end{tabular}


HF-HRV (Hz)

2476.11

2162.68

6.60

1.50

*Note. Age and BMI are log transformed. LF and HRV HRV are natural-log transformed. 3 participants did not complete the Math Affinity questionnaire. 
Table 4

Univariate Correlations between Demographic, Cardiovascular, and Self-Reported Affect Variables

\begin{tabular}{|c|c|c|c|c|c|c|c|c|c|c|}
\hline & Age & $\operatorname{Sex}^{\mathrm{a}}$ & BMI & Math & NA B & PA B & NA RT & PA RT & NA RC & PA RC \\
\hline & & & & Affinity & & & & & & \\
\hline SBP-Rest $^{\mathrm{a}}$ & .01 & $-.30 *$ & .14 & -.01 & .14 & $.32 *$ & & & & \\
\hline DBP-Rest & .05 & -.01 & $.35 * *$ & -.14 & .01 & .17 & & & & \\
\hline HR-Rest & -.20 & .20 & .22 & -.02 & .00 & -.06 & & & & \\
\hline LF HRV-Rest & .03 & -.10 & -.05 & $.26^{*}$ & .12 & .24 & & & & \\
\hline HF HRV-Rest & .05 & .02 & -.04 & .09 & $.26^{*}$ & .09 & & & & \\
\hline SBP-Task $^{\mathrm{a}}$ & -.06 & $-.26 *$ & .14 & .07 & & & .23 & $.29 *$ & & \\
\hline DBP-Task & .13 & .07 & .001 & .04 & & & .09 & .04 & & \\
\hline HR-Task & -.22 & .19 & .13 & .02 & & & .09 & .04 & & \\
\hline LF HRV-Task & .02 & -.05 & -.04 & -.10 & & & .02 & -.06 & & \\
\hline HF HRV-Task & .05 & .10 & .04 & -.09 & & & .06 & -.17 & & \\
\hline $\mathrm{SBP}-\mathrm{RC}^{\mathrm{a}}$ & .04 & $-.30 *$ & .02 & -.06 & & & & & .21 & $.28 *$ \\
\hline $\mathrm{DBP}-\mathrm{RC}$ & .02 & -.07 & .06 & -.22 & & & & & .04 & .18 \\
\hline
\end{tabular}




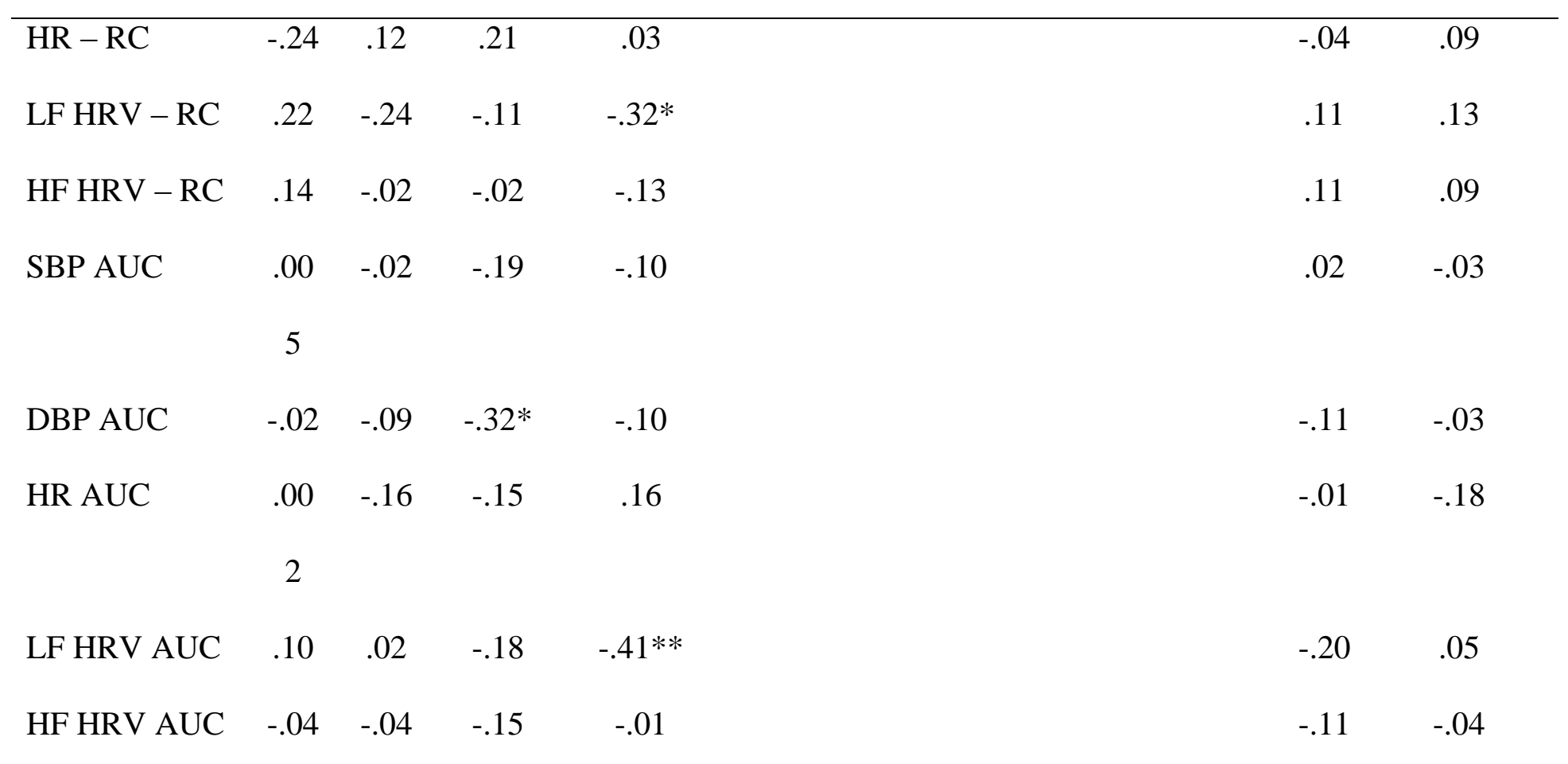

*Note. Age and BMI are log (base 10 transformed). HRV are natural-log transformed. NA is log (base 10) transformed. All other variables are untransformed. RC denotes recovery variables. ${ }^{\mathrm{a}}$ Men were found to have higher SBPs $(M=117.3 \mathrm{~mm} \mathrm{Hg}, S D=10.37)$ than women $(M=111.1 \mathrm{~mm} \mathrm{Hg}, S D=7.68) .(* p<.05 ; * * \mathrm{p} \leq .01)$. 
Table 5

Preliminary Analyses of SBP, DBP, and HR during each minute of the Resting Period

\begin{tabular}{|c|c|c|c|c|}
\hline & Mean & $S D$ & $F$ & $p$ \\
\hline SBP Minute 0 & 113.7 & 9.09 & & \\
\hline SBP Minute 2 & 112.3 & 8.47 & & \\
\hline SBP Minute 4 & 111.3 & 10.13 & & \\
\hline SBP Minute 6 & 112.2 & 9.52 & & \\
\hline SBP Minute 8 & 112.4 & 9.62 & & \\
\hline \multirow[t]{2}{*}{ SBP Minute 10} & 113.0 & 11.52 & & \\
\hline & & & 1.52 & .20 \\
\hline DBP Minute 0 & 65.1 & 8.24 & & \\
\hline DBP Minute 2 & 65.2 & 9.44 & & \\
\hline DBP Minute $4^{\mathrm{a}}$ & 63.7 & 9.77 & & \\
\hline DBP Minute 6 & 66.0 & 9.91 & & \\
\hline DBP Minute 8 & 65.2 & 9.64 & & \\
\hline \multirow[t]{2}{*}{ DBP Minute $10^{a}$} & 67.0 & 8.59 & & \\
\hline & & & 2.08 & .08 \\
\hline HR Minute $1^{\mathrm{b}, \mathrm{c}, \mathrm{d}}$ & 78.2 & 12.61 & & \\
\hline HR Minute $2^{\mathrm{b}, \mathrm{e}}$ & 80.8 & 13.36 & & \\
\hline HR Minute $3^{\mathrm{e}}$ & 78.9 & 13.17 & & \\
\hline HR Minute $4^{c}$ & 80.7 & 13.45 & & \\
\hline HR Minute 5 & 79.2 & 13.56 & & \\
\hline HR Minute 6 & 80.0 & 13.50 & & \\
\hline
\end{tabular}




$\begin{array}{lcc}\text { HR Minute } 7 & 78.8 & 12.71 \\ \text { HR Minute 8 } & 80.1 & 14.36 \\ \text { HR Minute 9 } & 79.8 & 14.86 \\ \text { HR Minute } 10^{\mathrm{d}} & 81.4 & 14.80\end{array}$

*Note. One-way repeated measures ANOVA for SBP, DBP, and HR (untransformed) across recordings made during the resting period. SBP and DBP are in $\mathrm{mm} \mathrm{Hg.} \mathrm{HR}$ is in bpm. Means with the same superscript are significantly different $(p<.05)$ 
Table 6

Preliminary Analyses of SBP, DBP, and HR during each minute of the StressorPeriod

\begin{tabular}{|c|c|c|c|c|}
\hline & Mean & $S D$ & $F$ & $p$ \\
\hline SBP Minute $0^{\mathrm{a}}$ & 120.8 & 12.65 & & \\
\hline SBP Minute 2 & 119.3 & 10.86 & & \\
\hline \multirow[t]{2}{*}{ SBP Minute $4^{\mathrm{a}}$} & 118.5 & 11.41 & & \\
\hline & & & 4.62 & .02 \\
\hline DBP Minute $0^{b}$ & 69.6 & 10.36 & & \\
\hline DBP Minute 2 & 72.0 & 10.17 & & \\
\hline \multirow[t]{2}{*}{ DBP Minute $4^{\mathrm{b}}$} & 73.1 & 8.36 & & \\
\hline & & & 4.35 & .02 \\
\hline HR Minute $1^{c}$ & 79.3 & 12.91 & & \\
\hline HR Minute $2^{c}$ & 81.0 & 12.70 & & \\
\hline HR Minute 3 & 80.0 & 12.69 & & \\
\hline HR Minute 4 & 80.4 & 13.30 & & \\
\hline \multirow[t]{2}{*}{ HR Minute 5} & 80.8 & 12.38 & & \\
\hline & & & 2.38 & .08 \\
\hline
\end{tabular}

*Note. One-way repeated measures ANOVA for SBP, DBP, and HR (untransformed) across recordings made during the stressor period. SBP and DBP are in $\mathrm{mm} \mathrm{Hg} \mathrm{HR}$ is in bpm. Means with the same superscript are significantly different $(p<.05)$. 
Table 7

Smile by Event ANOVAs on Math Performance and Affinity

\begin{tabular}{|c|c|c|c|c|c|}
\hline & Means & $S D$ & $F$ & $d f$ & $p$ \\
\hline \multicolumn{6}{|c|}{ Math Problems Presented } \\
\hline Smile & & & 1.03 & 1 & .32 \\
\hline Event & & & .08 & 1 & .77 \\
\hline Smile*Event & & & 1.42 & 1 & .24 \\
\hline Smile-Happy & 8.50 & .65 & & & \\
\hline Smile-Neutral & 7.58 & 2.43 & & & \\
\hline No-smile-Happy & 7.13 & 2.92 & & & \\
\hline Neutral & 7.69 & 2.36 & & & \\
\hline Error & & & & 50 & \\
\hline \multicolumn{6}{|c|}{ Problems Answered Correctly } \\
\hline Smile & & & 1.99 & 1 & .17 \\
\hline Event & & & .25 & 1 & .62 \\
\hline Smile*Event & & & 3.78 & 1 & .06 \\
\hline Smile-Happy & 2.79 & 2.42 & & & \\
\hline Smile-Neutral & 1.58 & 1.68 & & & \\
\hline No-smile-Happy & 1.13 & 1.41 & & & \\
\hline Neutral & 1.85 & 1.52 & & & \\
\hline Error & & & & 54 & \\
\hline
\end{tabular}




\begin{tabular}{|c|c|c|c|c|c|}
\hline & Means & $S D$ & $F$ & $d f$ & $p$ \\
\hline \multicolumn{6}{|l|}{$\%$ Answered Correctly } \\
\hline Smile & & & .32 & 1 & .58 \\
\hline Event & & & .16 & 1 & .70 \\
\hline Smile*Event & & & .008 & 1 & .93 \\
\hline Smile-Happy & 24.47 & 24.82 & & & \\
\hline Smile-Neutral & 21.71 & 15.64 & & & \\
\hline No-smile-Happy & 20.75 & 20.67 & & & \\
\hline Neutral & 19.01 & 20.22 & & & \\
\hline Error & & & & 50 & \\
\hline \multicolumn{6}{|l|}{ Math Affinity } \\
\hline Smile & & & .19 & 1 & .67 \\
\hline Event & & & .27 & 1 & .60 \\
\hline Smile*Event & & & 2.17 & 1 & .15 \\
\hline Smile-Happy & 41.79 & 12.50 & & & \\
\hline Smile-Neutral & 44.86 & 13.87 & & & \\
\hline No-smile-Happy & 45.14 & 11.37 & & & \\
\hline Neutral & 38.69 & 11.37 & & & \\
\hline Error & & & & 54 & \\
\hline
\end{tabular}

*Note. Univariate ANOVAs examining participants' performance on math problems and participants' scores on the Mental Arithmetic Questionnaire. Math problems presented refers to the number of math problems that participants were presented during the stressor task. Problems answered correctly refers to the total number of math problems that participants answered correctly. \% answered correctly is the percentage of math problems that participants answered 
correctly $(\%$ answered correctly $=$ math problems presented/problems answered correctly). Math Affinity is calculated by totaling the score on the Mental Arithmetic Questionnaire, which uses a scale of $1=$ Strongly disagree to $6=$ Strongly agree $(p<.05)$. 
Table 8

Reactivity to the Math Task for each dependent variable

\begin{tabular}{|c|c|c|c|c|c|}
\hline & Mean & $S D$ & $T$ & $d f$ & $p$ \\
\hline SBP Reactivity & & & -7.83 & 59 & $\leq .001$ \\
\hline $\mathrm{SBP}$ - rest & 112.8 & 8.49 & & & \\
\hline $\mathrm{SBP}$ - stressor & 119.5 & 11.17 & & & \\
\hline DBP Reactivity & & & -6.83 & 59 & $\leq .001$ \\
\hline $\mathrm{DBP}-$ rest & 65.1 & 7.66 & & & \\
\hline DBP - stressor & 71.6 & 8.07 & & & \\
\hline HR Reactivity & & & -.94 & 56 & .35 \\
\hline $\mathrm{HR}$ - rest & 79.5 & 12.75 & & & \\
\hline HR - stressor & 80.3 & 12.42 & & & \\
\hline LF HRV Reactivity & & & .02 & 57 & .99 \\
\hline LF HRV - rest & 7.04 & .94 & & & \\
\hline LF HRV - stressor & 7.03 & .80 & & & \\
\hline HF HRV Reactivity & & & .81 & 57 & .42 \\
\hline HF HRV - rest & 6.63 & .94 & & & \\
\hline HF HRV - stressor & 6.51 & .82 & & & \\
\hline NA Reactivity & & & -6.07 & 60 & $\leq .001$ \\
\hline $\mathrm{NA}$ - rest & 1.07 & .07 & & & \\
\hline NA - stressor & 1.15 & .13 & & & \\
\hline
\end{tabular}




\begin{tabular}{|c|c|c|c|c|c|}
\hline & Mean & $S D$ & $T$ & $d f$ & $p$ \\
\hline PA Reactivity & & & 2.72 & 59 & .009 \\
\hline $\mathrm{PA}$ - rest & 23.33 & 6.47 & & & \\
\hline PA - stressor & 21.35 & 5.68 & & & \\
\hline
\end{tabular}

*Note. Paired samples t-tests were conducted to examine reactivity. SBP and DBP are in mm Hg.

$\mathrm{HR}$ is in bpm. HRV is in $\mathrm{Hz}$ and is natural-log transformed. PA and NA are calculated by totaling scores from items measuring general PA and NA on the PANAS-X. Items on the PANAS-X are measured on a Likert-type scale $(1=$ Very slightly or not at all to $5=$ Extremely $)$. NA is $\log$ (base 10) transformed. All other data is untransformed $(p<.05)$. 
Table 9

Means, Standard Deviations, and ANOVA Summary Tables for SBP and DBP during the Resting Period

\begin{tabular}{|c|c|c|c|c|c|}
\hline & Means & $S D$ & $F$ & $d f$ & $p$ \\
\hline \multicolumn{6}{|l|}{ SBP - baseline } \\
\hline Smile & & & .36 & 1 & .55 \\
\hline Event & & & .006 & 1 & .94 \\
\hline Smile*Photo & & & .001 & 1 & .98 \\
\hline Smile-Happy & 113.1 & 9.08 & & & \\
\hline Smile-Neutral & 113.3 & 8.77 & & & \\
\hline No-Smile-Happy & 111.7 & 9.24 & & & \\
\hline Neutral & 111.9 & 8.49 & & & \\
\hline Error & & & & 57 & \\
\hline \multicolumn{6}{|l|}{ DBP - baseline } \\
\hline Smile & & & .16 & 1 & .69 \\
\hline Event & & & .08 & 1 & .77 \\
\hline Smile*Photo & & & 5.37 & 1 & .02 \\
\hline Smile-Happy & 63.0 & 4.96 & & & \\
\hline Smile-Neutral & 68.0 & 7.65 & & & \\
\hline No-Smile-Happy & 66.6 & 7.63 & & & \\
\hline Neutral & 62.8 & 8.69 & & & \\
\hline Error & & & & 57 & \\
\hline
\end{tabular}

*Note. SBP and DBP are in $\mathrm{mm} \mathrm{Hg}(p<.05)$. 
Table 10

Simple Main Effects of DBP for the Smile by Event Interaction during the Resting Period

\begin{tabular}{llcccc}
\hline & Means & $S D$ & $f$ & $d f$ & $p$ \\
\hline Happy Photographs & & & 1.76 & 1 & .19 \\
Smile Happy & 63.0 & 4.96 & & \\
No-Smile-Happy & 66.6 & 7.63 & & \\
Error & & & 57 & \\
Neutral Photographs & & & 3.87 & 1 & .05 \\
Smile-Neutral & 68.0 & 7.65 & & \\
Neutral & 62.8 & 8.70 & & \\
Error & & & & \\
\end{tabular}

*Note. DBP is in $\mathrm{mm} \mathrm{Hg}(p<.05)$. 
Table 11

Means, Standard Deviations, and ANOVA Summary Tables for SBP and DBP during the Stressor Period

\begin{tabular}{|c|c|c|c|c|c|}
\hline & Means & $S D$ & $F$ & $d f$ & $p$ \\
\hline \multicolumn{6}{|l|}{ SBP - reactivity } \\
\hline Smile & & & .13 & 1 & .72 \\
\hline Event & & & 4.30 & 1 & .04 \\
\hline Smile*Photo & & & .77 & 1 & .39 \\
\hline Smile-Happy & 124.3 & 12.01 & & & \\
\hline Smile-Neutral & 118.0 & 9.33 & & & \\
\hline No-Smile-Happy & 119.3 & 12.45 & & & \\
\hline Neutral & 117.5 & 10.72 & & & \\
\hline Error & & & & 55 & \\
\hline \multicolumn{6}{|l|}{$\mathrm{DBP}$ - reactivity } \\
\hline Smile & & & 7.56 & 1 & .008 \\
\hline Event & & & .28 & 1 & .602 \\
\hline Smile*Photo & & & .09 & 1 & .77 \\
\hline Smile-Happy & 72.6 & 7.28 & & & \\
\hline Smile-Neutral & 75.8 & 7.40 & & & \\
\hline No-Smile-Happy & 69.6 & 9.02 & & & \\
\hline Neutral & 68.7 & 7.13 & & & \\
\hline Error & & & & 55 & \\
\hline
\end{tabular}

*Note. Pre-Task resting cardiovascular parameters were included as covariates. SBP and DBP are in $\mathrm{mm} \mathrm{Hg}(p<.05)$. 
Table 12

Means, Standard Deviations, and ANOVA Summary Tables for SBP and DBP Areas Under the Curve during the Recovery Period of Blood Pressure Controlling for Law of Initial Values and Reactivity

$\begin{array}{lllll}\text { Means } & S D & F & d f\end{array}$

\begin{tabular}{|c|c|c|c|c|c|}
\hline \multicolumn{6}{|l|}{$\mathrm{SBP}-\mathrm{AUC}$} \\
\hline Smile & & & .34 & 1 & .57 \\
\hline Event & & & 3.80 & 1 & .06 \\
\hline Smile*Photo & & & .27 & 1 & .61 \\
\hline Smile-Happy & -35.4 & 2246.70 & & & \\
\hline Smile-Neutral & -1225.3 & 3442.48 & & & \\
\hline No-Smile-Happy & 1013.3 & 3524.08 & & & \\
\hline Neutral & -1087.1 & 2992.27 & & & \\
\hline Error & & & & 54 & \\
\hline \multicolumn{6}{|l|}{$\mathrm{DBP}-\mathrm{AUC}$} \\
\hline Smile & & & .06 & 1 & .82 \\
\hline Event & & & 1.15 & 1 & .29 \\
\hline Smile*Photo & & & .49 & 1 & .49 \\
\hline Smile-Happy & 3665.00 & 4523.64 & & & \\
\hline Smile-Neutral & 876.00 & 3968.03 & & & \\
\hline No-Smile-Happy & 900.00 & 2919.55 & & & \\
\hline Neutral & 1630.59 & 4247.69 & & & \\
\hline Error & & & & 52 & \\
\hline
\end{tabular}


Table 13

Means, Standard Deviations, and ANOVA Summary Tables for Time to Recovery to Initial Resting SBP and DBP

Means $\quad S D \quad F \quad d f \quad p$

SBP

Smile

1.99

1

.16

Event

.91

1

.34

Smile*Photo

.02

1

.88

$\begin{array}{lll}\text { Smile-Happy } & 4.00 & 3.66 \\ \text { Smile-Neutral } & 3.00 & 2.83 \\ \text { No-Smile-Happy } & 5.13 & 4.10 \\ \text { Neutral } & 4.41 & 3.37\end{array}$

Error

57

DBP

Smile

.60

.44

Event

.00

.95

Smile*Photo

1.86

.18

$\begin{array}{lcc}\text { Smile-Happy } & 6.86 & 4.61 \\ \text { Smile-Neutral } & 5.40 & 4.36 \\ \text { No-Smile-Happy } & 4.47 & 4.31 \\ \text { Neutral } & 6.06 & 4.19\end{array}$

Error

57

*Note. Smile by Event ANOVAs examining the main effects of time (in min) to return to resting levels of SBP and DBP. 
Table 14

Means, Standard Deviations, and ANOVA Summary Tables for NA and PA following Resting, Stress, and Recovery Periods

\begin{tabular}{|c|c|c|c|c|c|c|c|}
\hline & Means $^{a}$ & $S D^{a}$ & Means $^{b}$ & $S D^{b}$ & $F$ & $d f$ & $p$ \\
\hline \multicolumn{8}{|l|}{$\mathrm{NA}$ - baseline } \\
\hline Smile & & & & & .77 & 1 & .38 \\
\hline Event & & & & & .09 & 1 & .77 \\
\hline Smile*Photo & & & & & .06 & 1 & .81 \\
\hline Smile-Happy & 11.71 & 2.30 & 1.06 & .08 & & & \\
\hline Smile-Neutral & 11.67 & 1.68 & 1.06 & .06 & & & \\
\hline No-Smile-Happy & 12.07 & 2.43 & 1.07 & .08 & & & \\
\hline Neutral & 12.35 & 2.40 & 1.09 & .08 & & & \\
\hline Error & & & & & & 57 & \\
\hline \multicolumn{8}{|l|}{ NA - stressor } \\
\hline Smile & & & & & .34 & 1 & .56 \\
\hline Event & & & & & .02 & 1 & .88 \\
\hline Smile*Photo & & & & & .11 & 1 & \\
\hline Smile-Happy & 15.57 & 5.71 & 1.17 & .15 & & & \\
\hline Smile-Neutral & 15.00 & 4.04 & 1.16 & .12 & & & \\
\hline No-Smile-Happy & 14.33 & 4.94 & 1.14 & .13 & & & \\
\hline Neutral & 15.06 & 6.09 & 1.15 & .14 & & & \\
\hline
\end{tabular}

Error 


$\begin{array}{llllll}\text { Means }^{a} & S D^{a} & \text { Means }^{b} & S D^{b} & F & d f\end{array}$

$\mathrm{NA}-$ recovery

Smile

Event

Smile*Photo

Smile-Happy

11.64

11.68

Smile-Neutral

No-Smile-Happy

Neutral

11.60

11.94

Error

PA - baseline

Smile

Event

Smile*Photo

Smile-Happy

23.36

Smile-Neutral

21.73

23.07

24.24

Neutral

Error
$.01 \quad 1 \quad .95$

$\begin{array}{lll}.16 & 1 & .69\end{array}$

$\begin{array}{lll}.11 & 1 & .75\end{array}$

.06

.06

.08

.08

$\begin{array}{lll}.41 & 1 & .52 \\ .02 & 1 & .90 \\ .66 & 1 & .42\end{array}$

7.56

6.13

7.19

5.98 


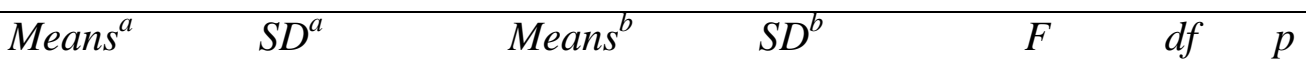

PA - stressor

Smile

$\begin{array}{lll}1.89 & 1 & .17\end{array}$

Event

$\begin{array}{lll}.13 & 1 & .72\end{array}$

Smile*Photo

$.31 \quad 1 \quad .58$

Smile-Happy

20.14

7.35

Smile-Neutral

20.43

5.65

No-Smile-Happy

23.00

4.18

Neutral

21.65

5.42

Error

56

PA - recovery

Smile

$\begin{array}{lll}1.75 & 1 & .19\end{array}$

Event

$\begin{array}{lll}.17 \quad & 1\end{array}$

Smile*Photo

$\begin{array}{lll}.04 & 1 & .85\end{array}$

Smile-Happy

17.71

6.08

Smile-Neutral

18.73

6.46

No-Smile-Happy

20.27

6.76

Neutral

20.65

6.91

Error

57

*Note. Means ${ }^{a}$ and $\mathrm{SD}^{a}$ are based on untransformed data and Means ${ }^{b}$ and $\mathrm{SD}^{b}$ are based on transformed data. NA is log (base 10) transformed. PA is untransformed $(p<.05)$. 
Table 15

Means, Standard Deviations, and ANOA Summary Tables on Items from the Post-Experiment Questionnaire

\begin{tabular}{|c|c|c|c|c|c|}
\hline & Means & $S D$ & $F$ & $d f$ & $p$ \\
\hline \multicolumn{6}{|l|}{ Difficulty } \\
\hline Smile & & & 1.41 & 1 & .24 \\
\hline Event & & & .46 & 1 & .50 \\
\hline Smile*Photo & & & .23 & 1 & .63 \\
\hline Smile-Happy & 45.36 & 32.79 & & & \\
\hline Smile-Neutral & 43.93 & 26.11 & & & \\
\hline No-Smile-Happy & 57.67 & 30.87 & & & \\
\hline Neutral & 49.12 & 23.86 & & & \\
\hline Error & & & & 56 & \\
\hline \multicolumn{6}{|l|}{ Tiredness } \\
\hline Smile & & & 1.53 & 1 & .22 \\
\hline Event & & & .002 & 1 & .97 \\
\hline Smile*Photo & & & .12 & 1 & .73 \\
\hline Smile-Happy & 59.64 & 27.42 & & & \\
\hline Smile-Neutral & 61.79 & 24.85 & & & \\
\hline No-Smile-Happy & 53.33 & 31.32 & & & \\
\hline Neutral & 50.59 & 25.30 & & & \\
\hline
\end{tabular}




\section{Means \\ $S D$ \\ F \\ $d f$ \\ $p$}

\section{Comfort}

Smile

Event

Smile*Photo

Smile-Happy

58.21

Smile-Neutral

59.67

63.33

52.06

Error

Distraction

Smile

Event

Smile*Photo

Smile-Happy

39.64

29.29

38.67

24.41

Neutral

Error

19.26

26.81

22.64

21.50
26.76

$\begin{array}{lll}.04 & 1 & .85\end{array}$

$\begin{array}{lll}.54 & 1 & .47\end{array}$

$\begin{array}{lll}.91 & 1 & .35\end{array}$

27.15

26.62

23.50

57

$\begin{array}{lll}.25 & 1 & .62\end{array}$

$\begin{array}{lll}4.40 & 1 & .04\end{array}$

$\begin{array}{lll}.11 & 1 & .74\end{array}$

56

*Note. Difficulty, Tiredness, Comfort, and Distraction are measured on scales of $0 \%=$ Not at all to $100 \%=$ Very difficult/Very Tired/Very Uncomfortable/Very distracted $(p<.05)$. 
Table 16

Means, Standard Deviations, and ANOVA Summary Tables on Items Assessing Nature of Distracting Thoughts during the Task from the Post-Experiment Questionnaire

\begin{tabular}{|c|c|c|c|c|c|}
\hline & Means & $S D$ & $F$ & $d f$ & $p$ \\
\hline Item 1 - Impatience & & & .14 & 1 & .71 \\
\hline Smile & & & 1.16 & 1 & .29 \\
\hline Event & & & 2.86 & 1 & .10 \\
\hline \multicolumn{6}{|l|}{ Smile*Photo } \\
\hline Smile-Happy & 2.36 & 1.01 & & & \\
\hline Smile-Neutral & 2.53 & 1.30 & & & \\
\hline No-Smile-Happy & 2.73 & 1.28 & & & \\
\hline Neutral & 1.94 & .83 & & & \\
\hline Error & & & & 57 & \\
\hline \multicolumn{6}{|l|}{ Item $2-$ Absorption } \\
\hline Smile & & & 2.32 & 1 & .13 \\
\hline Event & & & .19 & 1 & .66 \\
\hline Smile*Photo & & & .13 & 1 & .72 \\
\hline Smile-Happy & 4.57 & 1.70 & & & \\
\hline Smile-Neutral & 4.87 & 1.77 & & & \\
\hline No-Smile-Happy & 5.27 & 1.03 & & & \\
\hline Neutral & 5.29 & 1.16 & & & \\
\hline Error & & & & 57 & \\
\hline
\end{tabular}




\section{$\begin{array}{lllll}\text { Means } & S D & F & d f\end{array}$}

Item 3 - Relaxing

Smile

Event

Smile*Photo

Smile-Happy

2.36

Smile-Neutral

2.07

2.33

1.45

Neutral

2.06

.97

Error

Item 4 - Pleasant Thoughts

Smile

Event

Smile*Photo

Smile-Happy

2.93

1.49

Smile-Neutral

2.14

1.29

No-Smile-Happy

2.93

1.62

Neutral

2.29

.99

1.55

1.10

\section{5}

Error

$\begin{array}{lll}.002 & 1 & .96 \\ .75 & 1 & .39 \\ .001 & 1 & .98\end{array}$


$\begin{array}{lllll}\text { Means } & \text { SD } & F & d f\end{array}$

Item 5 - Enjoyment

Smile

$\begin{array}{ll}.05 & 1\end{array}$

.83

Event

1.30

1

.26

Smile*Photo

.45

1

.50

$\begin{array}{lll}\text { Smile-Happy } & 2.07 & 1.07 \\ \text { Smile-Neutral } & 2.21 & 1.42 \\ \text { No-Smile-Happy } & 1.80 & .78 \\ \text { Neutral } & 2.35 & 1.32\end{array}$

Error

56

Item 6 - Planning Next

Smile

.91

.001

1

.34

Event

Smile*Photo

4.05

1.04

Smile-Happy

2.00

1.62

No-Smile-Happy

3.07

1.44

Neutral

2.35

1.41

Error 


\begin{tabular}{|c|c|c|c|c|c|}
\hline & Means & $S D$ & $F$ & $d f$ & $p$ \\
\hline \multicolumn{6}{|c|}{ Item 7 - Waste of Time } \\
\hline Smile & & & 1.09 & 1 & .30 \\
\hline Event & & & .25 & 1 & .62 \\
\hline Smile*Photo & & & .96 & 1 & .33 \\
\hline Smile-Happy & 1.50 & .94 & & & \\
\hline Smile-Neutral & 1.87 & .83 & & & \\
\hline No-Smile-Happy & 2.00 & 1.20 & & & \\
\hline Neutral & 1.88 & .86 & & & \\
\hline Error & & & & 57 & \\
\hline \multicolumn{6}{|l|}{ Item 8 - Long Time } \\
\hline Smile & & & 1.52 & 1 & .22 \\
\hline Event & & & 3.42 & 1 & .07 \\
\hline Smile*Photo & & & 4.42 & 1 & .04 \\
\hline Smile-Happy & 2.43 & 1.34 & & & \\
\hline Smile-Neutral & 2.53 & 1.85 & & & \\
\hline No-Smile-Happy & 3.80 & 1.94 & & & \\
\hline Neutral & 2.18 & 1.19 & & & \\
\hline Error & & & & 57 & \\
\hline
\end{tabular}

*Note. Participants rated eight items on a Likert-type scale from $1=$ Strongly Disagree to $7=$ Strongly Agree $(p<.05)$. 
Table 17

Simple Main Effects for Smile by Event Interactions on Items 6 and 8 on the Post-Experiment Questionnaire

Means $\quad S D \quad F \quad d f \quad p$

Item 6 - Planning Next

Happy Photographs

$4.20 \quad 1 \quad .05$

Smile-Happy

$2.00 \quad 1.01$

No-Smile-Happy

3.07

1.44

Error

Neutral Photographs

$2.73 \quad 1.62$

Smile-Neutral

1.41

Neutral

\subsection{5}

.62

Error

57

Item 8 - Long Time

Happy Photographs

$5.32 \quad 1$

57

$.59 \quad 1$

.45

Smile-Happy

1.34

No-Smile-Happy

3.80

1.94

\section{Error}

Neutral Photographs

Smile-Neutral

2.53

2.18

Neutral

1.19

Error

*Note. Participants rated items on a Likert-type scale from $1=$ Strongly Disagree to $7=$ Strongly Agree $(p<.05)$. 
Table 18

Ratings of Positive and Neutral Photographs on the Post-Experimental Questionnaire

\begin{tabular}{lccccc}
\hline & Mean & $S D$ & $t$ & $d f$ & \\
\hline Positive Ratings $^{\mathrm{a}}$ & & & .49 & 27 & \\
Smile-Happy & 7.79 & 1.63 & & \\
No-smile-Happy & 7.40 & 2.50 & & \\
Neutral Ratings & & & & \\
Smile-Neutral & 7.93 & 1.33 & & & \\
Neutral & 8.35 & 1.06 & & \\
\end{tabular}

*Note. Independent samples t-test examining ratings of positive (personally-relevant) and neutral (non-personally relevant) photographs. ${ }^{\text {a}}$ Participants rated positive photographs on a scale from 1 $=$ Not at all happy to $9=$ Extremely happy. ${ }^{\mathrm{b}}$ Participants rated neutral photographs on a scale from $1=$ Not at all neutral to $9=$ Extremely neutral $(p<.05)$. 
Table 19

Group Differences in Knowledge of Smile Effect from the Post-Experiment Questionnaire

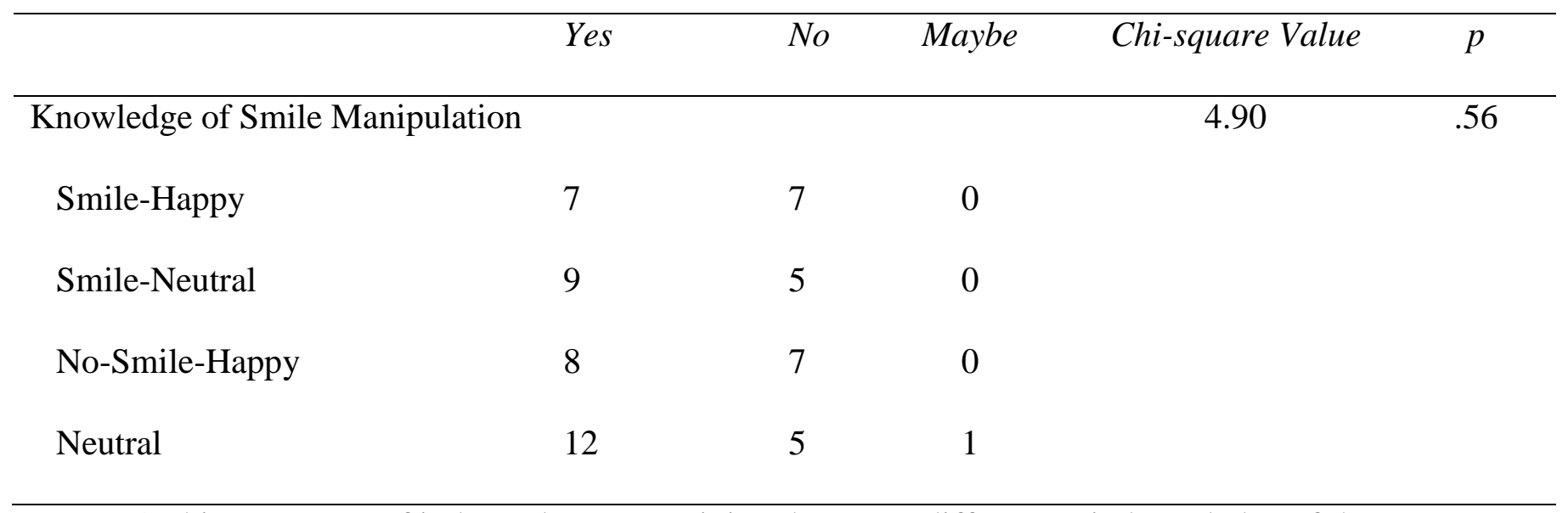

*Note. A chi-square test of independence examining the group differences in knowledge of the true reason for the Smile manipulation task $(p<.05)$. 


\section{Appendix A}

\section{Pilot Study}

A pilot study was conducted prior to the main study to examine the procedure and stimuli used in the main laboratory phase. The pilot study included 12 undergraduates (three men and nine women with a $M_{\text {age }}=19.3$ years, $S D=.78$ ) recruited from the SONA screening phase sample (see Table 1). The Smile-Happy Group included 5 participants; the Smile-Neutral Group included 3 participants; the No-smile-Happy Group included 2 participants; and the No-smileNeutral Group included 2 participants. The sample included 10 white participants, 1 Asian participant, and 1 participant that did not disclose his/her race. The sample did not include any participants that identified their ethnicity as Hispanic or Latino.

Based upon results of the pilot study, the procedure for the main laboratory phase was modified in two ways. In the pilot study, the PANAS-X, was given only two times, following the Resting and Stressor periods. For the main study, the PANAS-X was given three times, following the Resting, Stressor, and Recovery periods. It was determined that the PANAS-X should be given following each period in the main study to obtain additional data regarding changes in affect that occurred during the recovery period. Second, during the pilot study it was observed that participants failed to maintain the appropriate facial expression (Duchenne-smile vs. no-smile) throughout the entire stressor period. Therefore, a reminder prompting participants to continue to engage in this treatment was added to the main study. Additionally, during the pilot study it was observed that some participants discontinued efforts to answer the mental arithmetic questions. To ensure that the mental arithmetic stressor task produced increases in physiological responses, it was important that participants attempted to complete the mental arithmetic questions consistently; therefore, a reminder for participants to try their best was 
added for participants in the main study. No other modifications were made based upon findings from the pilot study. Based upon feedback from pilot study participants, instructions for positioning chopsticks for purposes of manipulating facial muscles and completing the study tasks were clear and functioned as intended. 


\section{Appendix B}

\section{Demographics Questionnaire}

Participant \#:

Height(in.):

\section{Your Information:}

Your age

Your sex

$$
\begin{aligned}
& \circ \text { Male } \\
& \circ \text { Female }
\end{aligned}
$$

Date:

Weight(lbs):

Your race

○ Black

$\circ$ White

○ Asian

$\circ$ Biracial

o Other

If you answered Biracial to the above question please specify:

If you answered Other to the above question please specify:

Your ethnicity

○ Not Hispanic or Latino

○ Hispanic or Latino

Indicate the highest level of education you have completed:

$\circ$ High school

○ 1 year college

○ 2 years college

○ 3 years college

○ 4 or more years college 
Please describe any cardiovascular related illness that you may have, including high blood pressure:

Please list any other medical or psychiatric problems that you have:

Please list any major surgeries and medical, or psychiatric illnesses you have had in the past.

Females: Are you currently pregnant?

$\circ$ Yes

$\circ$ No

Females: Are you currently on birth control (contraceptives).

$\circ$ No

$\circ$ Yes

What type of birth control are you taking?

Please list any drugs (legal or otherwise) that you are currently taking including; birth control (contraceptives), heart medications, cold or allergy medications, over the counter medications, asthma medications, Beta-Blockers (i.e. Inderal, Tenormin), psychoactive drugs (i.e. Adderall, Xanax, Haldol, Lithium, Prozac), or diet pills.

Do you currently smoke cigarettes (within the last month)?

$$
\begin{aligned}
& \circ \text { Yes } \\
& \circ \text { No }
\end{aligned}
$$

Do you currently use smokeless tobacco (within the past month)?

$$
\begin{aligned}
& \circ \text { Yes } \\
& \circ \text { No }
\end{aligned}
$$

How often do you drink alcohol?

$$
\begin{aligned}
& \circ \text { never } \\
& \circ \text { infrequently (a few drinks per year) }
\end{aligned}
$$




$$
\begin{aligned}
& \text { occasionally (1-2 drinks per month) } \\
& \text { o weekly (1-3 drinks per week) } \\
& \text { o weekly (3-6 drinks per week) } \\
& \text { o daily (7-14 drinks per week) }
\end{aligned}
$$

How many cups of caffeinated coffee, tea, or soda do you have per day?

$$
\begin{aligned}
& \circ \text { none } \\
& \circ \text { 1-2 cups per day } \\
& \circ \text { 3-4 cups per day } \\
& \circ 5-6 \text { cups per day } \\
& \circ \text { 7-8 cups per day } \\
& \circ \text { greater than eight cups per day }
\end{aligned}
$$

How many times per week do you engage in aerobic physical activity?

$$
\begin{aligned}
& \text { never } \\
& \text { 1-2 times } \\
& \text { 3-6 times } \\
& \text { } 7 \text { or more times }
\end{aligned}
$$

For how long do you typically exercise on each occasion?

$$
\begin{aligned}
& \text { 5-10 minutes } \\
& \text { 10-15 minutes } \\
& \text { 15-30 minutes } \\
& \text { 30-60 minutes } \\
& \text { more than } 60 \text { minutes }
\end{aligned}
$$

\section{Family Information:}

What is your best estimate of your family's total income? 
○ Less than 24,999

○ 25,000 to 34,999

○ 35,000 to 49,999

○ 50,000 to 74,999

○ 75,000 to 99,999

○ 100,000 to 149,999

○ Greater than 150,000

Is your father currently living?

○ yes

○ no

Approximately how old is your father?

Did/does your father have high blood pressure (hypertension)?

$\circ$ yes

o no

How certain are you that he did, or did not, have high blood pressure (hypertension)?

○ Absolutely (100\%) certain

$\circ$ Almost (75\%) certain

$\circ$ Not sure at all $(25 \%)$

○ No information by which to judge $(0 \%)$

Did/does your father have any heart problems such as angina (chest pains), a heart attack, or coronary heart disease?

$$
\begin{aligned}
& \circ \text { yes } \\
& \circ \text { no }
\end{aligned}
$$

If yes, please specify if you are able:

How certain are you that he did, or did not, have a heart problem as indicated above?

o Absolutely (100\%) certain 
○ Almost (75\%) certain

$\circ$ Not sure at all $(25 \%)$

$\circ$ No information by which to judge $(0 \%)$

Is your mother currently living?

$$
\begin{aligned}
& \circ \text { yes } \\
& \circ \text { no }
\end{aligned}
$$

Approximately how old is your mother?

Did/does your mother have high blood pressure (hypertension)?

$$
\begin{aligned}
& \circ \text { yes } \\
& \circ \text { no }
\end{aligned}
$$

How certain are you that she did, or did not, have high blood pressure (hypertension)?

$$
\begin{aligned}
& \circ \text { Absolutely }(100 \%) \text { certain } \\
& \circ \text { Almost }(75 \%) \text { certain } \\
& \circ \text { Not sure at all }(25 \%) \\
& \circ \text { No information by which to judge }(0 \%)
\end{aligned}
$$

Did/does your mother have any heart problems such as angina (chest pains), a heart attack, or coronary heart disease?

$$
\begin{aligned}
& \circ \text { yes } \\
& \circ \text { no }
\end{aligned}
$$

If yes, please specify if you are able:

How certain are you that she did, or did not, have a heart problem as indicated above?

$$
\begin{aligned}
& \circ \text { Absolutely (100\%) certain } \\
& \circ \text { Almost (75\%) certain } \\
& \circ \text { Not sure at all }(25 \%)
\end{aligned}
$$


○ No information by which to judge $(0 \%)$

Please provide your email address (you will contacted through this email address if you are eligible for Part 2 of the study): 


\section{Appendix C}

\section{Mental Arithmetic Questionnaire}

The Mental Arithmetic Questionnaire was adapted from Salvia et al. (2013). The language for several items and the anchor points for the Likert-type scale were changed. 


\section{Appendix D}

\section{Arithmetic Calculations}

Instructions for the arithmetic calculations were adapted from (Salvia et al., 2013).

- In a moment you will be presented with a series of calculations. Some are easy calculations and some are more difficult calculations. In each set of calculations you will first see a preparation screen with the $\mathrm{X}$ pictures (personally-relevant photographs or nonpersonally relevant photographs depending on the condition to which they are randomized) which will be presented for a brief period of time. Immediately following the preparation screen you will see the calculation screen. You will be given a period of time to complete the calculation, which will be visible to you on the screen. The time available to you may adjust for easy and difficult calculations. After you have calculated the answer please enter it into the textbox and click the "ENTER" button. You will then see a message box that tells you if your answer is right or wrong. If you answered correctly, the preparation screen will return, followed by a new math calculation. If you answered incorrectly, you will receive a message telling you that you are wrong. If time remains you may attempt to complete the question again. The goal is to work quickly, but to also ensure your accuracy.

- Any questions?

The calculations used in the present study were originally developed by Salvia et al. (2013). Easy and difficult math problems were used in the present study. 
Appendix E

Smile and No-Smile Manipulation Positioning and Instructions

See Kraft and Pressman (2012) for photographs used for training of Duchenne-smile and Nosmile positioning.

Instructions provided to participants randomized to the Duchenne-smile group.

- In a moment you will begin the calculation task. Before beginning the task, we would like to show you the other task that you will be performing. In this task, you will hold these chopsticks sideways in your mouth. The chopsticks should be positioned between your teeth. (See above picture as an example).

- Remember the purpose of the study is to examine the effects of multitasking on completing these calculations, so please maintain this position throughout the study.

Instructions provided to participants randomized to the Duchenne-smile group.

- In a moment you will begin the calculation task. Before beginning the task, we would like to show you the other task that you will be performing. In this task, you will hold these chopsticks in your mouth. The chopsticks should be positioned between your upper lip. (See above picture as an example).

- Remember the purpose of the study is to examine the effects of multitasking on completing these calculations, so please maintain this position throughout the study. 


\section{Appendix F}

\section{IAPS}

The images from the IAPS are copyright and cannot be duplicated (Lang et al., 1995). 


\section{Appendix G}

\section{Event Instructions}

Instructions provided to participants randomized to the personally-relevant photograph group regarding selecting a photograph to send to the experimenter.

- The purpose of this study is to examine the effects of multitasking on completing math calculations. If you are interested in participating in this study, we ask that you email us three photographs that reminds you of a pleasant memory. For example, you may select photographs of a birthday party, a vacation, etc.; however, the three photographs must be from the same event. Please choose an appropriate photograph that you are comfortable sharing. During this study you will be presented with these photographs as you complete a series of tasks.

Instructions provided to the participants randomized to the personally-relevant photograph group prior to beginning the study.

- In addition to the calculations that you will be performing, there will be photographs presented before each math problem. These are the photographs that you provided to us.

- Remember the purpose of the study is to examine the effects of multitasking on completing these calculations, so please concentrate on the photographs and recall the memory associated with the photographs while working on these math problems.

Instructions provided to participants randomized to the non-personally relevant photograph group prior to beginning the study.

- In addition to the calculations that you will be performing, there will be photographs presented before each math problem.

- Remember the purpose of the study is to examine the effects of multitasking on completing these calculations, so please concentrate on the photographs throughout the study. 


\section{Appendix $\mathrm{H}$}

\section{Description of the Study}

The following description was provided to participants regarding the purpose of the study. This description was adapted from two previous studies examining the facial feedback hypothesis (Kraft \& Pressman, 2012; Strack et al., 1988).

- "The purpose of this study is to examine the effects of multitasking on performance in completing math calculations. In a moment you will begin a series of math calculations. During the calculations you will be asked to engage in... (group that they are randomized to). While you are completing the math calculations we ask that you continue to engage in... (X task that they are randomized to)" 


\section{Appendix I}

\section{Debriefing}

In this study you engaged in...(group that they are randomized to). We told you that we were interested in the effects of multitasking on performance in completing math calculations. However, we are actually examining the effects of positive emotions on physiological responses during stressful tasks. The math calculations were designed to be stressful and the task .... (group they are randomized to) that you engaged in was designed to evoke (positive or neutral emotions depending on the condition they are randomized to) emotions. Recent research demonstrated that positive emotions may speed physiological recovery from stressors. Therefore, we were interested in the examining two methods of inducing positive emotions to determine if one method assists physiological recovery better than the other.

Any questions?

Thank you for your participation. 
Appendix J

Post-Experiment Questionnaire-A

Please indicate your agreement with the following statements about the math task (where you were completing math problems).

\begin{tabular}{|l|c|c|c|c|c|c|c|}
\hline & $\begin{array}{l}\text { Strongly } \\
\text { Disagree }\end{array}$ & & & & & & $\begin{array}{l}\text { Strongly } \\
\text { Agree }\end{array}$ \\
\hline $\begin{array}{l}\text { I was impatient for } \\
\text { the task to end so I } \\
\text { could finish the } \\
\text { study. }\end{array}$ & 1 & 2 & 3 & 4 & 5 & 6 & 7 \\
\hline $\begin{array}{l}\text { My thoughts were } \\
\text { completely } \\
\text { absorbed by the } \\
\text { task. }\end{array}$ & 1 & 2 & 3 & 4 & 5 & 6 & 7 \\
\hline $\begin{array}{l}\text { I felt the task was a } \\
\text { relaxing break. }\end{array}$ & 1 & 2 & 3 & 4 & 5 & 6 & 7 \\
\hline $\begin{array}{l}\text { The task inspired } \\
\text { me to think } \\
\text { pleasant thoughts. }\end{array}$ & 1 & 2 & 3 & 4 & 5 & 6 & 7 \\
\hline $\begin{array}{l}\text { I was enjoying the } \\
\text { task and I did not } \\
\text { want it to end. }\end{array}$ & 1 & 2 & 3 & 4 & 5 & 6 & 7 \\
\hline $\begin{array}{l}\text { I was thinking } \\
\text { about what I was } \\
\text { going to do when } \\
\text { the study was } \\
\text { done. }\end{array}$ & 1 & 2 & 3 & 4 & 5 & 6 & 7 \\
\hline $\begin{array}{l}\text { I thought the task } \\
\text { was a waste of my } \\
\text { time. }\end{array}$ & 1 & 2 & 3 & 4 & 5 & 6 & 7 \\
\hline $\begin{array}{l}\text { I was thinking that } \\
\text { the task was going } \\
\text { on for a long time. }\end{array}$ & 1 & 2 & 3 & 4 & 5 & 6 & 7 \\
\hline
\end{tabular}


At one point during this study, we asked you view photographs of a positive event that you provided.

How happy were you about this event when it first happened?

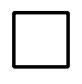

Not at all
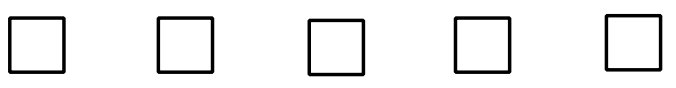

Somewhat happy

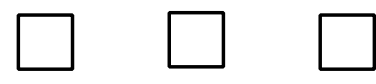

Extremely

happy

What emotions would you use to describe your feelings about the event?

How long ago did this event happen?

How much did you feel like this event was in your control?

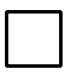

Not

at all
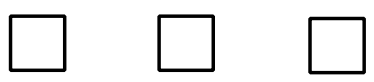

Somewhat

in my control
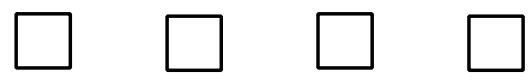

Extremely

in my control

How spontaneous was this event? (Meaning you did not know it was going to happen in advance)

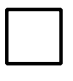

Not

at all

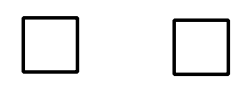

Somewhat

spontaneous
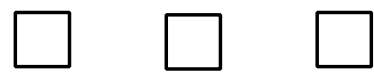

Extremely

spontaneous

Did you ever have a negative relationship with the people in this photograph? (Skip this question, if there are no other people in these photographs)?

$\square$ Yes

$\square$ No 
If there are other people in your photographs, please describe your relationship with these people. You can choose more than one category. (Skip this question, if there are no other people in these Photographs).

Friendship

Romantic (Please specify): Past Present

Roommate

Work/colleague/classmate

Work superior (ex. Boss)

Your child

Your parent

Other (Please specify):

Is there anything else you want to add about the event?

When you were viewing the photographs, you were also completing math problems. How much were you distracted by the photos when completing the math problems? (Please mark anywhere along the line)

\begin{tabular}{lllllllllll}
\hline $0 \%$ & $10 \%$ & $20 \%$ & $30 \%$ & $40 \%$ & $50 \%$ & $60 \%$ & $70 \%$ & $80 \%$ & $90 \%$ & $100 \%$
\end{tabular}

Not at all

Very distracted

When you were completing the math problems, you also held chopsticks in your mouth. How difficult was it to hold the chopsticks in your mouth? (Please mark anywhere along the line)

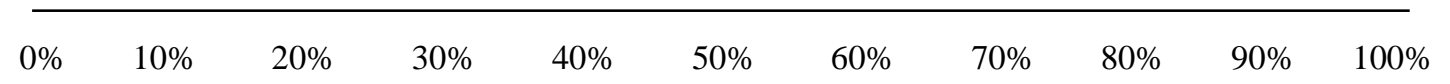

Not at all

Very difficult 
How tired was your mouth as you held the chopsticks in your mouth? (Please mark anywhere along the line)

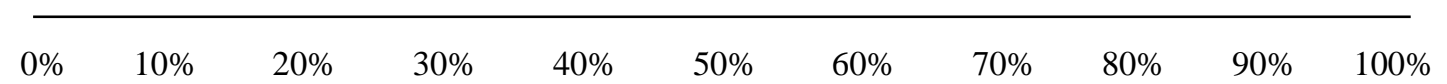

Not at all

Very tired

How uncomfortable was it to hold the chopsticks in your mouth? (Please mark anywhere along the line)

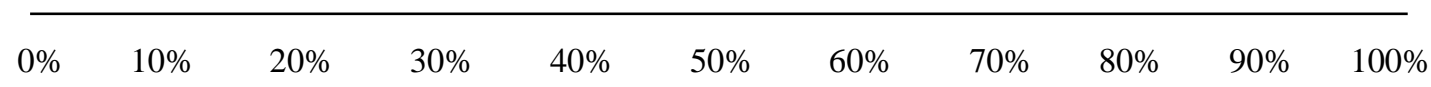

Not at all

Very uncomfortable

Do you know why we asked you to hold the chopsticks in your mouth?

$\square$ Yes $\quad \square$ No

If you answered yes to the above question, please explain why you think we asked you to hold chopsticks in your mouth? (Skip this question if you answered no to the above question).

When you finished the math problems, you sat while more physiological measures (heart rate and blood pressure) were obtained. Please answer these following questions about what you were thinking during the final rest period following the math problems.

1. What did you think about most of the time? 
How many minutes of the 10 -min rest period would you estimate that you thought about what you reported above?

2. If you thought about more than one thing during the rest period, what did you think about next most?

How many minutes of the 10 -min rest period would you estimate that you thought about what you reported above?

3. If you thought about more than two things during the rest period, what did you think about next most?

How many minutes of the 10 -min rest period would you estimate that you thought about what you reported above? 


\section{Appendix K}

\section{Post-Experiment Questionnaire-B}

Please indicate your agreement with the following statements about the math task (where you were completing math problems).

\begin{tabular}{|c|c|c|c|c|c|c|c|}
\hline & $\begin{array}{l}\text { Strongly } \\
\text { Disagree }\end{array}$ & & & & & & $\begin{array}{l}\text { Strongly } \\
\text { Agree }\end{array}$ \\
\hline $\begin{array}{l}\text { I was impatient for } \\
\text { the task to end so I } \\
\text { could finish the } \\
\text { study. }\end{array}$ & 1 & 2 & 3 & 4 & 5 & 6 & 7 \\
\hline $\begin{array}{l}\text { My thoughts were } \\
\text { completely } \\
\text { absorbed by the } \\
\text { task. }\end{array}$ & 1 & 2 & 3 & 4 & 5 & 6 & 7 \\
\hline $\begin{array}{l}\text { I felt the task was a } \\
\text { relaxing break. }\end{array}$ & 1 & 2 & 3 & 4 & 5 & 6 & 7 \\
\hline $\begin{array}{l}\text { The task inspired } \\
\text { me to think } \\
\text { pleasant thoughts. }\end{array}$ & 1 & 2 & 3 & 4 & 5 & 6 & 7 \\
\hline $\begin{array}{l}\text { I was enjoying the } \\
\text { task and I did not } \\
\text { want it to end. }\end{array}$ & 1 & 2 & 3 & 4 & 5 & 6 & 7 \\
\hline $\begin{array}{l}\text { I was thinking } \\
\text { about what I was } \\
\text { going to do when } \\
\text { the study was } \\
\text { done. }\end{array}$ & 1 & 2 & 3 & 4 & 5 & 6 & 7 \\
\hline $\begin{array}{l}\text { I thought the task } \\
\text { was a waste of my } \\
\text { time. }\end{array}$ & 1 & 2 & 3 & 4 & 5 & 6 & 7 \\
\hline $\begin{array}{l}\text { I was thinking that } \\
\text { the task was going } \\
\text { on for a long time. }\end{array}$ & 1 & 2 & 3 & 4 & 5 & 6 & 7 \\
\hline
\end{tabular}


At one point during this study, we asked you view photographs that you had not seen before.

How neutral (not good or bad) were these photographs?

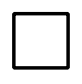

Not at all
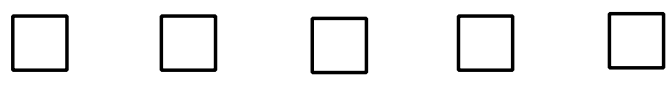

Somewhat neutral

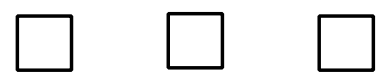

Extremely neutral

Were there other emotions that you experienced when viewing these photographs?

When you were viewing the photographs, you were also completing math problems. How much were you distracted by the photos when completing the math problems? (Please mark anywhere along the line)

\begin{tabular}{ccccccccccc}
$0 \%$ & $10 \%$ & $20 \%$ & $30 \%$ & $40 \%$ & $50 \%$ & $60 \%$ & $70 \%$ & $80 \%$ & $90 \%$ & $100 \%$ \\
\hline Not at all & & & & & Very distracted
\end{tabular}

When you were completing the math problems, you also held chopsticks in your mouth. How difficult was it to hold the chopsticks in your mouth? (Please mark anywhere along the line)

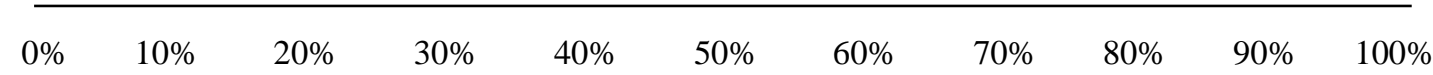

Not at all

Very difficult

How tired was your mouth as you held the chopsticks in your mouth? (Please mark anywhere along the line)

\begin{tabular}{lllllllllll}
\hline $0 \%$ & $10 \%$ & $20 \%$ & $30 \%$ & $40 \%$ & $50 \%$ & $60 \%$ & $70 \%$ & $80 \%$ & $90 \%$ & $100 \%$
\end{tabular}

Not at all

Very tired 
How uncomfortable was it to hold the chopsticks in your mouth? (Please mark anywhere along the line)

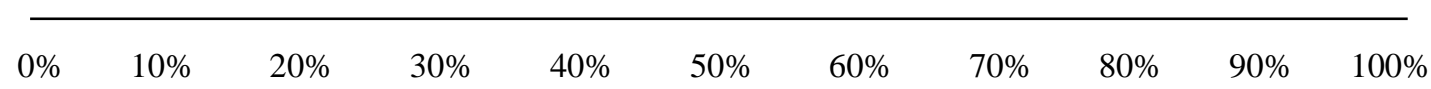

Not at all

Very uncomfortable

Do you know why we asked you to hold the chopsticks in your mouth?

$\square$ Yes $\quad \square$ No

If you answered yes to the above question, please explain why you think we asked you to hold chopsticks in your mouth? (Skip this question if you answered no to the above question).

When you finished the math problems, you sat while more physiological measures (heart rate and blood pressure) were obtained. Please answer these following questions about what you were thinking during the final rest period following the math problems.

1. What did you think about most of the time?

How many minutes of the 10 -min rest period would you estimate that you thought about what you reported above?

2. If you thought about more than one thing during the rest period, what did you think about next most? 
How many minutes of the 10 -min rest period would you estimate that you thought about what you reported above?

3. If you thought about more than two things during the rest period, what did you think about next most?

How many minutes of the 10 -min rest period would you estimate that you thought about what you reported above? 